\title{
On the Superior Activity of In(I) versus In(III) Cations Towards ortho-C-Alkylation of Anilines and Intramolecular Hydroamination of Alkenes
}

Zhilong $\mathrm{Li}^{+}$Shengwen Yang, ${ }^{+, \neq}$Guillaume Thiery, ${ }^{\dagger}$ Vincent Gandon, ${ }^{*,+, \neq}$ and Christophe Bour*,+

†Institut de Chimie Moléculaire et des Matériaux d'Orsay (ICMMO), CNRS UMR 8182, Université Paris-Saclay, Bâtiment 420, 91405 Orsay cedex, France.

‡Laboratoire de Chimie Moléculaire (LCM), CNRS UMR 9168, Ecole Polytechnique, Institut Polytechnique de Paris, route de Saclay, 91128 Palaiseau cedex, France.

christophe.bour@universite-paris-saclay.fr

vincent.gandon@universite-paris-saclay.fr

\section{Supporting Information}

\section{CONTENTS}

1. Optimization of solvents and temperature for the intramolecular hydroamination of

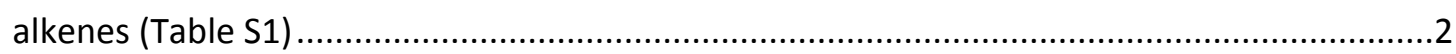

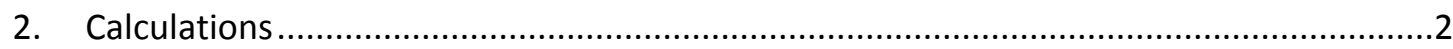

2.1. Mechanism of the $\operatorname{In}(\mathrm{I})$-catalyzed hydroamination reaction with two ligands ....2

2.2. Mechanism of the $\ln (\mathrm{I})$-catalyzed hydroamination reaction with one ligand.......3

2.3. Isomerization reaction of the $\ln (\mathrm{I})$-catalyzed hydroamination without ligand ....4

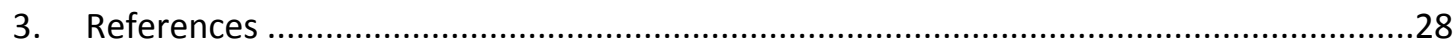

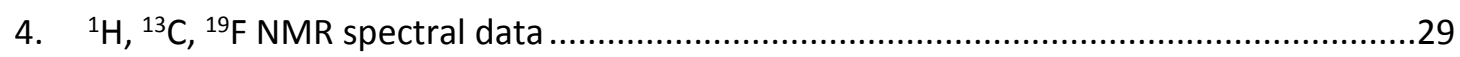




\section{Optimization of solvents and temperature for the intramolecular}

\section{hydroamination of alkenes (Table S1)}

General procedure for the intramolecular hydroamination of alkenes (Table S1). In a glove box, $\left[\ln (\mathrm{PhF})_{2}\right]\left[\mathrm{Al}\left(\mathrm{OC}\left(\mathrm{CF}_{3}\right)_{3}\right)_{4}\right]^{20 \mathrm{a}, \mathrm{b}}(0.05$ equiv, $0.02 \mathrm{mmol})$ was added into anhydrous degassed solvent $(0.5 \mathrm{~mL})$ in a reaction tube. Then aminoalkenes 5 a $(47.5 \mathrm{mg}, 0.2 \mathrm{mmol})$ were added in this solution. The reaction tube was sealed with a screw cap and stirred outside of the glove box at the corresponding temperature for $\mathbf{1 2} \mathbf{h}$. The reaction mixture was diluted with $\mathrm{Et}_{2} \mathrm{O}$, filtered through a pad of of aluminum oxide (thoroughly rinsed with $\mathrm{Et}_{2} \mathrm{O}$ ) and concentrated under reduced pressure by rotary evaporation. Purification by FC over neutral $\mathrm{Al}_{2} \mathrm{O}_{3}$ (cyclohexane/EtOAc $=95 / 5$ ) afforded product $\mathbf{6 a}$.

Table S1. Solvents screening for the intramolecular hydroamination of alkenes

\begin{tabular}{lllll}
\hline Entry & Catalyst & Solvent & Temp. & Yield $^{a}$ \\
\hline 1 & {$\left[\ln (\mathrm{PhF})_{2}\right]\left[\mathrm{Al}\left(\mathrm{OC}\left(\mathrm{CF}_{3}\right)_{3}\right)_{4}\right]$} & Toluene $(0.2 \mathrm{M})$ & $110{ }^{\circ} \mathrm{C}$ & 56 \\
2 & {$\left[\ln (\mathrm{PhF})_{2}\right]\left[\mathrm{Al}\left(\mathrm{OC}\left(\mathrm{CF}_{3}\right)_{3}\right)_{4}\right]$} & $\mathrm{PhF}(0.2 \mathrm{M})$ & $85^{\circ} \mathrm{C}$ & 71 \\
3 & {$\left[\ln (\mathrm{PhF})_{2}\right]\left[\mathrm{Al}\left(\mathrm{OC}\left(\mathrm{CF}_{3}\right)_{3}\right)_{4}\right]$} & $\mathrm{DCE}(0.2 \mathrm{M})$ & $80^{\circ} \mathrm{C}$ & 24 \\
4 & {$\left[\ln (\mathrm{PhF})_{2}\right]\left[\mathrm{Al}\left(\mathrm{OC}\left(\mathrm{CF}_{3}\right)_{3}\right)_{4}\right]$} & $\mathrm{THF}(0.2 \mathrm{M})$ & $60^{\circ} \mathrm{C}$ & 47 \\
5 & {$\left[\ln (\mathrm{PhF})_{2}\right]\left[\mathrm{Al}\left(\mathrm{OC}\left(\mathrm{CF}_{3}\right)_{3}\right)_{4}\right]$} & $\mathrm{PhF}(0.2 \mathrm{M})$ & $110^{\circ} \mathrm{C}$ & 78 \\
6 & {$\left[\ln (\mathrm{PhF})_{2}\right]\left[\mathrm{Al}\left(\mathrm{OC}\left(\mathrm{CF}_{3}\right)_{3}\right)_{4}\right]$} & $\mathrm{PhF}(0.4 \mathrm{M})$ & $110^{\circ} \mathrm{C}$ & $89\left(99^{b}\right)$ \\
\hline
\end{tabular}

${ }^{a}$ Isolated yield by chromatography column on $\mathrm{Al}_{2} \mathrm{O}_{3}(\mathrm{Cyclohexane} / \mathrm{EtOAC}=95 / 5)$.

${ }^{b}$ Determined by ${ }^{1} \mathrm{H}$ NMR spectroscopy using ferrocene ( 0.5 equiv) as the internal standard.

\section{Calculations}

All calculations were carried out using the M06-2X functional ${ }^{1}$ implemented in the Gaussian 09 program package. ${ }^{2}$ For geometry optimizations, the LANL2DZ3,4 pseudopotential with effective core potential (ECP) was used for $\mathrm{In}$, and the $6-31 \mathrm{G}(\mathrm{d}, \mathrm{p})^{5}$ basis set was used for all other atoms. The stationary points were confirmed as minima (no imaginary frequencies) or transition states (only one imaginary frequency) by analytical frequency calculations at the same theory level as the geometry optimizations. On the basis of these optimized geometries, single-point calculations were carried out with the Def2-TZVP6 basis set for In and the $6-311+G(2 d, p)$ basis set for all other elements. The reported energies are Gibbs free energies, which include solvation free energies. The latter are calculated as single-point corrections on the optimized structures using the SMD method ${ }^{7}$ with the parameters for fluorobenzene. Intrinsic Reaction Coordinate (IRC) ${ }^{8,9}$ calculations were performed to ensure that the saddle points located were transition states connecting the reactants and the products. Molecular structures were visualized using CYLview. ${ }^{10}$ 


\subsection{Mechanism of the $\ln (\mathrm{I})$-catalyzed hydroamination reaction with two ligands}

Considering that $\ln (\mathrm{PhF})_{2}{ }^{+}$may keep the PhF ligands, we evaluated the free energy profile of the proton migration step without releasing the ligand based on the transition states $9^{\text {zero-ts }}$

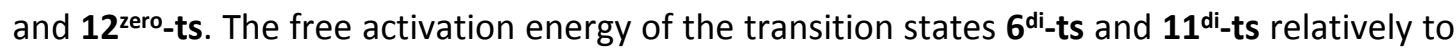
the reference point are $47.6 \mathrm{kcal} / \mathrm{mol}$ and $55.5 \mathrm{kcal} / \mathrm{mol}$, respectively, which is not realistic at $383 \mathrm{~K}$. Therefore, we did not proceed further with this hypothesis.

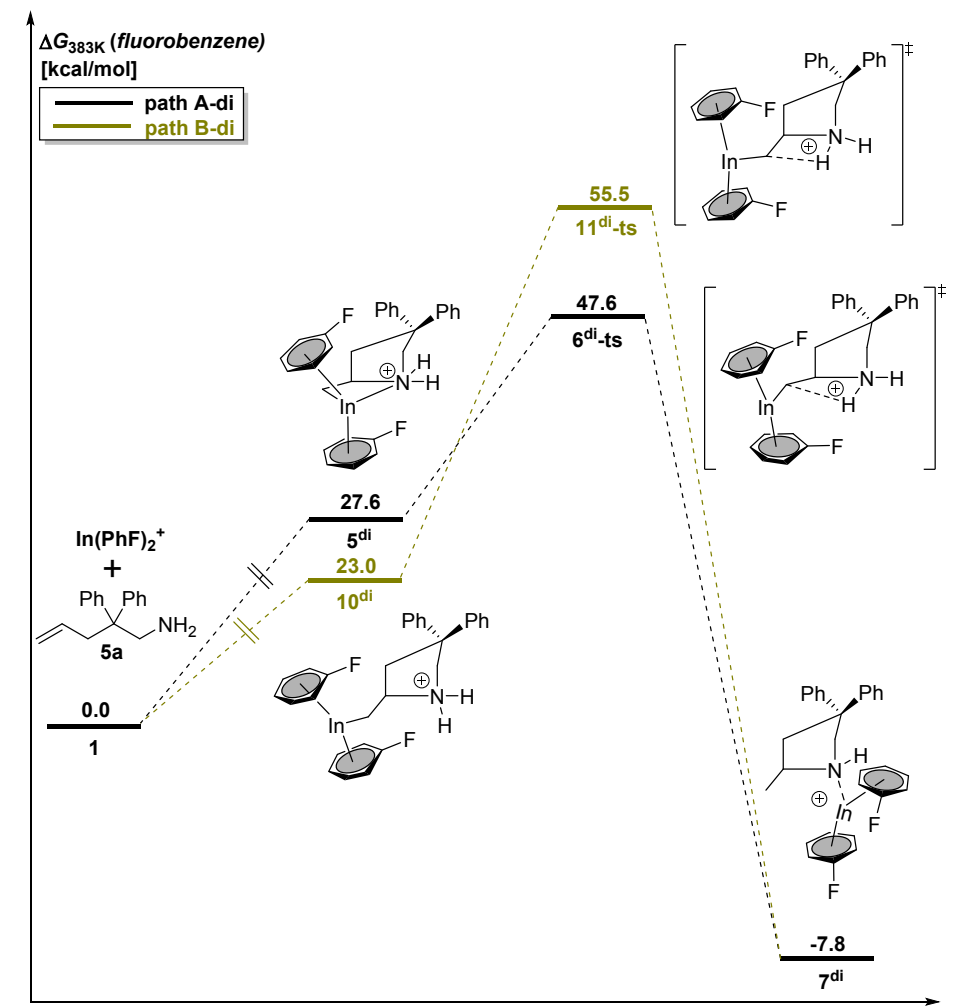

Figure S1. Calculated energy profile of the two-ligand catalyst in the proton migration step. Solvent-corrected free energies are given in $\mathrm{kcal} / \mathrm{mol}$.

\subsection{Mechanism of the In(I)-catalyzed hydroamination reaction with one ligand}

Releasing one PhF ligand was also calculated (Figures S2a and S2b). However, the energy

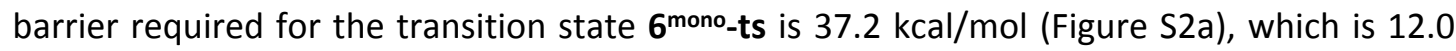

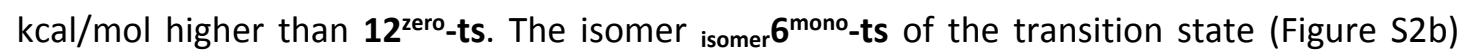
has a similar energy barrier $(37.1 \mathrm{kcal} / \mathrm{mol})$. Therefore, we also ruled out the possibility of this mechanism 


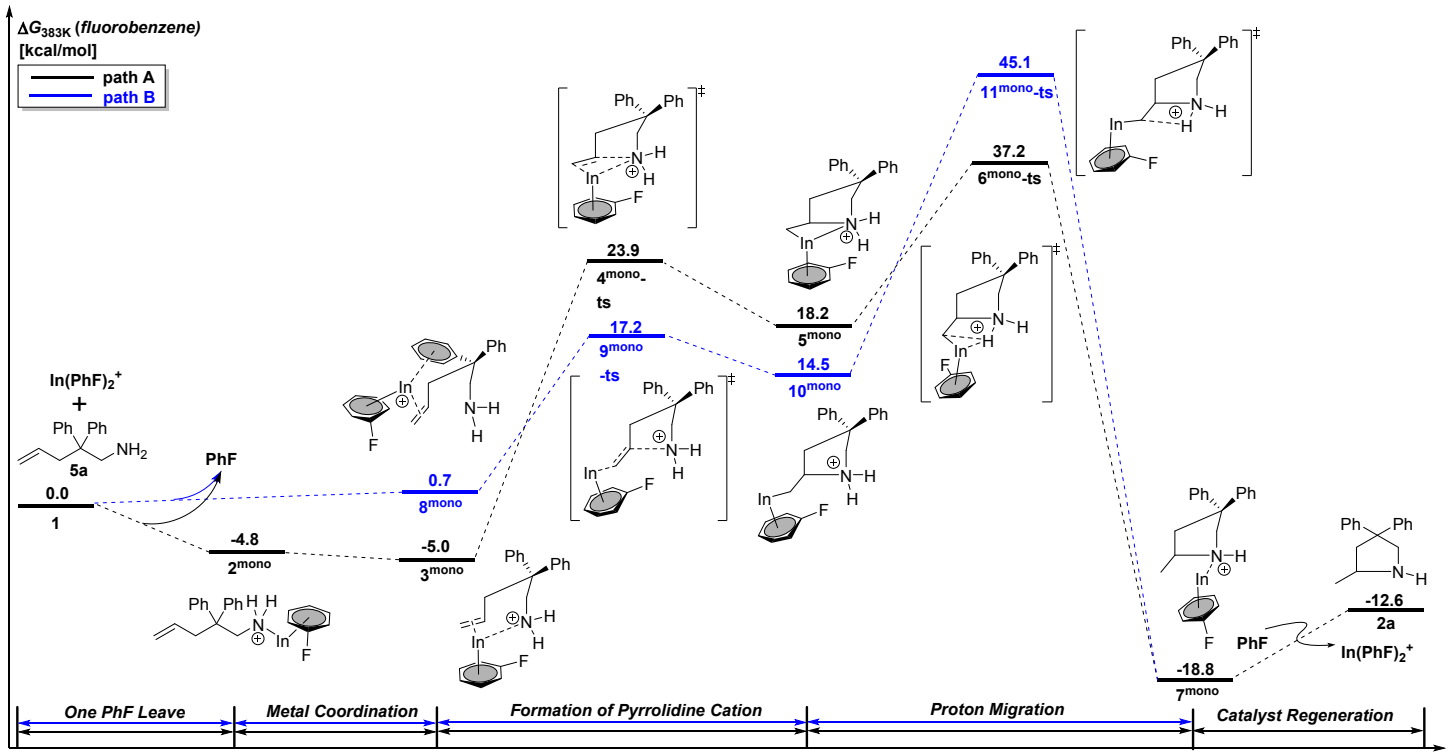

Figure S2a. Calculated free energy profile for the $\ln (\mathrm{I})$-catalyzed hydroamination reaction with one PhF. Solvent-corrected free energies are given in $\mathrm{kcal} / \mathrm{mol}$.

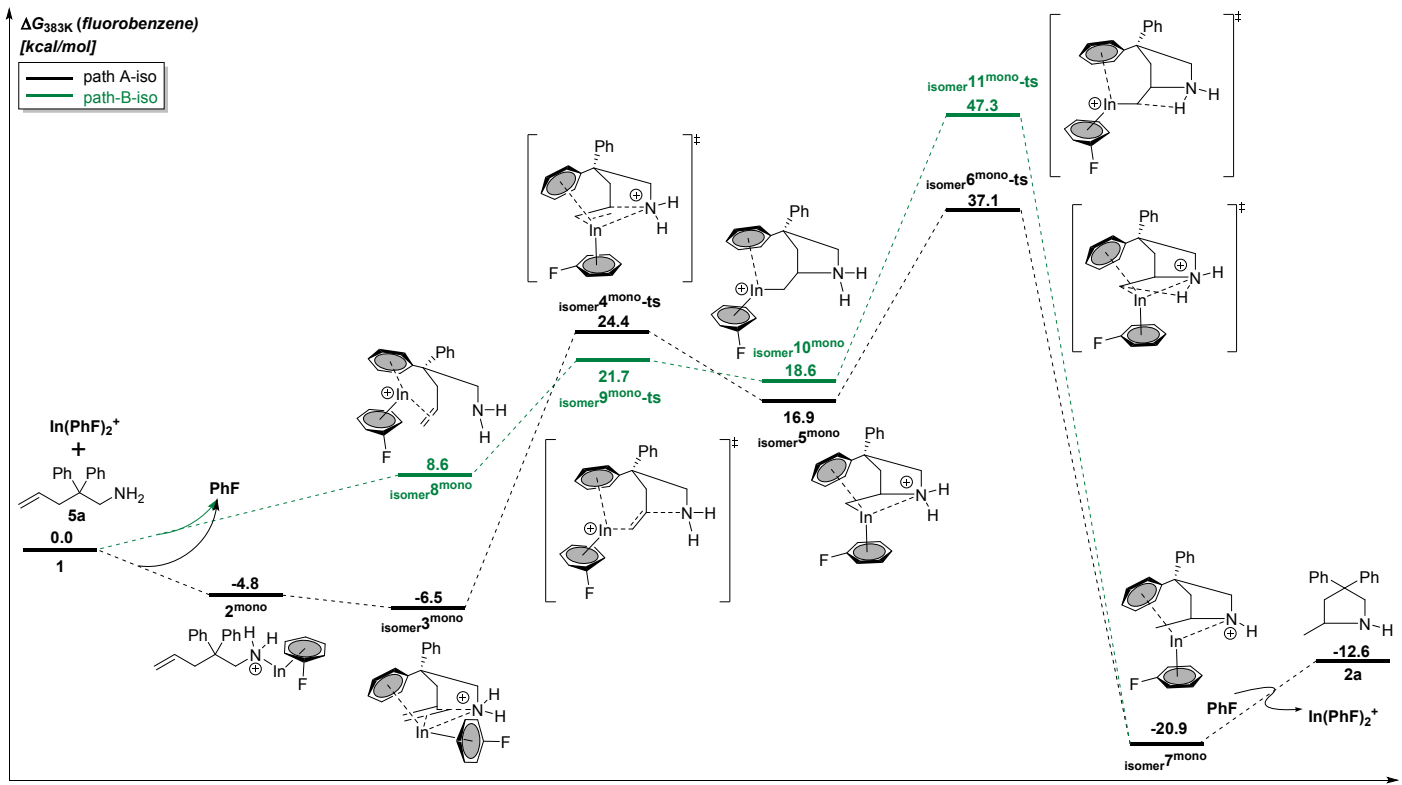

Figure S2b. Calculated free energy profile of the isomerization reaction of the $\ln (\mathrm{I})$-catalyzed hydroamination reaction with one PhF. Solvent-corrected free energies are given in $\mathrm{kcal} / \mathrm{mol}$.

\subsection{Isomerization reaction of the $\ln (\mathrm{I})$-catalyzed hydroamination without ligand}

We have also evaluated the isomerization mechanism in the case of the release of two PhF ligands. The free activation energy of isomer 6 $^{\text {zero-ts }}(28.5 \mathrm{kcal} / \mathrm{mol})$ is higher than the free activation energy of $6^{\text {zero-ts }}(25.2 \mathrm{kcal} / \mathrm{mol})$, indicating that it is a less favorable pathway. 


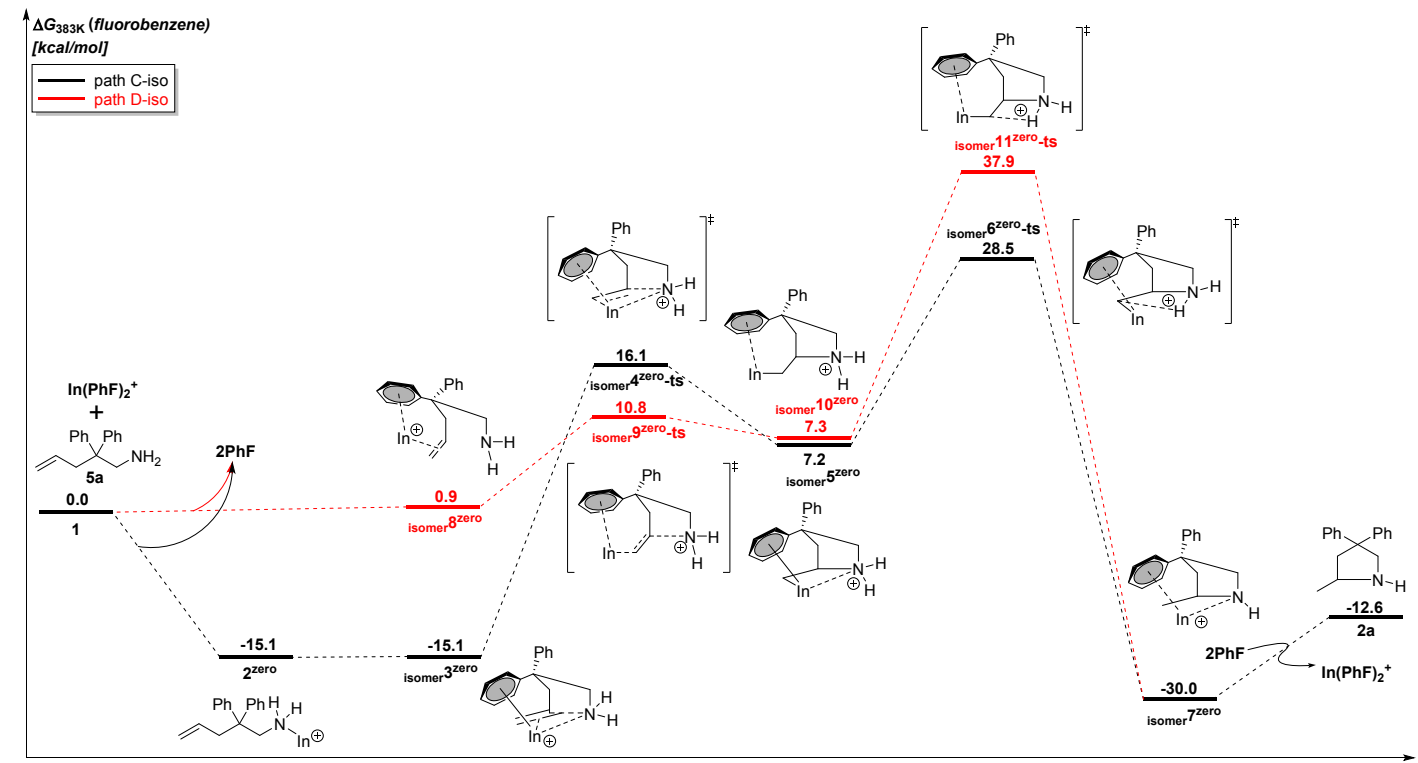

Figure S3. Calculated free energy profile of the isomerization reaction of the $\ln (\mathrm{I})$-catalyzed hydroamination reaction without ligand. Solvent-corrected free energies are given in $\mathrm{kcal} / \mathrm{mol}$.

Table S2. Geometries (energies (Hartree) and coordinates of the computed species $(x, y, z)$.

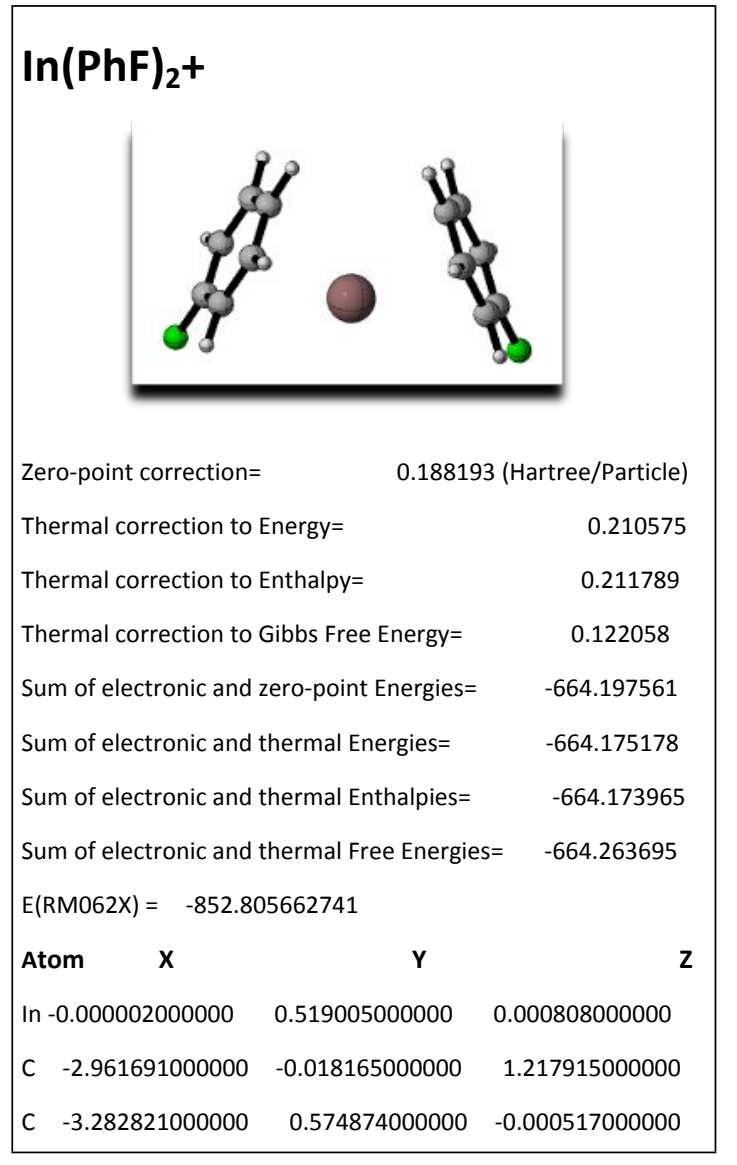

\begin{tabular}{|lrrr|}
\hline C & -2.960699000000 & -0.017991000000 & -1.218770000000 \\
C & -2.315487000000 & -1.256854000000 & -1.210573000000 \\
C & -1.987760000000 & -1.877477000000 & -0.000162000000 \\
C & -2.316468000000 & -1.257023000000 & 1.210067000000 \\
H & -3.245214000000 & 0.476668000000 & 2.140463000000 \\
H & -3.243475000000 & 0.476969000000 & -2.141478000000 \\
H & -2.086121000000 & -1.744542000000 & -2.152589000000 \\
H & -1.512055000000 & -2.852484000000 & -0.000032000000 \\
H & -2.087869000000 & -1.744834000000 & 2.152206000000 \\
F & -3.892993000000 & 1.754260000000 & -0.000679000000 \\
C & 2.960699000000 & -0.017986000000 & -1.218771000000 \\
C & 3.282819000000 & 0.574880000000 & -0.000516000000 \\
C & 2.961694000000 & -0.018162000000 & 1.217915000000 \\
C & 2.316478000000 & -1.257024000000 & 1.210066000000 \\
C & 1.987772000000 & -1.877479000000 & -0.000163000000 \\
C & 2.315494000000 & -1.256853000000 & -1.210574000000 \\
H & 3.243472000000 & 0.476976000000 & -2.141479000000 \\
H & 3.245212000000 & 0.476673000000 & 2.140463000000 \\
H & 2.087884000000 & -1.744839000000 & 2.152204000000 \\
H & 1.512074000000 & -2.852489000000 & -0.000033000000 \\
H & 2.086131000000 & -1.744542000000 & -2.152590000000 \\
\hline & & & \\
\hline
\end{tabular}


F $\quad 3.892983000000 \quad 1.754270000000 \quad-0.000679000000$

$5 a$

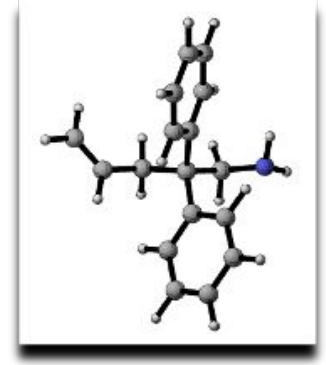

Zero-point correction=

0.320444 (Hartree/Particle)

Thermal correction to Energy=

0.346517

Thermal correction to Enthalpy=

0.347731

Thermal correction to Gibbs Free Energy=

0.258080

Sum of electronic and zero-point Energies=

$-713.345008$

Sum of electronic and thermal Energies=

$-713.318935$

Sum of electronic and thermal Enthalpies=

$-713.317721$

Sum of electronic and thermal Free Energies=

$-713.407372$

$E(R M 062 X)=-713.864198534$

Atom

$$
\mathbf{X}
$$

C -4.726119000000

C $-3.490628000000 \quad 1.440027000000$

C -2.308314000000

C $-1.759642000000 \quad 0.142917000000$

C $-0.447742000000 \quad-0.642299000000$

N $\quad 0.182851000000-1.199194000000$

C $-1.345125000000 \quad 1.371279000000$

C $-0.866351000000 \quad 2.526570000000$

C $-0.368769000000 \quad 3.597725000000$

C $-0.321709000000 \quad 3.533980000000-1.728682000000$

C $-0.781726000000 \quad 2.389227000000 \quad-2.373342000000$

C $-1.289439000000 \quad 1.322312000000-1.631832000000$

C $-2.811757000000 \quad-0.723632000000 \quad-0.103571000000$

C $-2.823762000000 \quad-2.116622000000 \quad 0.034774000000$

C $-3.834483000000 \quad-2.891589000000 \quad-0.531436000000$

C $-4.863967000000 \quad-2.283819000000 \quad-1.240453000000$

C $-4.879987000000 \quad-0.896968000000 \quad-1.366627000000$

C -3.868117000000 $-0.127413000000 \quad-0.802995000000$

H -5.559186000000 $1.747684000000 \quad 2.278023000000$

H -4.945680000000 $0.024600000000 \quad 2.568954000000$

H -3.305788000000 $2.475357000000 \quad 1.711220000000$

H-1.495213000000 $0.946259000000 \quad 2.601787000000$

$\begin{array}{lll}H-2.591350000000 & -0.424572000000 & 2.492817000000\end{array}$

\begin{tabular}{|c|c|c|c|}
\hline $\mathrm{H}$ & 0.265017000000 & 0.044842000000 & 1.314571000000 \\
\hline \multicolumn{2}{|c|}{ H - 0.616738000000} & -1.466219000000 & 1.545877000000 \\
\hline \multicolumn{2}{|c|}{ H - 0.449932000000} & -1.897957000000 & -0.775532000000 \\
\hline \multicolumn{2}{|r|}{ H - 0.874122000000} & 2.601869000000 & 1.481980000000 \\
\hline \multicolumn{2}{|r|}{ H - 0.016170000000} & 4.484248000000 & 0.179006000000 \\
\hline $\mathrm{H}$ & 0.061496000000 & 4.370779000000 & -2.302855000000 \\
\hline \multicolumn{2}{|c|}{ H - 0.763813000000} & 2.328269000000 & -3.456764000000 \\
\hline \multicolumn{2}{|c|}{ H -1.681925000000 } & 0.453562000000 & -2.153895000000 \\
\hline \multicolumn{2}{|c|}{ H - 2.058285000000} & -2.624511000000 & 0.617299000000 \\
\hline \multicolumn{2}{|c|}{ H -3.818038000000} & -3.969436000000 & -0.408011000000 \\
\hline \multicolumn{2}{|c|}{ H -5.652685000000} & -2.883128000000 & -1.682345000000 \\
\hline \multicolumn{2}{|c|}{$H-5.687850000000$} & -0.408245000000 & -1.901042000000 \\
\hline \multicolumn{2}{|c|}{ H -3.901499000000 } & 0.953194000000 & -0.898912000000 \\
\hline \multicolumn{2}{|c|}{ H $\quad 0.218669000000$} & -0.449757000000 & -1.075165000000 \\
\hline \multicolumn{4}{|c|}{$2^{\text {mono }}$} \\
\hline \multicolumn{3}{|c|}{ Zero-point correction= } & 362 (Hartree/Particle) \\
\hline \multicolumn{3}{|c|}{ Thermal correction to Energy= } & 0.456631 \\
\hline \multicolumn{3}{|c|}{ Thermal correction to Enthalpy= } & 0.457844 \\
\hline \multicolumn{3}{|c|}{ Thermal correction to Gibbs Free Energy= } & 0.334399 \\
\hline \multicolumn{3}{|c|}{ Sum of electronic and zero-point Energies= } & -1046.326536 \\
\hline \multicolumn{3}{|c|}{ Sum of electronic and thermal Energies= } & -1046.287267 \\
\hline \multicolumn{3}{|c|}{ Sum of electronic and thermal Enthalpies= } & -1046.286054 \\
\hline \multicolumn{3}{|c|}{ Sum of electronic and thermal Free Energies= } & ies $=\quad-1046.409499$ \\
\hline \multicolumn{4}{|c|}{$E(R M 062 X)=-1235.24564234$} \\
\hline \multicolumn{2}{|c|}{ Atom } & $\mathbf{Y}$ & $\mathbf{Z}$ \\
\hline C & -4.726119000000 & 1.053422000000 & 2.294448000000 \\
\hline C & -3.490628000000 & 1.440027000000 & 1.992334000000 \\
\hline C & -2.308314000000 & 0.513888000000 & 2.003226000000 \\
\hline C & -1.759642000000 & 0.142917000000 & 0.591940000000 \\
\hline C & -0.447742000000 & -0.642299000000 & 0.844674000000 \\
\hline $\mathrm{N}$ & 0.182851000000 & $0-1.199194000000$ & $0-0.378159000000$ \\
\hline C & -1.345125000000 & 1.371279000000 & -0.230658000000 \\
\hline C & -0.866351000000 & 2.526570000000 & 0.399787000000 \\
\hline C & -0.368769000000 & 3.597725000000 & -0.337969000000 \\
\hline C & -0.321709000000 & 3.533980000000 & -1.728682000000 \\
\hline C & -0.781726000000 & 2.389227000000 & -2.373342000000 \\
\hline
\end{tabular}




\begin{tabular}{|c|c|c|c|}
\hline C & -1.289439000000 & 1.322312000000 & -1.631832000000 \\
\hline C & -2.811757000000 & -0.723632000000 & -0.103571000000 \\
\hline C & -2.823762000000 & -2.116622000000 & 0.034774000000 \\
\hline C & -3.834483000000 & -2.891589000000 & -0.531436000000 \\
\hline C & -4.863967000000 & -2.283819000000 & -1.240453000000 \\
\hline C & -4.879987000000 & -0.896968000000 & -1.366627000000 \\
\hline C & -3.868117000000 & -0.127413000000 & -0.802995000000 \\
\hline H & -5.559186000000 & 1.747684000000 & 2.278023000000 \\
\hline $\mathrm{H}$ & -4.945680000000 & 0.024600000000 & 2.568954000000 \\
\hline $\mathrm{H}$ & -3.305788000000 & 2.475357000000 & 1.711220000000 \\
\hline $\mathrm{H}$ & -1.495213000000 & 0.946259000000 & 2.601787000000 \\
\hline $\mathrm{H}$ & -2.591350000000 & -0.424572000000 & 2.492817000000 \\
\hline $\mathrm{H}$ & 0.265017000000 & 0.044842000000 & 1.314571000000 \\
\hline $\mathrm{H}$ & -0.616738000000 & -1.466219000000 & 1.545877000000 \\
\hline $\mathrm{H}$ & -0.449932000000 & -1.897957000000 & -0.775532000000 \\
\hline H & -0.874122000000 & 2.601869000000 & 1.481980000000 \\
\hline $\mathrm{H}$ & -0.016170000000 & 4.484248000000 & 0.179006000000 \\
\hline $\mathrm{H}$ & 0.061496000000 & 4.370779000000 & -2.302855000000 \\
\hline $\mathrm{H}$ & -0.763813000000 & 2.328269000000 & -3.456764000000 \\
\hline $\mathrm{H}$ & -1.681925000000 & 0.453562000000 & -2.153895000000 \\
\hline $\mathrm{H}$ & -2.058285000000 & -2.624511000000 & 0.617299000000 \\
\hline $\mathrm{H}$ & -3.818038000000 & -3.969436000000 & -0.408011000000 \\
\hline H & -5.652685000000 & -2.883128000000 & -1.682345000000 \\
\hline $\mathrm{H}$ & -5.687850000000 & -0.408245000000 & -1.901042000000 \\
\hline $\mathrm{H}$ & -3.901499000000 & 0.953194000000 & -0.898912000000 \\
\hline $\mathrm{H}$ & 0.218669000000 & -0.449757000000 & -1.075165000000 \\
\hline In & 2.413686000000 & -1.944075000000 & 0.114374000000 \\
\hline C & 2.362029000000 & 1.214388000000 & -0.545891000000 \\
\hline C & 2.745829000000 & 1.270170000000 & 0.790388000000 \\
\hline C & 4.008450000000 & 0.875016000000 & 1.220697000000 \\
\hline C & 4.925800000000 & 0.427547000000 & 0.270036000000 \\
\hline C & 4.569724000000 & 0.354638000000 & -1.077648000000 \\
\hline C & 3.290544000000 & 0.747467000000 & -1.481372000000 \\
\hline $\mathrm{H}$ & 1.381083000000 & 1.578593000000 & -0.839695000000 \\
\hline $\mathrm{H}$ & 4.260406000000 & 0.949314000000 & 2.272827000000 \\
\hline H & 5.920697000000 & 0.133587000000 & 0.586422000000 \\
\hline $\mathrm{H}$ & 5.290017000000 & 0.011853000000 & -1.812275000000 \\
\hline $\mathrm{H}$ & 3.018973000000 & 0.723159000000 & -2.532292000000 \\
\hline$F$ & 1.862547000000 & 1.706811000000 & 1.699088000000 \\
\hline \multicolumn{4}{|c|}{$3^{\text {mono }}$} \\
\hline
\end{tabular}

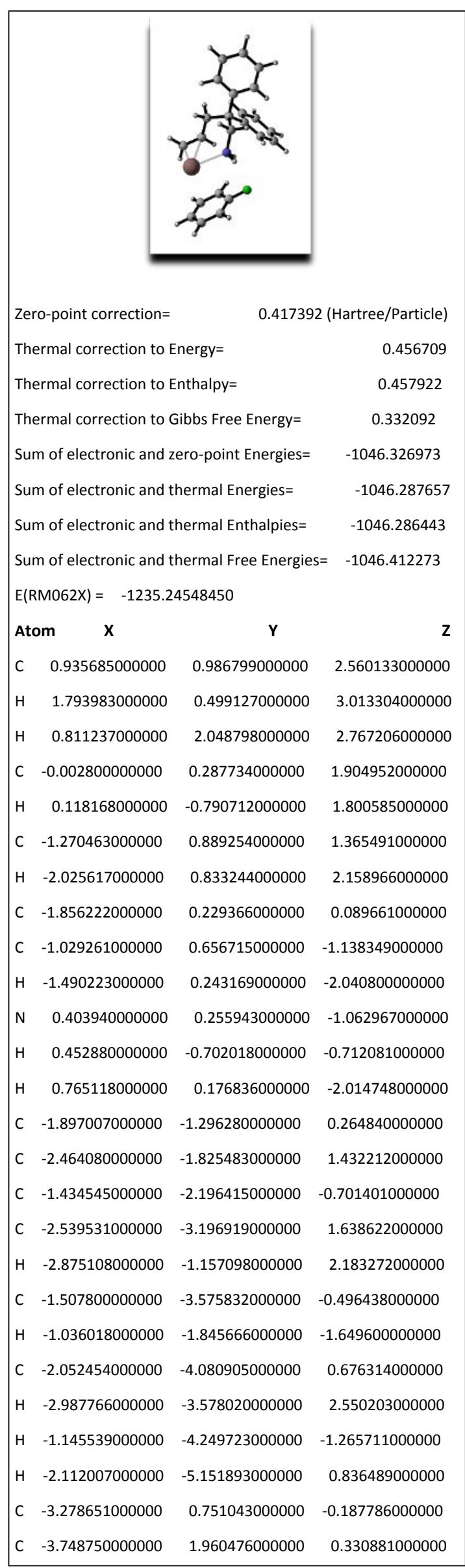




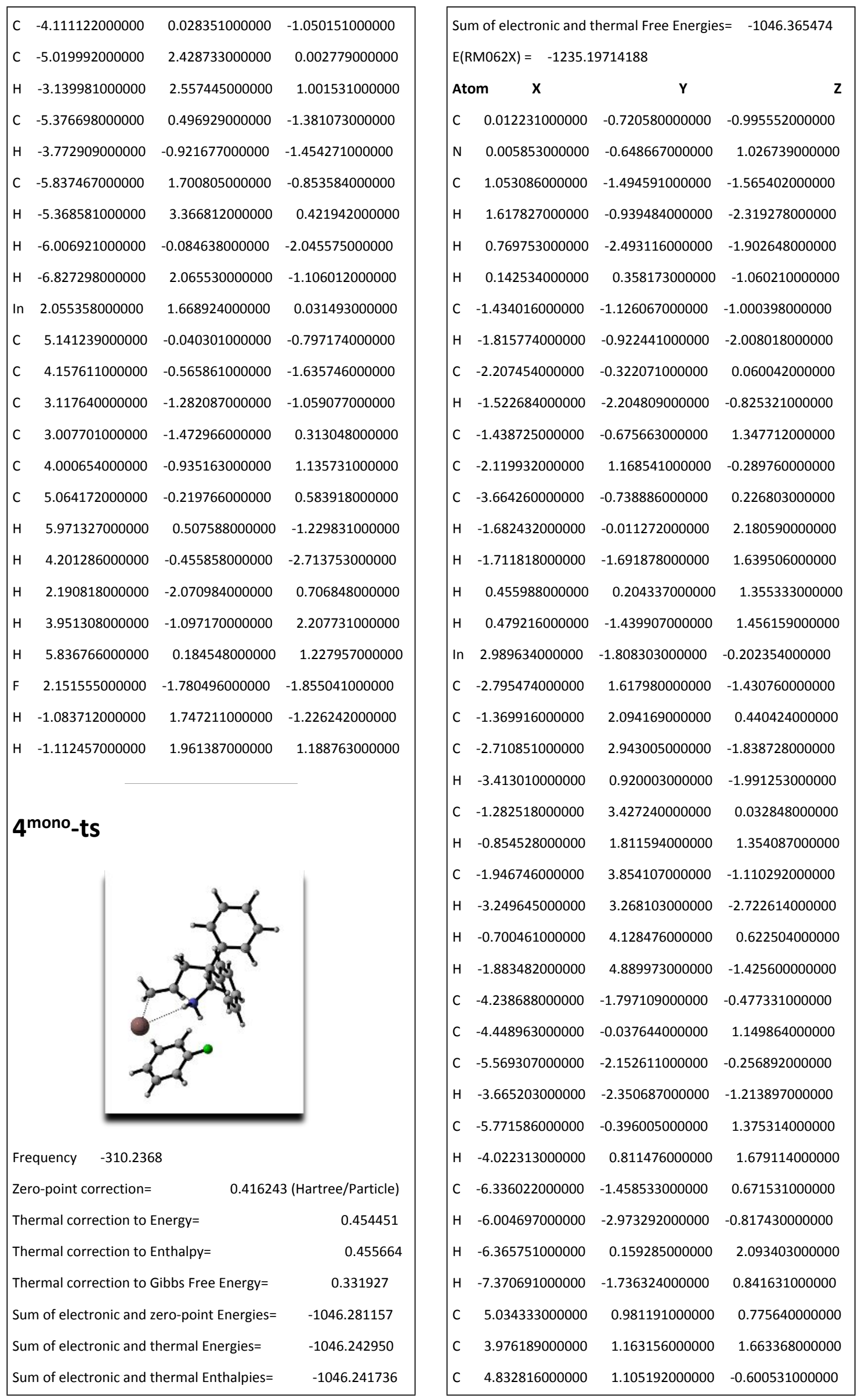




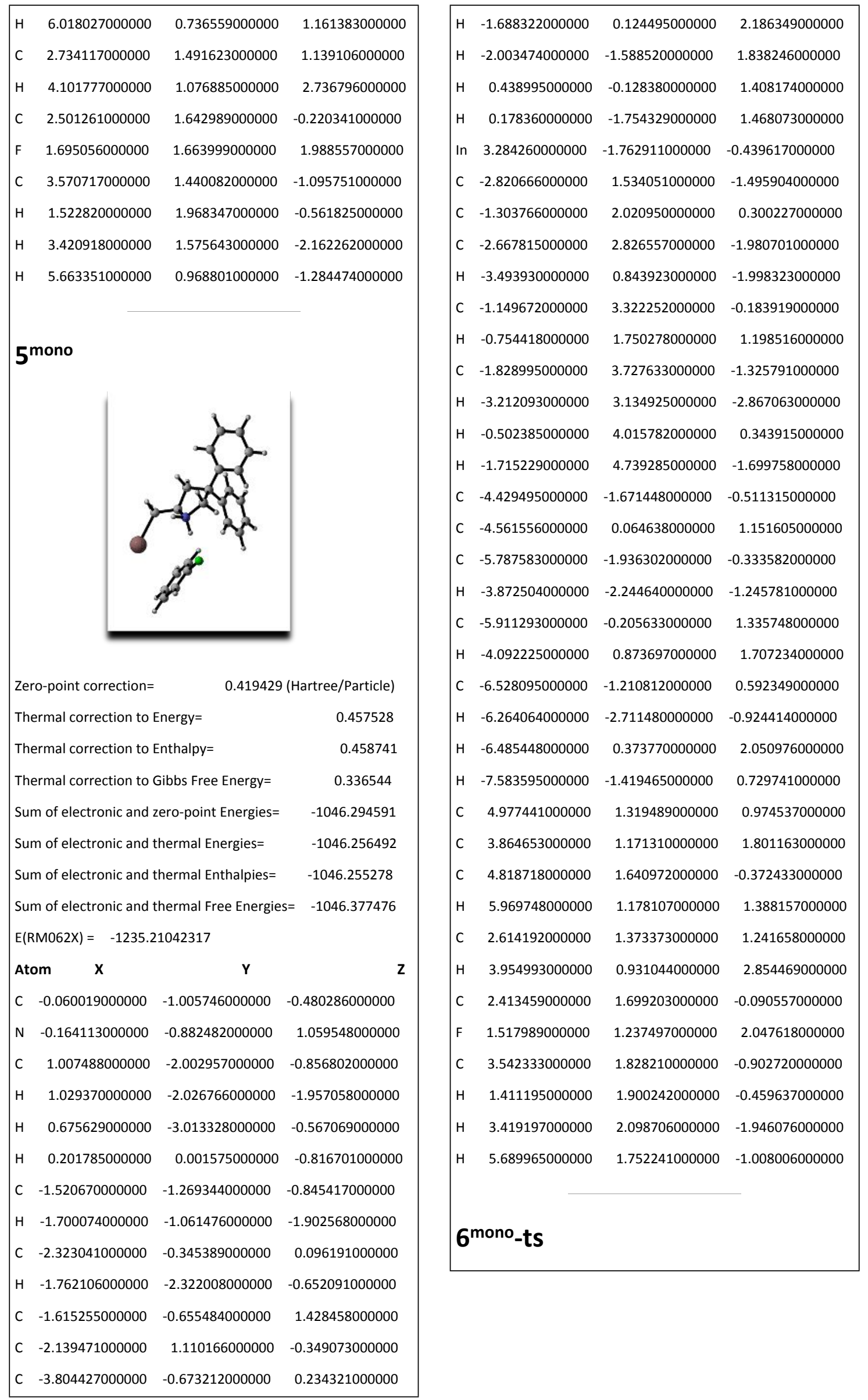




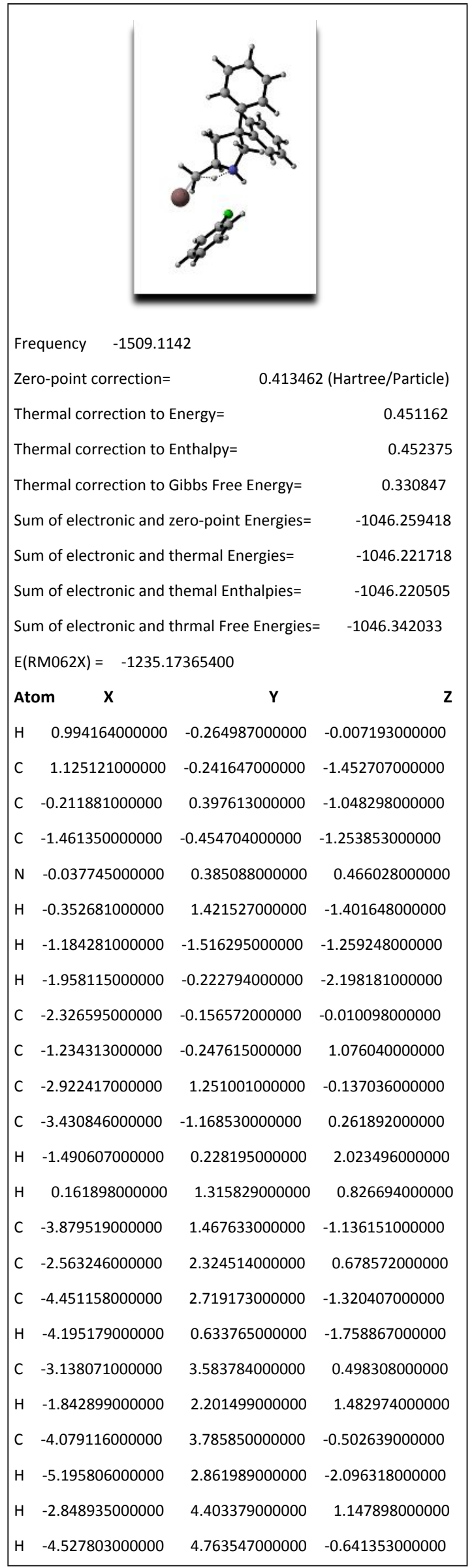

\begin{tabular}{|c|c|c|c|}
\hline c & -3.768224000000 & -2.178171000000 & -0.638839000000 \\
\hline C & -4.141220000000 & -1.071001000000 & 1.463543000000 \\
\hline c & -4.787014000000 & -3.082284000000 & -0.338624000000 \\
\hline $\mathrm{H}$ & -3.250172000000 & -2.264599000000 & -1.589055000000 \\
\hline c & -5.148884000000 & -1.976858000000 & 1.768201000000 \\
\hline $\mathrm{H}$ & -3.916195000000 & -0.262022000000 & 2.154922000000 \\
\hline C & -5.473705000000 & -2.988977000000 & 0.865997000000 \\
\hline $\mathrm{H}$ & -5.042375000000 & -3.858709000000 & -1.052356000000 \\
\hline $\mathrm{H}$ & -5.688388000000 & -1.888608000000 & 2.7054130000000 \\
\hline $\mathrm{H}$ & -6.264942000000 & -3.693635000000 & 1.098695000000 \\
\hline $\mathrm{H}$ & 0.984902000000 & -1.054872000000 & -2.176007000000 \\
\hline $\mathrm{H}$ & 1.810075000000 & 0.498652000000 & -1.886560000000 \\
\hline In & 3.175217000000 & -1.494506000000 & -0.580755000000 \\
\hline c & 4.454370000000 & 0.246532000000 & 2.047608000000 \\
\hline c & 3.260687000000 & 0.869474000000 & 1.672538000000 \\
\hline c & 5.574952000000 & 0.308206000000 & 1.218896000000 \\
\hline $\mathrm{H}$ & 4.506153000000 & -0.277289000000 & 2.996282000000 \\
\hline c & 3.228758000000 & 1.561785000000 & 0.467586000000 \\
\hline $\mathrm{H}$ & 2.384912000000 & 0.856332000000 & 2.312439000000 \\
\hline c & 4.319398000000 & 1.615887000000 & -0.391084000000 \\
\hline $\mathrm{F}$ & 2.088850000000 & 2.177511000000 & 0.103132000000 \\
\hline c & 5.503640000000 & 0.987587000000 & 0.002659000000 \\
\hline $\mathrm{H}$ & 4.244097000000 & 2.172355000000 & -1.319199000000 \\
\hline $\mathrm{H}$ & 6.372936000000 & 1.038260000000 & -0.644197000000 \\
\hline $\mathrm{H}$ & 6.500317000000 & -0.168676000000 & 1.521339000000 \\
\hline $\mathrm{H}$ & -1.018345000000 & -1.301371000000 & 1.274029000000 \\
\hline \multicolumn{4}{|c|}{$7^{\text {mono }}$} \\
\hline \multicolumn{4}{|c|}{ Zero-point correction= } \\
\hline \multicolumn{3}{|c|}{ Thermal correction to Energy= } & 0.457073 \\
\hline \multicolumn{3}{|c|}{ Thermal correction to Enthalpy= } & 0.458286 \\
\hline \multicolumn{3}{|c|}{ Thermal correction to Gibbs Free Energy= } & 0.334558 \\
\hline \multicolumn{3}{|c|}{ Sum of electronic and zero-point Energies= } & -1046.346923 \\
\hline \multicolumn{3}{|c|}{ Sum of electronic and thermal Energies= } & -1046.308712 \\
\hline \multicolumn{3}{|c|}{ Sum of electronic and thermal Enthalpies= } & -1046.307498 \\
\hline \multicolumn{3}{|c|}{ Sum of electronic and thermal Free Energies= } & $s=-1046.431227$ \\
\hline
\end{tabular}



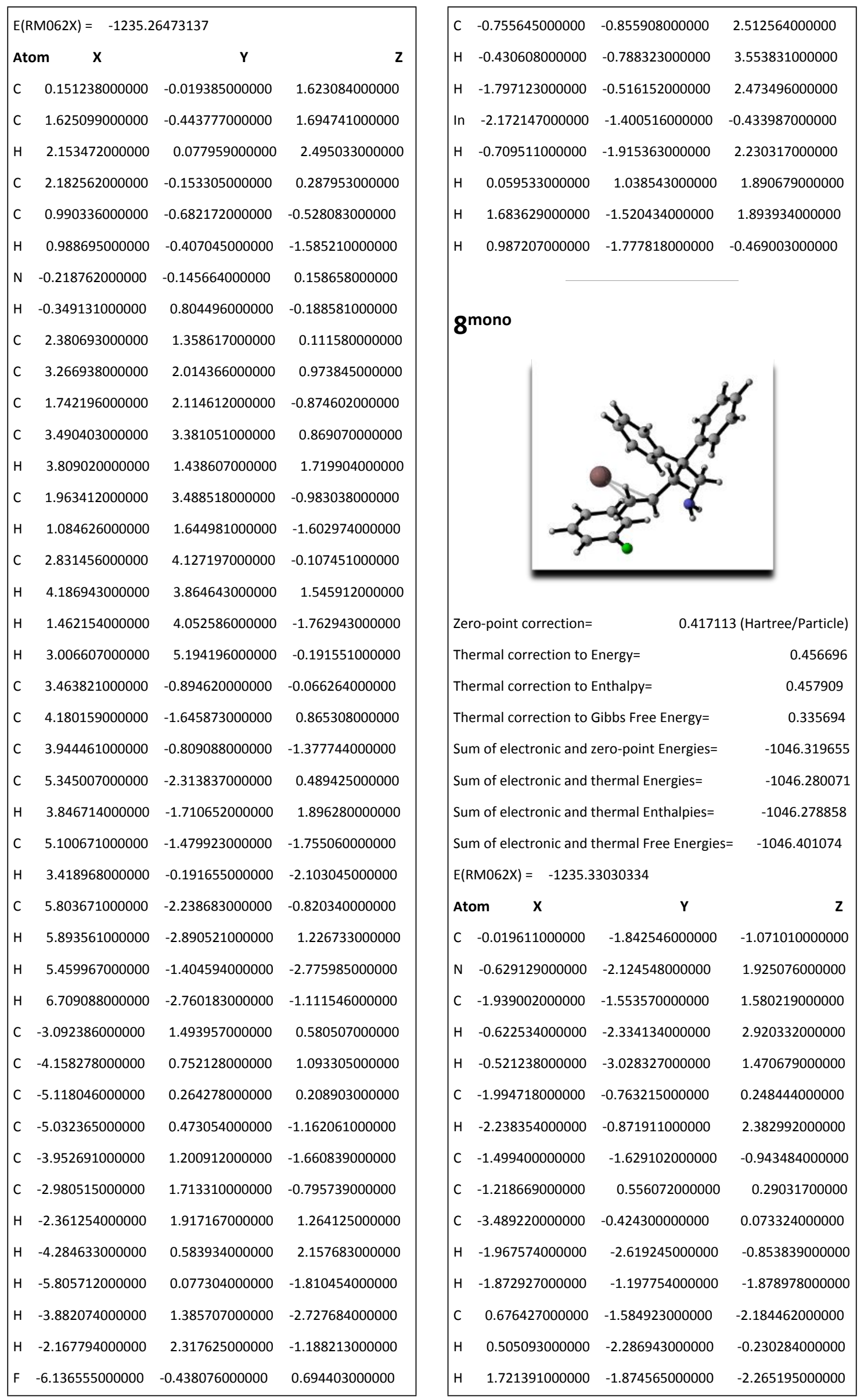


\begin{tabular}{|c|c|c|c|}
\hline $\mathrm{H}$ & 0.202564000000 & -1.169617000000 & -3.071963000000 \\
\hline C & -0.393358000000 & 0.933061000000 & 1.357622000000 \\
\hline C & -1.411755000000 & 1.485309000000 & -0.748918000000 \\
\hline C & -4.047626000000 & 0.600487000000 & 0.848101000000 \\
\hline C & -4.334361000000 & -1.156119000000 & -0.763469000000 \\
\hline C & 0.154263000000 & 2.222772000000 & 1.423017000000 \\
\hline $\mathrm{H}$ & -0.196110000000 & 0.224501000000 & 2.154461000000 \\
\hline C & -0.861324000000 & 2.764390000000 & -0.691602000000 \\
\hline $\mathrm{H}$ & -2.059633000000 & 1.221411000000 & -1.580996000000 \\
\hline C & -0.088001000000 & 3.147592000000 & 0.409767000000 \\
\hline $\mathrm{H}$ & 0.754454000000 & 2.505476000000 & 2.282564000000 \\
\hline $\mathrm{H}$ & -1.058101000000 & 3.471508000000 & -1.491079000000 \\
\hline $\mathrm{H}$ & 0.315622000000 & 4.152457000000 & 0.474443000000 \\
\hline C & -5.405048000000 & 0.890044000000 & 0.782844000000 \\
\hline $\mathrm{H}$ & -3.410760000000 & 1.188254000000 & 1.504808000000 \\
\hline C & -5.696524000000 & -0.865490000000 & -0.831234000000 \\
\hline $\mathrm{H}$ & -3.951540000000 & -1.965686000000 & -1.374728000000 \\
\hline C & -6.236484000000 & 0.157083000000 & -0.060996000000 \\
\hline $\mathrm{H}$ & -5.813808000000 & 1.690050000000 & 1.391266000000 \\
\hline $\mathrm{H}$ & -6.333171000000 & -1.446924000000 & -1.489814000000 \\
\hline $\mathrm{H}$ & -7.296207000000 & 0.381916000000 & -0.115082000000 \\
\hline In & 1.759760000000 & 0.953269000000 & -0.930563000000 \\
\hline C & 4.945055000000 & 0.067052000000 & 0.250681000000 \\
\hline $\mathrm{C}$ & 4.428923000000 & -1.114619000000 & -0.281620000000 \\
\hline C & 4.320757000000 & 0.686944000000 & 1.334962000000 \\
\hline $\mathrm{H}$ & 5.844379000000 & 0.496233000000 & 0.177831000000 \\
\hline C & 3.286512000000 & -1.656223000000 & 0.295527000000 \\
\hline $\mathrm{H}$ & 4.906689000000 & -1.628572000000 & -1.108431000000 \\
\hline C & 3.167335000000 & 0.125695000000 & 1.884777000000 \\
\hline $\mathrm{H}$ & 4.737904000000 & 1.595670000000 & 1.754366000000 \\
\hline $\mathrm{C}$ & 2.624843000000 & -1.051573000000 & 1.359276000000 \\
\hline $\mathrm{F}$ & 2.782903000000 & -2.788831000000 & -0.217273000000 \\
\hline $\mathrm{H}$ & 1.711848000000 & -1.493995000000 & 1.753897000000 \\
\hline $\mathrm{H}$ & 2.682775000000 & 0.600084000000 & 2.732533000000 \\
\hline $\mathrm{H}$ & -2.725305000000 & -2.317677000000 & 1.520908000000 \\
\hline \multicolumn{4}{|c|}{ gmono_ts } \\
\hline
\end{tabular}

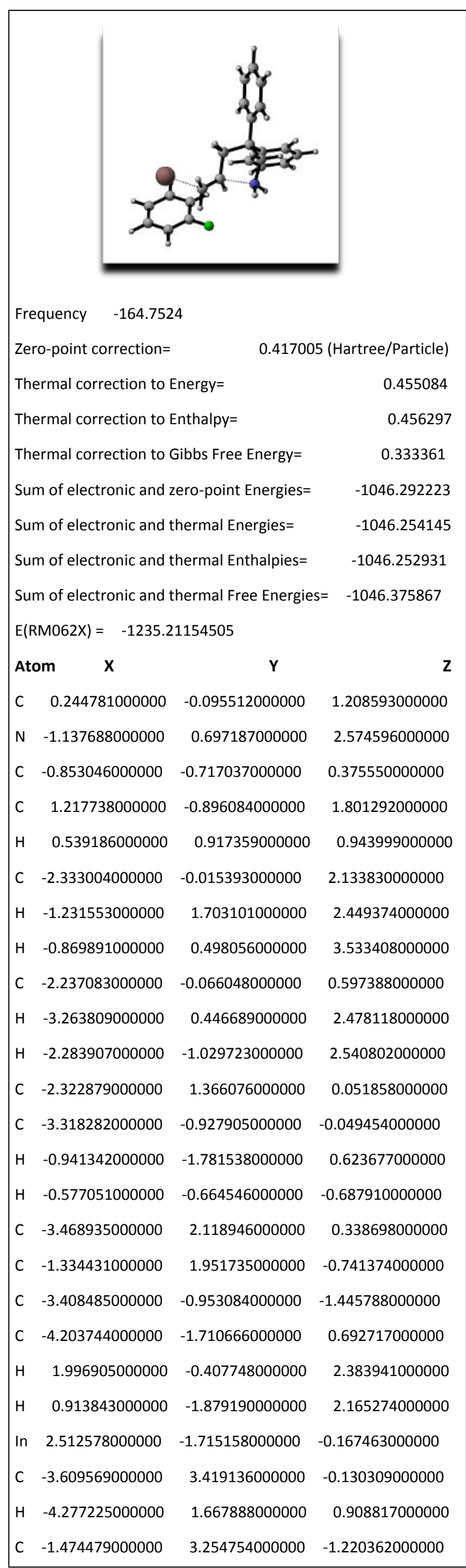




\begin{tabular}{|c|c|c|c|c|c|c|c|}
\hline $\mathrm{H}$ & -0.447080000000 & 1.390118000000 & -1.017513000000 & \multicolumn{4}{|c|}{ Sum of electronic and thermal Free Energies $=\quad-1046.381947$} \\
\hline $\mathrm{C}$ & -4.348778000000 & -1.750496000000 & -2.084560000000 & \multicolumn{4}{|c|}{$E(R M 062 X)=-1235.21702516$} \\
\hline H & -2.747368000000 & -0.322893000000 & -2.036117000000 & \multicolumn{2}{|c|}{ Atom } & $\mathbf{Y}$ & $\mathbf{Z}$ \\
\hline C & -5.153665000000 & -2.506323000000 & 0.052390000000 & C & 0.239436000000 & -0.387100000000 & 0.695205000000 \\
\hline $\mathrm{H}$ & -4.177198000000 & -1.707906000000 & 1.777732000000 & $\mathrm{~N}$ & -0.453157000000 & 0.352617000000 & 1.908144000000 \\
\hline C & -2.607726000000 & 3.995555000000 & -0.909674000000 & C & -1.884668000000 & -0.067841000000 & 1.907769000000 \\
\hline $\mathrm{H}$ & -4.508487000000 & 3.980027000000 & 0.102592000000 & $\mathrm{H}$ & -0.380159000000 & 1.365110000000 & 1.767366000000 \\
\hline H & -0.697035000000 & 3.686281000000 & -1.843080000000 & $\mathrm{H}$ & 0.045782000000 & 0.120240000000 & 2.768624000000 \\
\hline $\mathrm{H}$ & -2.718018000000 & 5.008458000000 & -1.281182000000 & C & -2.201604000000 & -0.119593000000 & 0.403714000000 \\
\hline C & -5.226457000000 & -2.532348000000 & -1.334871000000 & $\mathrm{H}$ & -2.486013000000 & 0.634044000000 & 2.487119000000 \\
\hline $\mathrm{H}$ & -4.405393000000 & -1.754997000000 & -3.168041000000 & C & -0.972741000000 & -0.921287000000 & -0.080124000000 \\
\hline $\mathrm{H}$ & -5.838298000000 & -3.103473000000 & 0.645550000000 & C & -2.235084000000 & 1.323001000000 & -0.124542000000 \\
\hline $\mathrm{H}$ & -5.967141000000 & -3.150202000000 & -1.831097000000 & C & -3.506346000000 & -0.831938000000 & 0.085049000000 \\
\hline C & 5.319491000000 & 0.267878000000 & -0.408478000000 & $\mathrm{H}$ & -1.134350000000 & -1.976161000000 & 0.163850000000 \\
\hline C & 4.575448000000 & 0.728374000000 & 0.680823000000 & $\mathrm{H}$ & -0.821684000000 & -0.856926000000 & -1.160705000000 \\
\hline C & 4.841254000000 & 0.422631000000 & -1.709630000000 & C & 1.242352000000 & -1.403635000000 & 1.127980000000 \\
\hline $\mathrm{H}$ & 6.279956000000 & -0.205599000000 & -0.234599000000 & $\mathrm{H}$ & 0.704018000000 & 0.443196000000 & 0.159537000000 \\
\hline C & 3.363407000000 & 1.361451000000 & 0.431644000000 & $\mathrm{H}$ & 1.923832000000 & -0.982845000000 & 1.879506000000 \\
\hline $\mathrm{H}$ & 4.936047000000 & 0.644593000000 & 1.700404000000 & $\mathrm{H}$ & 0.759348000000 & -2.291359000000 & 1.551869000000 \\
\hline C & 3.608530000000 & 1.037285000000 & -1.928458000000 & C & -3.230650000000 & 2.180303000000 & 0.362319000000 \\
\hline H & 5.427708000000 & 0.068964000000 & -2.549866000000 & C & -1.329376000000 & 1.824684000000 & -1.062615000000 \\
\hline C & 2.851298000000 & 1.508770000000 & -0.852573000000 & C & -3.846302000000 & -1.023131000000 & -1.258049000000 \\
\hline$F$ & 2.647956000000 & 1.821553000000 & 1.468676000000 & C & -4.374188000000 & -1.293124000000 & 1.074377000000 \\
\hline $\mathrm{H}$ & 3.234863000000 & 1.164379000000 & -2.938931000000 & C & -3.307662000000 & 3.501110000000 & -0.060039000000 \\
\hline $\mathrm{H}$ & 1.909883000000 & 2.030206000000 & -0.995423000000 & $\mathrm{H}$ & -3.974531000000 & 1.794910000000 & 1.056268000000 \\
\hline & & & & C & -1.407159000000 & 3.151225000000 & -1.491231000000 \\
\hline & O & & & $\mathrm{H}$ & -0.565359000000 & 1.185724000000 & -1.495533000000 \\
\hline & & & & C & -2.389713000000 & 3.993869000000 & -0.987198000000 \\
\hline & & & & $\mathrm{H}$ & -4.091384000000 & 4.145035000000 & 0.324585000000 \\
\hline & & & & $\mathrm{H}$ & -0.702306000000 & 3.518359000000 & -2.230392000000 \\
\hline & & & & $\mathrm{H}$ & -2.451279000000 & 5.023506000000 & -1.322360000000 \\
\hline & & & & C & -5.020063000000 & -1.679612000000 & -1.603620000000 \\
\hline & & & & $\mathrm{H}$ & -3.191068000000 & -0.638411000000 & -2.035991000000 \\
\hline & & & & C & -5.556471000000 & -1.947240000000 & 0.727892000000 \\
\hline & & & & $\mathrm{H}$ & -4.151706000000 & -1.145154000000 & 2.127491000000 \\
\hline & & & & C & -5.878901000000 & -2.145956000000 & -0.608801000000 \\
\hline & & & & $\mathrm{H}$ & -5.269454000000 & -1.823412000000 & -2.649576000000 \\
\hline & o-point correction= & 0.420053 & (Hartree/Particle) & $\mathrm{H}$ & -6.223693000000 & -2.298606000000 & 1.507764000000 \\
\hline & ermal correction to $\mathrm{E}$ & nergy= & 0.457875 & $\mathrm{H}$ & -6.797331000000 & -2.656426000000 & -0.877580000000 \\
\hline & ermal correction to $\mathrm{E}$ & nthalpy= & 0.459088 & In & 2.725069000000 & -1.931953000000 & -0.589882000000 \\
\hline & ermal correction to $\mathrm{G}$ & Sibbs Free Energy= & 0.336938 & C & 5.185722000000 & 0.596119000000 & 0.369716000000 \\
\hline & $\mathrm{n}$ of electronic and $\mathrm{z}$ & ero-point Energies= & -1046.298832 & C & 4.173431000000 & 0.775270000000 & 1.312839000000 \\
\hline & $\mathrm{m}$ of electronic and $\mathrm{t}$ & hermal Energies= & -1046.261010 & C & 5.022233000000 & 1.052051000000 & -0.938694000000 \\
\hline & $\mathrm{m}$ of electronic and $\mathrm{t}$ & hermal Enthalpies= & -1046.259797 & $\mathrm{H}$ & 6.107132000000 & 0.104895000000 & 0.663992000000 \\
\hline
\end{tabular}




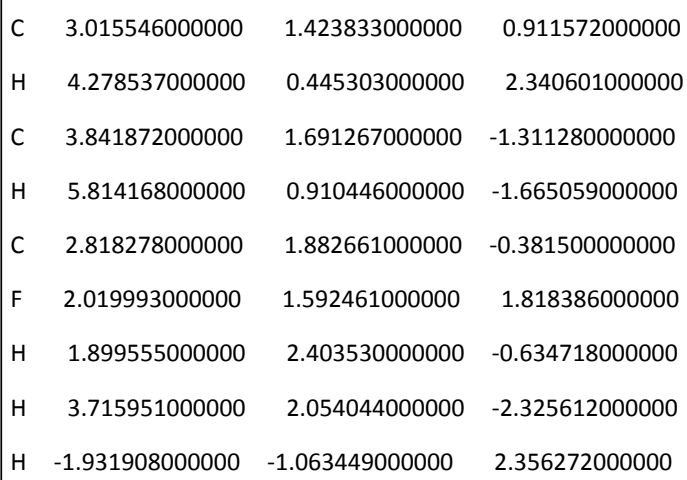

\section{$11^{\text {mono-ts }}$}

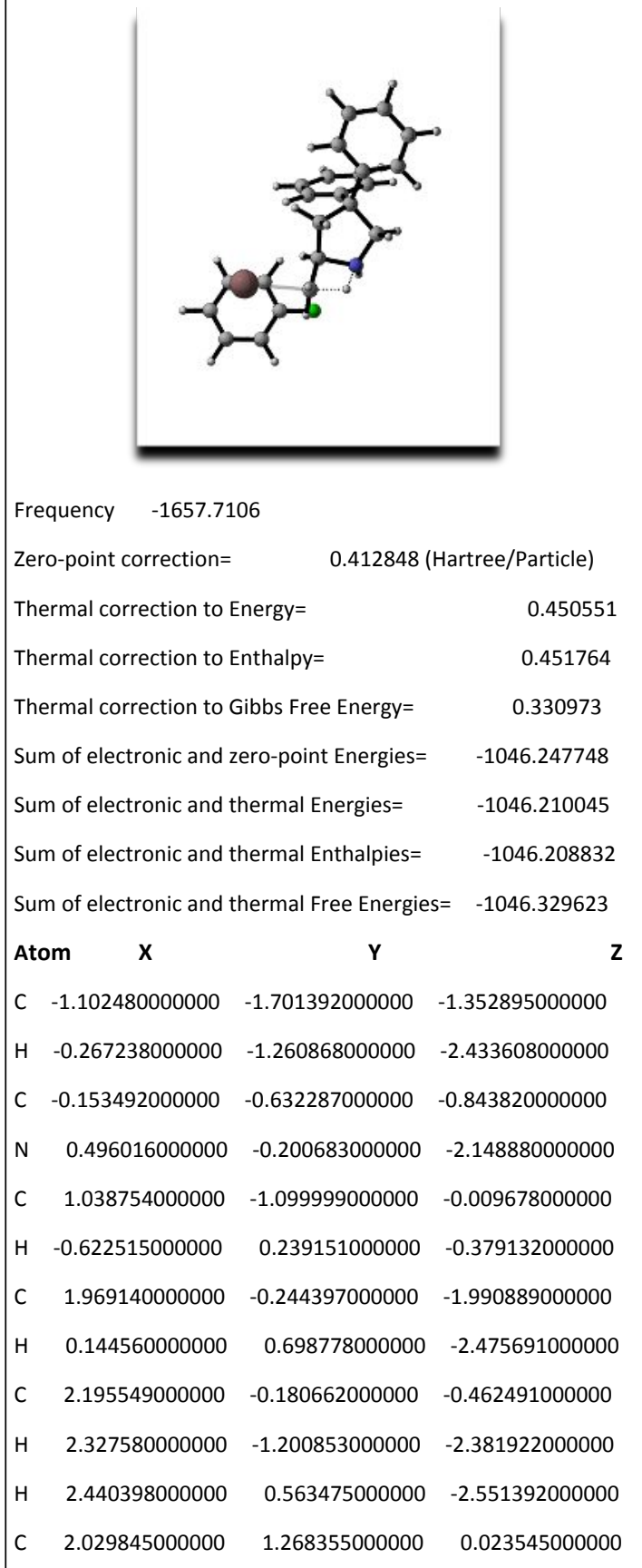

\begin{tabular}{|c|c|c|c|}
\hline C & 3.555277000000 & -0.708880000000 & -0.026265000000 \\
\hline H & 1.299884000000 & -2.133104000000 & -0.264667000000 \\
\hline H & 0.842885000000 & -1.067236000000 & 1.065396000000 \\
\hline C & 2.867650000000 & 2.250556000000 & -0.517318000000 \\
\hline C & 1.117189000000 & 1.654483000000 & 1.007590000000 \\
\hline C & 3.782192000000 & -0.952478000000 & 1.332347000000 \\
\hline C & 4.596602000000 & -0.923090000000 & -0.929859000000 \\
\hline H & -2.008996000000 & -1.411372000000 & -1.881686000000 \\
\hline H & -0.868684000000 & -2.754844000000 & -1.225578000000 \\
\hline & 2.585187000000 & -1.672623000000 & 0.712587000000 \\
\hline C & 2.775538000000 & 3.576927000000 & -0.114549000000 \\
\hline $\mathrm{H}$ & 3.625341000000 & 1.966115000000 & -1.243869000000 \\
\hline C & 1.023974000000 & 2.985006000000 & 1.419095000000 \\
\hline H & 0.470822000000 & 0.921293000000 & 1.483081000000 \\
\hline C & 5.012997000000 & -1.421392000000 & 1.773586000000 \\
\hline H & 2.993111000000 & -0.756566000000 & 2.054377000000 \\
\hline C & 5.834577000000 & -1.388478000000 & -0.487678000000 \\
\hline H & 4.461525000000 & -0.724638000000 & -1.989127000000 \\
\hline C & 1.845525000000 & 3.952311000000 & 0.853058000000 \\
\hline H & 3.439759000000 & 4.317448000000 & -0.547409000000 \\
\hline $\mathrm{H}$ & 0.315160000000 & 3.258680000000 & 2.195009000000 \\
\hline H & 1.775253000000 & 4.986246000000 & 1.172988000000 \\
\hline C & 6.044169000000 & -1.643630000000 & 0.862169000000 \\
\hline H & 5.171820000000 & -1.607763000000 & 2.830556000000 \\
\hline $\mathrm{H}$ & 6.633219000000 & -1.548624000000 & -1.204228000000 \\
\hline $\mathrm{H}$ & 7.006493000000 & -2.006732000000 & 1.206346000000 \\
\hline C & -5.162650000000 & 0.435717000000 & -0.121161000000 \\
\hline C & -4.266029000000 & 0.425011000000 & -1.192671000000 \\
\hline C & -4.833726000000 & 1.084756000000 & 1.069658000000 \\
\hline H & -6.124767000000 & -0.054444000000 & -0.227249000000 \\
\hline C & -3.054503000000 & 1.089320000000 & -1.042069000000 \\
\hline $\mathrm{H}$ & -4.505716000000 & -0.045612000000 & -2.140641000000 \\
\hline C & -3.603301000000 & 1.728810000000 & 1.194658000000 \\
\hline H & -5.537762000000 & 1.095051000000 & 1.893878000000 \\
\hline C & -2.693886000000 & 1.733851000000 & 0.134651000000 \\
\hline $\mathrm{F}$ & -2.181897000000 & 1.074509000000 & -2.068741000000 \\
\hline $\mathrm{H}$ & -3.348333000000 & 2.241446000000 & 2.116041000000 \\
\hline H & -1.738153000000 & 2.247203000000 & 0.198531000000 \\
\hline \multicolumn{4}{|c|}{$2 a$} \\
\hline
\end{tabular}




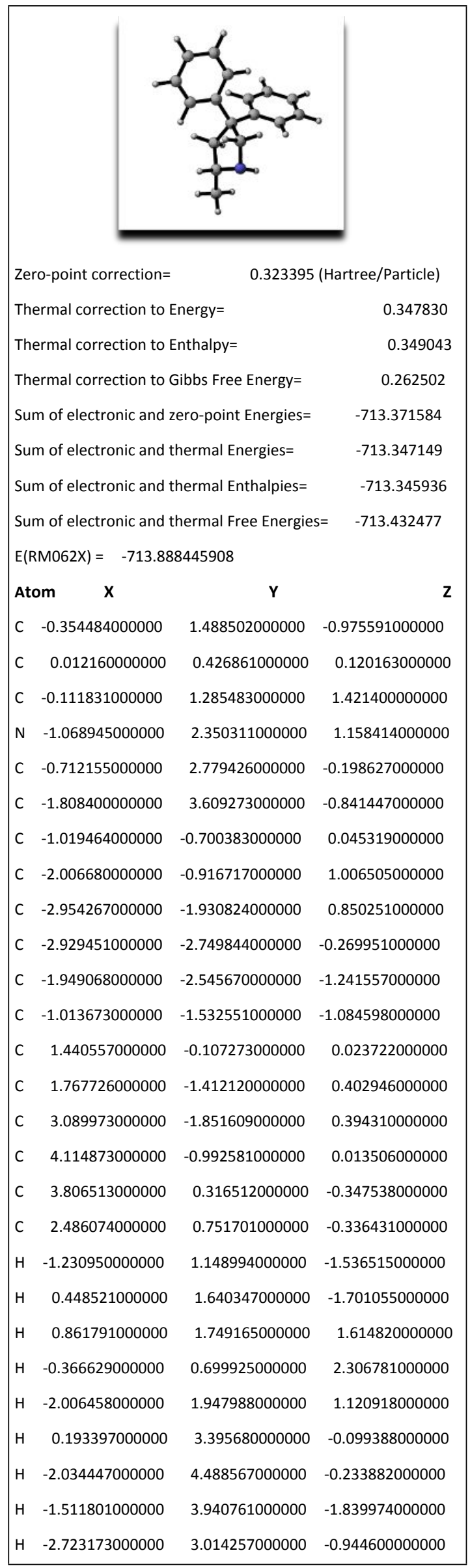

\begin{tabular}{|c|c|c|c|}
\hline $\mathrm{H}$ & -2.058307000000 & -0.299357000000 & 1.897485000000 \\
\hline $\mathrm{H}$ & -3.712002000000 & -2.075080000000 & 1.614024000000 \\
\hline $\mathrm{H}$ & -3.665142000000 & -3.538251000000 & -0.390335000000 \\
\hline $\mathrm{H}$ & -1.916803000000 & -3.177043000000 & -2.124012000000 \\
\hline $\mathrm{H}$ & -0.250796000000 & -1.379857000000 & -1.844446000000 \\
\hline $\mathrm{H}$ & 0.980273000000 & -2.095388000000 & 0.704430000000 \\
\hline $\mathrm{H}$ & 3.315923000000 & -2.872075000000 & 0.687933000000 \\
\hline $\mathrm{H}$ & 5.144155000000 & -1.336211000000 & 0.002743000000 \\
\hline $\mathrm{H}$ & 4.596326000000 & 1.002854000000 & -0.636759000000 \\
\hline $\mathrm{H}$ & 2.268321000000 & 1.783434000000 & -0.601370000000 \\
\hline \multicolumn{4}{|c|}{$2^{\text {zero }}$} \\
\hline \multicolumn{4}{|c|}{ Zero-point correction= } \\
\hline \multicolumn{3}{|c|}{ Thermal correction to Energy= } & 0.351230 \\
\hline \multicolumn{3}{|c|}{ Thermal correction to Enthalpy= } & 0.352444 \\
\hline \multicolumn{3}{|c|}{ Thermal correction to Gibbs Free Energy= } & 0.253063 \\
\hline \multicolumn{3}{|c|}{ Sum of electronic and zero-point Energies= } & -715.043795 \\
\hline \multicolumn{3}{|c|}{ Sum of electronic and thermal Energies= } & -715.015282 \\
\hline \multicolumn{3}{|c|}{ Sum of electronic and thermal Enthalpies= } & -715.014069 \\
\hline \multicolumn{3}{|c|}{ Sum of electronic and thermal Free Energies } & -715.113450 \\
\hline \multicolumn{4}{|c|}{$E(R M 062 X)=-903.765269589$} \\
\hline \multicolumn{2}{|c|}{ Atom } & $\mathbf{Y}$ & Z \\
\hline C & -3.992313000000 & -0.426611000000 & 2.347031000000 \\
\hline C & -3.021442000000 & 0.435185000000 & 2.060975000000 \\
\hline C & -1.564925000000 & 0.068560000000 & 2.055702000000 \\
\hline C & -0.897756000000 & 0.078934000000 & 0.646787000000 \\
\hline C & 0.616539000000 & -0.120054000000 & 0.896027000000 \\
\hline $\mathrm{N}$ & 1.422340000000 & -0.222274000000 & -0.352769000000 \\
\hline C & -1.020734000000 & 1.433678000000 & -0.067324000000 \\
\hline C & -1.040337000000 & 2.626182000000 & 0.666731000000 \\
\hline C & -1.035219000000 & 3.863728000000 & 0.030126000000 \\
\hline C & -0.995252000000 & 3.939858000000 & -1.359411000000 \\
\hline C & -0.962350000000 & 2.766374000000 & -2.105255000000 \\
\hline C & -0.974713000000 & 1.527471000000 & -1.465986000000 \\
\hline C & -1.489181000000 & -1.083138000000 & -0.155978000000 \\
\hline C & -0.910633000000 & -2.357810000000 & -0.155282000000 \\
\hline
\end{tabular}




\begin{tabular}{|c|c|c|c|}
\hline C & -1.509444000000 & -3.425924000000 & -0.822917000000 \\
\hline C & -2.710502000000 & -3.240412000000 & -1.496247000000 \\
\hline C & -3.311202000000 & -1.983819000000 & -1.487123000000 \\
\hline C & -2.708965000000 & -0.920315000000 & -0.824740000000 \\
\hline $\mathrm{H}$ & -5.034866000000 & -0.127496000000 & 2.347646000000 \\
\hline $\mathrm{H}$ & -3.775376000000 & -1.463044000000 & 2.593110000000 \\
\hline $\mathrm{H}$ & -3.271122000000 & 1.464543000000 & 1.809656000000 \\
\hline $\mathrm{H}$ & -1.007909000000 & 0.741160000000 & 2.721931000000 \\
\hline $\mathrm{H}$ & -1.447000000000 & -0.942107000000 & 2.462286000000 \\
\hline $\mathrm{H}$ & 0.984645000000 & 0.741641000000 & 1.463827000000 \\
\hline $\mathrm{H}$ & 0.798794000000 & -1.014984000000 & 1.500697000000 \\
\hline $\mathrm{H}$ & 1.047408000000 & -1.007216000000 & -0.896075000000 \\
\hline $\mathrm{H}$ & -1.072222000000 & 2.600104000000 & 1.751512000000 \\
\hline $\mathrm{H}$ & -1.066496000000 & 4.770988000000 & 0.624335000000 \\
\hline $\mathrm{H}$ & -0.997198000000 & 4.904473000000 & -1.855445000000 \\
\hline $\mathrm{H}$ & -0.940645000000 & 2.809173000000 & -3.189346000000 \\
\hline $\mathrm{H}$ & -0.986644000000 & 0.622336000000 & -2.067087000000 \\
\hline $\mathrm{H}$ & 0.004981000000 & -2.556380000000 & 0.397835000000 \\
\hline $\mathrm{H}$ & -1.038535000000 & -4.403299000000 & -0.803261000000 \\
\hline $\mathrm{H}$ & -3.181343000000 & -4.068778000000 & -2.014333000000 \\
\hline $\mathrm{H}$ & -4.258411000000 & -1.830183000000 & -1.993207000000 \\
\hline $\mathrm{H}$ & -3.194631000000 & 0.050058000000 & -0.818242000000 \\
\hline $\mathrm{H}$ & 1.198477000000 & 0.604637000000 & -0.918557000000 \\
\hline In & 3.723516000000 & -0.396552000000 & 0.138232000000 \\
\hline \multicolumn{4}{|c|}{$3^{\text {zero }}$} \\
\hline \multicolumn{3}{|c|}{ Zero-point correction $=$} & (Hartree/Particle) \\
\hline \multicolumn{3}{|c|}{ Thermal correction to Energy= } & 0.351310 \\
\hline \multicolumn{3}{|c|}{ Thermal correction to Enthalpy= } & 0.352523 \\
\hline \multicolumn{3}{|c|}{ Thermal correction to Gibbs Free Energy= } & 0.255004 \\
\hline \multicolumn{3}{|c|}{ Sum of electronic and zero-point Energies= } & -715.049964 \\
\hline \multicolumn{3}{|c|}{ Sum of electronic and thermal Energies= } & -715.021672 \\
\hline \multicolumn{3}{|c|}{ Sum of electronic and thermal Enthalpies= } & -715.020459 \\
\hline \multicolumn{3}{|c|}{ Sum of electronic and thermal Free Energies= } & -715.117977 \\
\hline \multicolumn{3}{|c|}{$E(R M 062 X)=-903.771534883$} & \\
\hline \multicolumn{2}{|c|}{ Atom } & $Y$ & $\mathbf{Z}$ \\
\hline
\end{tabular}

\begin{tabular}{|c|c|c|c|}
\hline C & -2.372377000000 & 0.481899000000 & 2.174005000000 \\
\hline $\mathrm{H}$ & -3.051484000000 & 1.294600000000 & 2.415311000000 \\
\hline $\mathrm{H}$ & -2.616397000000 & -0.497974000000 & 2.584277000000 \\
\hline C & -1.205526000000 & 0.706401000000 & 1.548429000000 \\
\hline $\mathrm{H}$ & -0.954543000000 & 1.724590000000 & 1.250816000000 \\
\hline C & -0.170274000000 & -0.355786000000 & 1.299194000000 \\
\hline $\mathrm{H}$ & 0.479514000000 & -0.391048000000 & 2.181491000000 \\
\hline C & 0.719063000000 & -0.168809000000 & 0.042954000000 \\
\hline C & -0.096849000000 & -0.502820000000 & -1.221007000000 \\
\hline $\mathrm{H}$ & 0.547377000000 & -0.424526000000 & -2.101261000000 \\
\hline $\mathrm{N}$ & -1.313663000000 & 0.350063000000 & -1.392572000000 \\
\hline $\mathrm{H}$ & -1.065169000000 & 1.314395000000 & -1.161611000000 \\
\hline $\mathrm{H}$ & -1.550739000000 & 0.369544000000 & -2.385396000000 \\
\hline C & 1.286165000000 & 1.258578000000 & 0.007899000000 \\
\hline C & 1.881610000000 & 1.777907000000 & 1.165095000000 \\
\hline C & 1.290285000000 & 2.055022000000 & -1.142540000000 \\
\hline C & 2.436659000000 & 3.050850000000 & 1.178882000000 \\
\hline $\mathrm{H}$ & 1.937538000000 & 1.172044000000 & 2.064796000000 \\
\hline C & 1.848901000000 & 3.334306000000 & -1.132285000000 \\
\hline $\mathrm{H}$ & 0.890219000000 & 1.682669000000 & o -2.082109000000 \\
\hline C & 2.416734000000 & 3.839811000000 & 0.029297000000 \\
\hline $\mathrm{H}$ & 2.895133000000 & 3.425490000000 & 2.087951000000 \\
\hline $\mathrm{H}$ & 1.844802000000 & 3.926779000000 & -2.041117000000 \\
\hline $\mathrm{H}$ & 2.851863000000 & 4.833144000000 & 0.039100000000 \\
\hline C & 1.882696000000 & -1.178190000000 & 0.042485000000 \\
\hline C & 1.906433000000 & -2.309236000000 & 0.861748000000 \\
\hline C & 2.934224000000 & -0.987829000000 & -0.861793000000 \\
\hline C & 2.957983000000 & -3.221628000000 & 0.782073000000 \\
\hline $\mathrm{H}$ & 1.117277000000 & -2.501179000000 & 1.580513000000 \\
\hline C & 3.978209000000 & -1.900242000000 & -0.946701000000 \\
\hline $\mathrm{H}$ & 2.948661000000 & -0.103226000000 & -1.492641000000 \\
\hline C & 3.994291000000 & -3.022768000000 & -0.121777000000 \\
\hline $\mathrm{H}$ & 2.961760000000 & -4.089619000000 & 1.432789000000 \\
\hline $\mathrm{H}$ & 4.784597000000 & -1.729387000000 & -1.651891000000 \\
\hline $\mathrm{H}$ & 4.811396000000 & -3.733486000000 & -0.181375000000 \\
\hline & -3.320316000000 & -0.468902000000 & -0.308854000000 \\
\hline $\mathrm{H}$ & -0.413522000000 & -1.550461000000 & -1.158026000000 \\
\hline $\mathrm{H}$ & -0.656526000000 & -1.341386000000 & 1.264487000000 \\
\hline \multicolumn{4}{|c|}{ 4zero_ts } \\
\hline
\end{tabular}




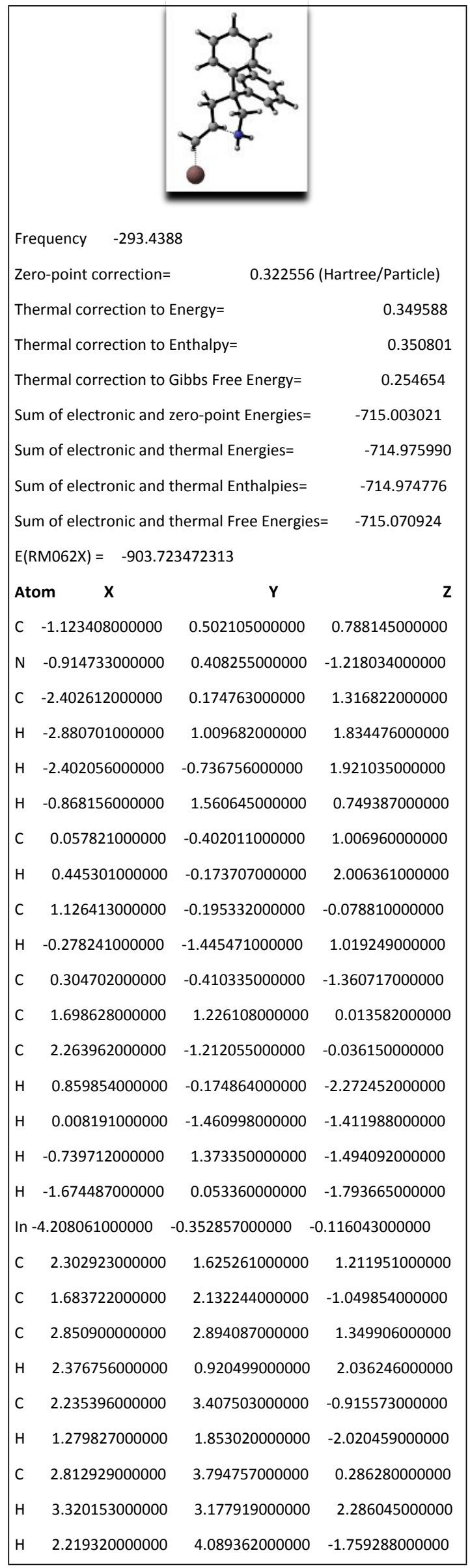

\begin{tabular}{|c|c|c|c|}
\hline $\mathrm{H}$ & 3.244730000000 & 4.783929000000 & 0.392300000000 \\
\hline C & 2.345624000000 & -2.230954000000 & 0.913306000000 \\
\hline C & 3.265493000000 & -1.115788000000 & -1.009082000000 \\
\hline C & 3.401861000000 & -3.141696000000 & 0.882132000000 \\
\hline $\mathrm{H}$ & 1.599484000000 & -2.326390000000 & 1.695746000000 \\
\hline C & 4.312250000000 & -2.027136000000 & -1.045780000000 \\
\hline $\mathrm{H}$ & 3.236933000000 & -0.302855000000 & -1.730894000000 \\
\hline C & 4.382187000000 & -3.046897000000 & -0.098254000000 \\
\hline $\mathrm{H}$ & 3.455946000000 & -3.924220000000 & 1.631779000000 \\
\hline $\mathrm{H}$ & 5.079840000000 & -1.935754000000 & -1.806858000000 \\
\hline $\mathrm{H}$ & 5.202376000000 & -3.756318000000 & -0.119769000000 \\
\hline \multicolumn{4}{|c|}{$5^{z e r o}$} \\
\hline \multicolumn{4}{|c|}{ Zero-point correction= } \\
\hline \multicolumn{3}{|c|}{ Thermal correction to Energy= } & 0.351952 \\
\hline \multicolumn{3}{|c|}{ Thermal correction to Enthalpy= } & 0.353165 \\
\hline \multicolumn{3}{|c|}{ Thermal correction to Gibbs Free Energy= } & 0.255726 \\
\hline \multicolumn{3}{|c|}{ Sum of electronic and zero-point Energies= } & -715.015256 \\
\hline \multicolumn{3}{|c|}{ Sum of electronic and thermal Energies= } & -714.987924 \\
\hline \multicolumn{3}{|c|}{ Sum of electronic and thermal Enthalpies= } & -714.986710 \\
\hline \multicolumn{3}{|c|}{ Sum of electronic and thermal Free Energies } & -715.084150 \\
\hline \multicolumn{4}{|c|}{$E(R M 062 X)=-903.735708887$} \\
\hline \multicolumn{2}{|c|}{ Atom } & $\mathbf{Y}$ & $\mathbf{Z}$ \\
\hline C & -1.045238000000 & -0.221887000000 & 0.269290000000 \\
\hline $\mathrm{N}$ & -0.747204000000 & $-0.145646000000-$ & -1.257975000000 \\
\hline C & -2.306972000000 & -1.020861000000 & 0.518918000000 \\
\hline $\mathrm{H}$ & -2.397835000000 & -1.089770000000 & 1.615069000000 \\
\hline $\mathrm{H}$ & -2.129984000000 & -2.061826000000 & 0.204180000000 \\
\hline $\mathrm{H}$ & -1.150698000000 & 0.822904000000 & 0.578763000000 \\
\hline C & 0.276938000000 & -0.770907000000 & 0.818989000000 \\
\hline $\mathrm{H}$ & 0.426365000000 & -0.434959000000 & 1.846895000000 \\
\hline C & 1.364526000000 & -0.274254000000 & -0.155437000000 \\
\hline $\mathrm{H}$ & 0.243849000000 & -1.866160000000 & 0.817770000000 \\
\hline C & 0.667285000000 & -0.628038000000 & -1.475348000000 \\
\hline C & 1.557573000000 & 1.238854000000 & 0.007143000000 \\
\hline
\end{tabular}




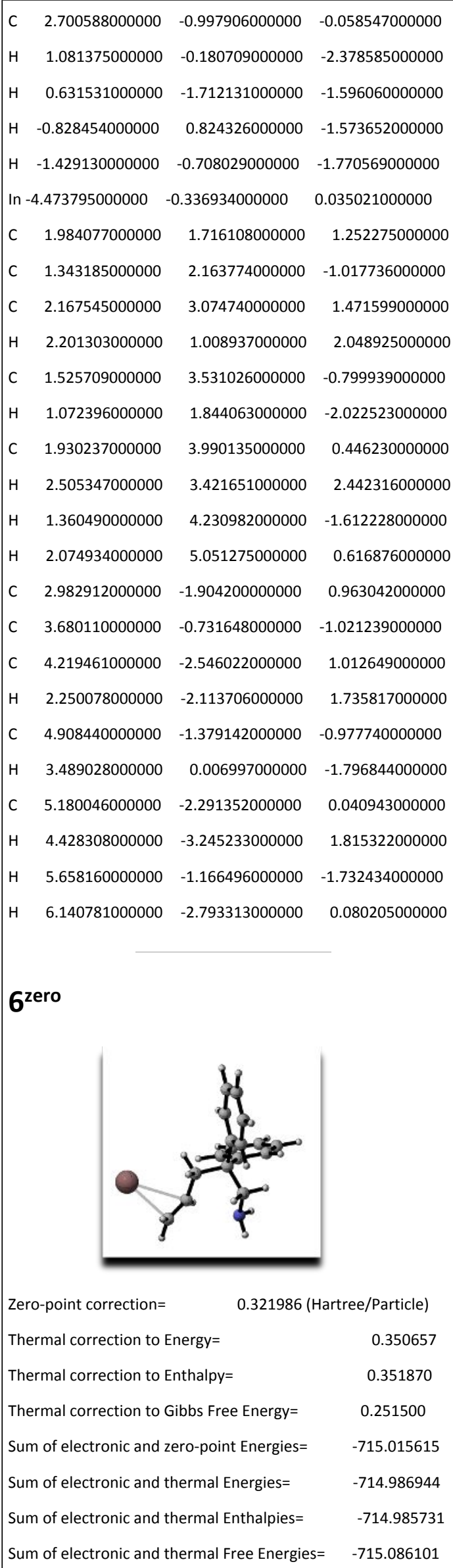

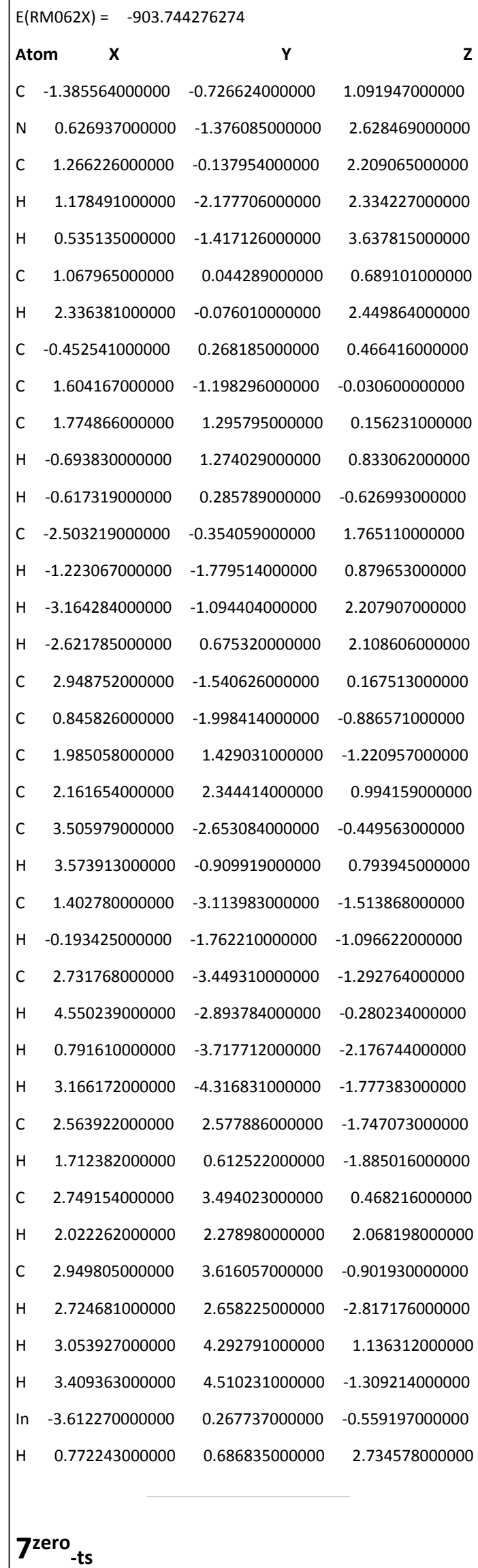




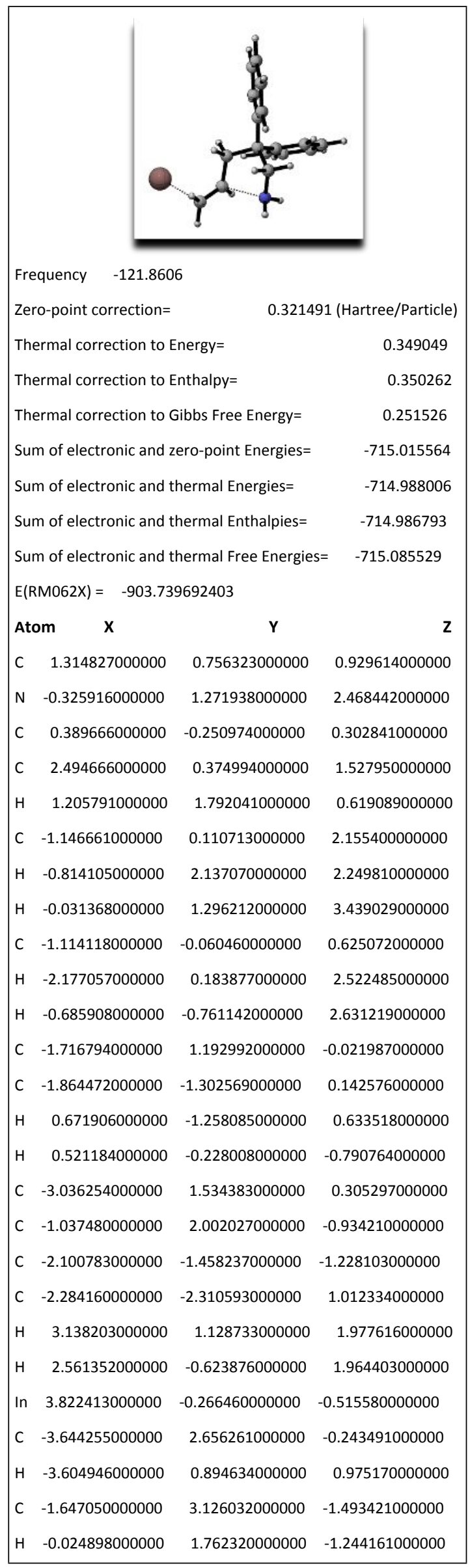

\begin{tabular}{|llll}
\hline C & -2.730453000000 & -2.595332000000 & -1.718037000000 \\
H & -1.806888000000 & -0.667409000000 & -1.914300000000 \\
C & -2.923697000000 & -3.448813000000 & 0.522303000000 \\
H & -2.131773000000 & -2.222736000000 & 2.083128000000 \\
C & -2.948562000000 & 3.461412000000 & -1.144367000000 \\
H & -4.668213000000 & 2.896687000000 & 0.022733000000 \\
H & -1.098944000000 & 3.734820000000 & -2.204973000000 \\
H & -3.423270000000 & 4.335233000000 & -1.577133000000 \\
C & -3.144868000000 & -3.596797000000 & -0.841740000000 \\
H & -2.908594000000 & -2.696048000000 & -2.783594000000 \\
H & -3.252765000000 & -4.217353000000 & 1.213892000000 \\
H & -3.643851000000 & -4.482158000000 & -1.221007000000 \\
& & & \\
8zero & &
\end{tabular}

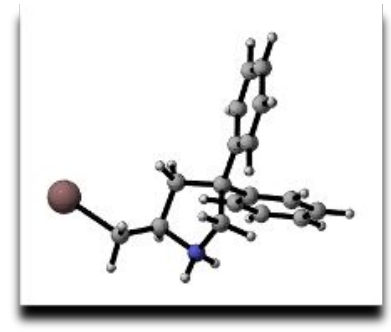

Zero-point correction=

0.324928 (Hartree/Particle)

Thermal correction to Energy=

0.352033

Thermal correction to Enthalpy=

0.353246

Thermal correction to Gibbs Free Energy=

0.256531

Sum of electronic and zero-point Energies=

$-715.025713$

Sum of electronic and thermal Energies=

$-714.998608$

Sum of electronic and thermal Enthalpies=

$-714.997395$

Sum of electronic and thermal Free Energies $=\quad-715.094110$

$E(R M 062 X)=-903.746164536$

Atom $\mathbf{X} \quad \mathrm{Y}$

C $\quad-1.157020000000-0.945384000000 \quad 0.688578000000$

N $\quad-0.150391000000 \quad-1.280180000000 \quad 1.863241000000$

C $\quad 0.816344000000 \quad-0.143192000000 \quad 1.906748000000$

H $\quad 0.368057000000 \quad-2.139646000000 \quad 1.648132000000$

$\begin{array}{llll}\text { H } & -0.667676000000 & -1.409543000000 & 2.734834000000\end{array}$

$\begin{array}{llll}\text { C } & 1.114874000000 & 0.057777000000 & 0.411616000000\end{array}$

H $\quad 1.680292000000 \quad-0.400216000000 \quad 2.521191000000$

C $\quad-0.333165000000 \quad 0.065263000000 \quad-0.135393000000$

C $\quad 1.944505000000 \quad-1.145038000000 \quad-0.060231000000$

$\begin{array}{llll}\text { C } & 1.831570000000 & 1.362412000000 & 0.101460000000\end{array}$

H $\quad-0.378715000000 \quad-0.144883000000-1.206892000000$

C $\quad-2.462082000000 \quad-0.428657000000 \quad 1.197428000000$ 


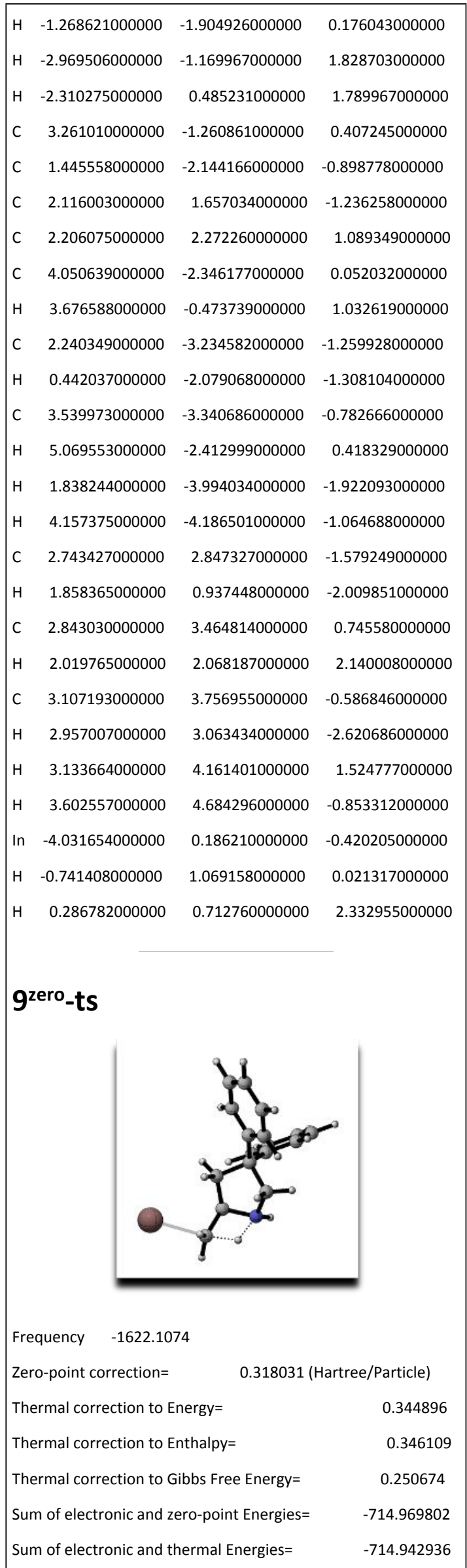

\begin{tabular}{|c|c|c|c|}
\hline \multicolumn{3}{|c|}{ Sum of electronic and thermal Enthalpies= } & -714.941723 \\
\hline \multicolumn{3}{|c|}{ Sum of electronic and thermal Free Energies= } & -715.037158 \\
\hline \multicolumn{4}{|c|}{$E(R M 062 X)=-903.686386561$} \\
\hline & $\mathrm{m}$ & $\mathbf{Y}$ & $\mathbf{Z}$ \\
\hline C & 2.442104000000 & 0.414629000000 & 1.514242000000 \\
\hline H & 1.515612000000 & 0.798071000000 & 2.485330000000 \\
\hline C & 1.188248000000 & 0.904087000000 & 0.792948000000 \\
\hline $\mathrm{N}$ & 0.293930000000 & 1.163756000000 & 2.000035000000 \\
\hline C & 0.412466000000 & -0.120130000000 & -0.057074000000 \\
\hline H & 1.350087000000 & 1.850089000000 & 0.269731000000 \\
\hline C & -0.764969000000 & 0.134708000000 & 1.959432000000 \\
\hline H & -0.103736000000 & 2.104495000000 & 2.018671000000 \\
\hline C & -1.048060000000 & -0.053087000000 & 0.453040000000 \\
\hline $\mathrm{H}$ & -0.355448000000 & -0.787679000000 & 2.387063000000 \\
\hline $\mathrm{H}$ & -1.628977000000 & 0.446645000000 & 2.547245000000 \\
\hline C & -1.806616000000 & 1.180677000000 & -0.050996000000 \\
\hline C & -1.811112000000 & -1.324244000000 & 0.111180000000 \\
\hline $\mathrm{H}$ & 0.779399000000 & -1.135138000000 & 0.141365000000 \\
\hline $\mathrm{H}$ & 0.494285000000 & 0.060239000000 & -1.132893000000 \\
\hline C & -3.114238000000 & 1.373696000000 & 0.413408000000 \\
\hline C & -1.267722000000 & 2.125357000000 & -0.925155000000 \\
\hline C & -2.022221000000 & -1.632176000000 & -1.237394000000 \\
\hline C & -2.310127000000 & -2.188053000000 & 1.085973000000 \\
\hline $\mathrm{H}$ & 3.222803000000 & 1.110857000000 & 1.813632000000 \\
\hline $\mathrm{H}$ & 2.510142000000 & -0.630619000000 & 1.818507000000 \\
\hline In & 3.815820000000 & -0.244477000000 & -0.487761000000 \\
\hline C & -3.854358000000 & 2.482303000000 & 0.026301000000 \\
\hline $\mathrm{H}$ & -3.562422000000 & 0.629747000000 & 1.068192000000 \\
\hline C & -2.011453000000 & 3.239365000000 & -1.319879000000 \\
\hline $\mathrm{H}$ & -0.267753000000 & 2.005341000000 & -1.331754000000 \\
\hline C & -2.696408000000 & -2.789907000000 & -1.603072000000 \\
\hline $\mathrm{H}$ & -1.672125000000 & -0.944004000000 & -2.003567000000 \\
\hline C & -2.993425000000 & -3.347867000000 & 0.719530000000 \\
\hline $\mathrm{H}$ & -2.186268000000 & -1.967746000000 & 2.142046000000 \\
\hline C & -3.302102000000 & 3.423766000000 & -0.842317000000 \\
\hline $\mathrm{H}$ & -4.866520000000 & 2.609091000000 & 0.395466000000 \\
\hline $\mathrm{H}$ & -1.576986000000 & 3.958351000000 & -2.006583000000 \\
\hline $\mathrm{H}$ & -3.879837000000 & 4.289038000000 & -1.148553000000 \\
\hline C & -3.182484000000 & -3.654202000000 & -0.622428000000 \\
\hline $\mathrm{H}$ & -2.853534000000 & -3.014279000000 & -2.652874000000 \\
\hline $\mathrm{H}$ & -3.380810000000 & -4.007801000000 & 1.488542000000 \\
\hline $\mathrm{H}$ & -3.715734000000 & -4.555153000000 & -0.905941000000 \\
\hline
\end{tabular}




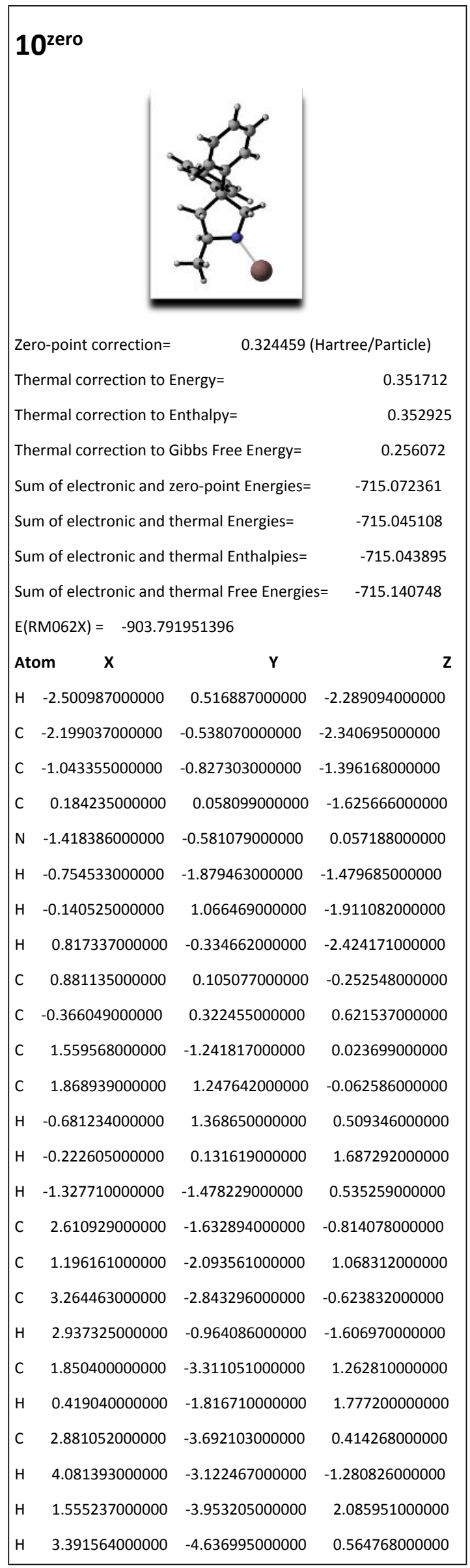

\begin{tabular}{|c|c|c|c|}
\hline C & 2.203567000000 & 2.134204000000 & -1.085658000000 \\
\hline C & 2.457928000000 & 1.413750000000 & 1.196310000000 \\
\hline C & 3.096984000000 & 3.179014000000 & -0.850410000000 \\
\hline $\mathrm{H}$ & 1.785438000000 & 2.015645000000 & -2.080238000000 \\
\hline C & 3.341622000000 & 2.458738000000 & 1.433401000000 \\
\hline $\mathrm{H}$ & 2.239681000000 & 0.701226000000 & 1.988860000000 \\
\hline C & 3.661239000000 & 3.348067000000 & 0.408446000000 \\
\hline $\mathrm{H}$ & 3.352309000000 & 3.857404000000 & -1.657621000000 \\
\hline $\mathrm{H}$ & 3.789785000000 & 2.574734000000 & 2.414593000000 \\
\hline $\mathrm{H}$ & 4.356056000000 & 4.160969000000 & 0.589807000000 \\
\hline $\mathrm{H}$ & -1.896399000000 & -0.724530000000 & -3.373986000000 \\
\hline $\mathrm{H}$ & -3.066061000000 & -1.179043000000 & -2.142516000000 \\
\hline In & -3.528675000000 & 0.392686000000 & 0.478162000000 \\
\hline
\end{tabular}

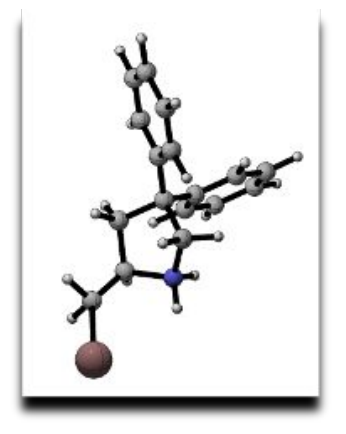

Frequency $\quad-48.1832$

Zero-point correction=

0.324809 (Hartree/Particle)

Thermal correction to Energy=

0.350911

Thermal correction to Enthalpy=

0.352124

Thermal correction to Gibbs Free Energy=

0.258678

Sum of electronic and zero-point Energies=

$-715.012130$

Sum of electronic and thermal Energies=

Sum of electronic and thermal Enthalpies=

$-714.986027$

Sum of electronic and thermal Free Energies=

$-714.984814$

$E(R M 062 X)=-903.733610184$

Atom $\mathbf{X}$

$\mathbf{Y}$

Z

C $\quad 1.021712000000 \quad-0.845203000000 \quad 0.898030000000$

N $\quad 0.718418000000 \quad-1.130005000000 \quad-0.612407000000$

C $\quad-0.145189000000-0.004207000000-1.076746000000$

H $\quad 0.171143000000 \quad-1.996341000000 \quad-0.691240000000$

H $\quad 1.584467000000 \quad-1.235889000000-1.139458000000$

C $\quad-1.159311000000 \quad 0.078939000000 \quad 0.077482000000$

H $\quad-0.572363000000 \quad-0.228625000000 \quad-2.055544000000$

C $\quad-0.178884000000 \quad 0.056223000000 \quad 1.276212000000$

C $\quad-2.056527000000-1.163852000000 \quad-0.013431000000$ 


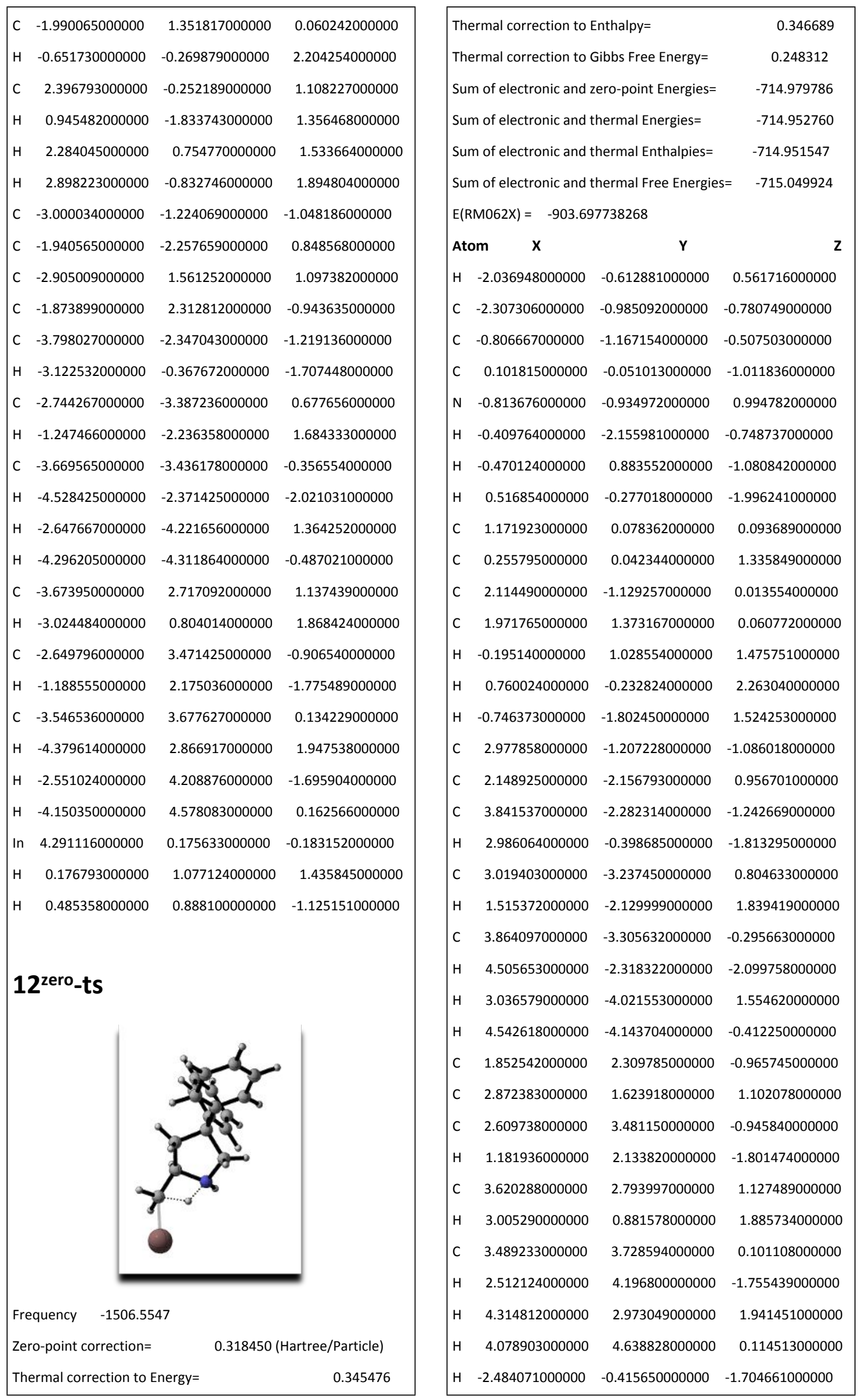




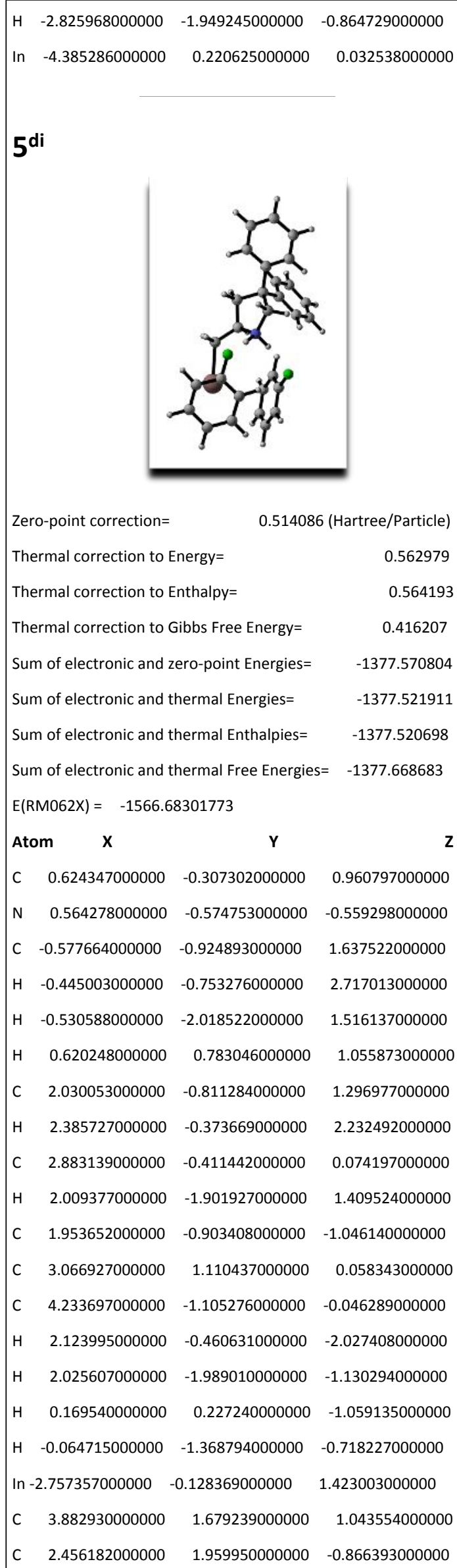

\begin{tabular}{|c|c|c|c|}
\hline C & 4.074571000000 & 3.052755000000 & 1.108876000000 \\
\hline $\mathrm{H}$ & 4.390789000000 & 1.028576000000 & 1.751467000000 \\
\hline C & 2.648808000000 & 3.342580000000 & -0.804666000000 \\
\hline $\mathrm{H}$ & 1.826803000000 & 1.575002000000 & -1.665977000000 \\
\hline C & 3.454103000000 & 3.892544000000 & 0.184203000000 \\
\hline $\mathrm{H}$ & 4.718109000000 & 3.469832000000 & 1.876298000000 \\
\hline $\mathrm{H}$ & 2.171631000000 & 3.982569000000 & -1.540284000000 \\
\hline $\mathrm{H}$ & 3.609035000000 & 4.965051000000 & 0.229176000000 \\
\hline C & 4.726450000000 & -1.974443000000 & 0.926187000000 \\
\hline C & 5.005896000000 & -0.853497000000 & -1.185720000000 \\
\hline C & 5.965807000000 & -2.591946000000 & 0.754939000000 \\
\hline $\mathrm{H}$ & 4.158908000000 & -2.172939000000 & 1.829958000000 \\
\hline C & 6.235367000000 & -1.475238000000 & -1.360811000000 \\
\hline $\mathrm{H}$ & 4.648340000000 & -0.145468000000 & -1.930196000000 \\
\hline C & 6.718171000000 & -2.349638000000 & -0.388167000000 \\
\hline $\mathrm{H}$ & 6.340836000000 & -3.262137000000 & 1.521255000000 \\
\hline $\mathrm{H}$ & 6.821862000000 & -1.270152000000 & -2.250124000000 \\
\hline $\mathrm{H}$ & 7.681285000000 & -2.831164000000 & -0.519014000000 \\
\hline C & -3.881758000000 & 2.826372000000 & -0.834219000000 \\
\hline C & -3.039039000000 & 2.105073000000 & -1.677985000000 \\
\hline C & -3.370980000000 & 3.466873000000 & 0.293516000000 \\
\hline $\mathrm{H}$ & -4.940354000000 & 2.886647000000 & -1.061717000000 \\
\hline C & -1.691962000000 & 2.059098000000 & -1.362264000000 \\
\hline $\mathrm{H}$ & -3.399639000000 & 1.615299000000 & -2.575830000000 \\
\hline C & -1.145301000000 & 2.686658000000 & -0.254289000000 \\
\hline $\mathrm{F}$ & -0.858782000000 & 1.351425000000 & -2.182228000000 \\
\hline C & -2.008808000000 & 3.398324000000 & 0.580726000000 \\
\hline $\mathrm{H}$ & -0.071835000000 & 2.665422000000 & -0.085570000000 \\
\hline $\mathrm{H}$ & -1.606737000000 & 3.913076000000 & 1.446900000000 \\
\hline $\mathrm{H}$ & -4.033030000000 & 4.026951000000 & 0.943997000000 \\
\hline C & -5.140623000000 & -2.101410000000 & -0.762289000000 \\
\hline C & -4.337925000000 & -1.349378000000 & -1.616071000000 \\
\hline C & -2.963810000000 & -1.587825000000 & -1.690501000000 \\
\hline C & -2.441813000000 & -2.603854000000 & -0.905478000000 \\
\hline C & -3.204739000000 & -3.355134000000 & -0.026539000000 \\
\hline C & -4.573360000000 & -3.094321000000 & 0.035720000000 \\
\hline $\mathrm{H}$ & -6.206326000000 & -1.910368000000 & -0.712948000000 \\
\hline $\mathrm{H}$ & -4.780242000000 & -0.574638000000 & -2.233056000000 \\
\hline $\mathrm{H}$ & -2.318879000000 & -1.023221000000 & -2.357696000000 \\
\hline $\mathrm{H}$ & -2.735473000000 & -4.133801000000 & 0.564255000000 \\
\hline $\mathrm{H}$ & -5.196028000000 & -3.676825000000 & 0.705908000000 \\
\hline $\mathrm{F}$ & -1.108617000000 & -2.866590000000 & -0.985687000000 \\
\hline
\end{tabular}




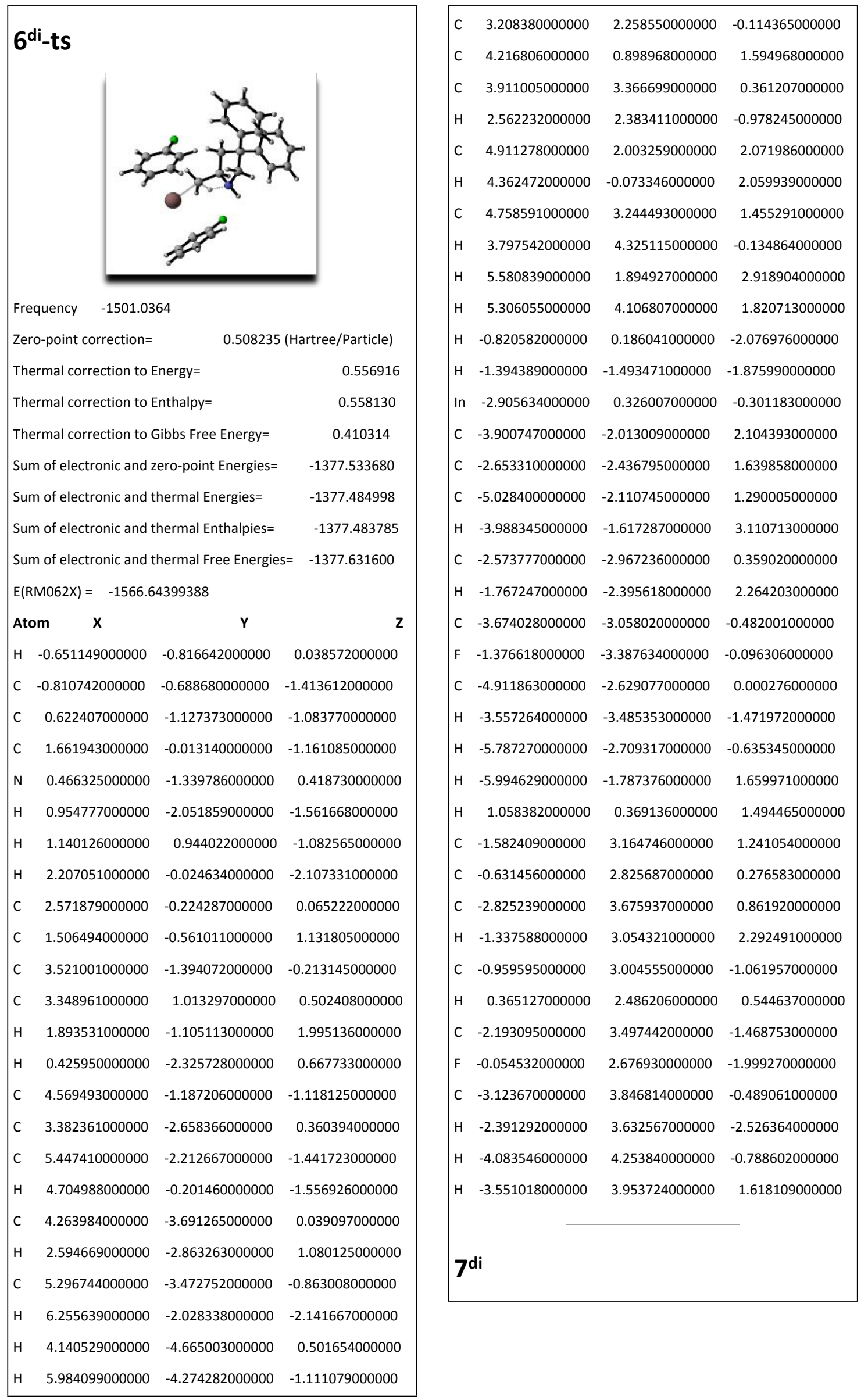




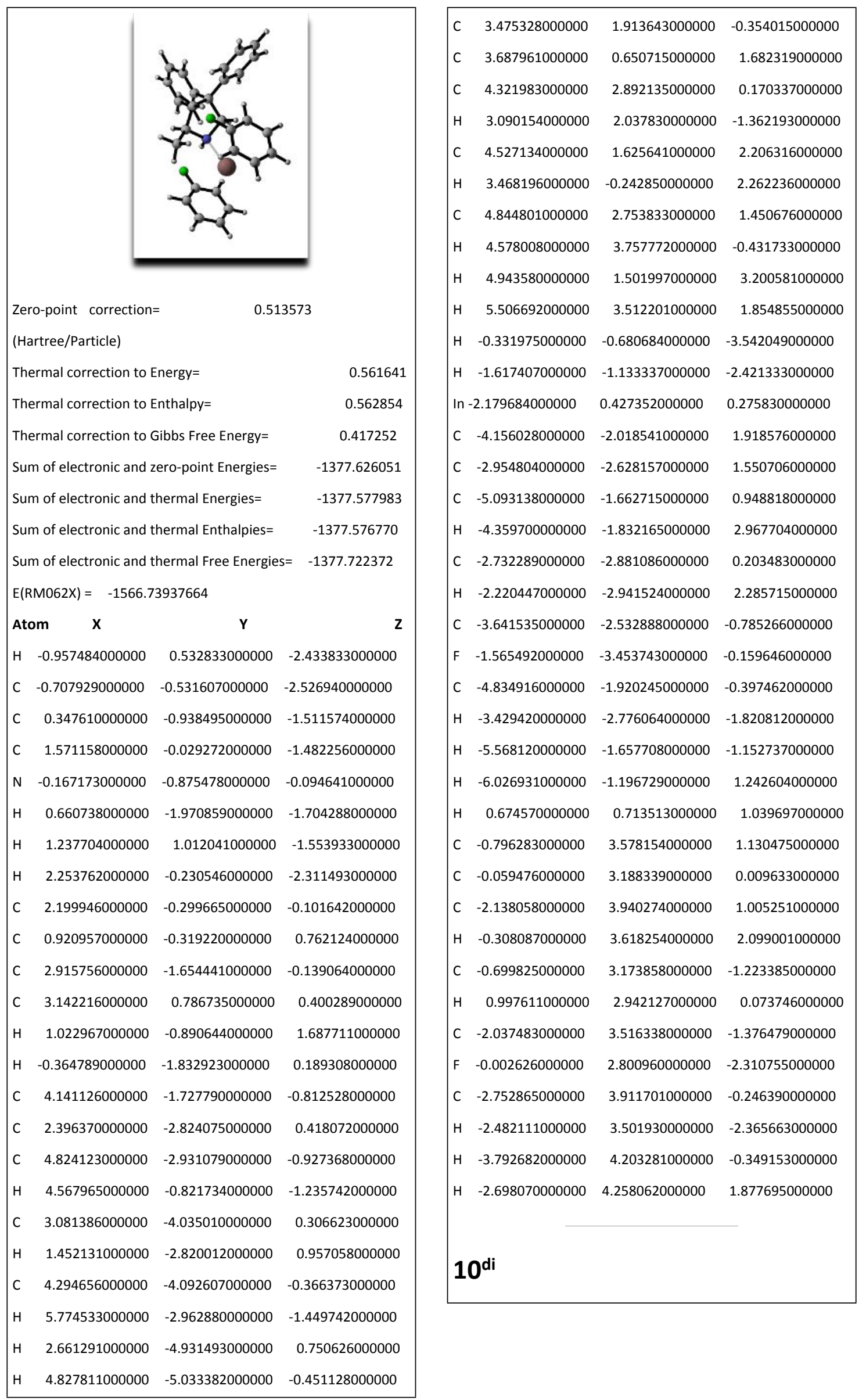




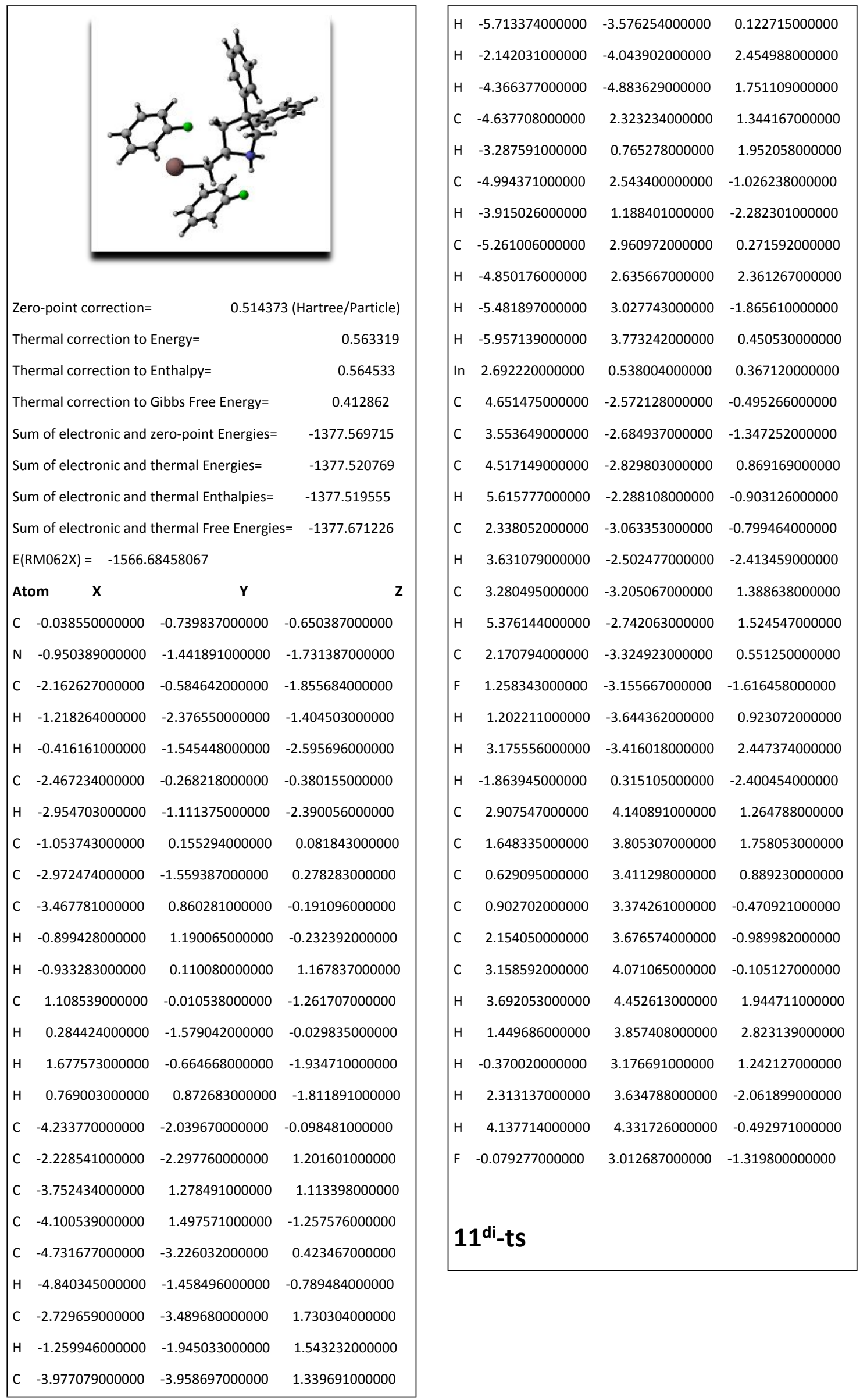




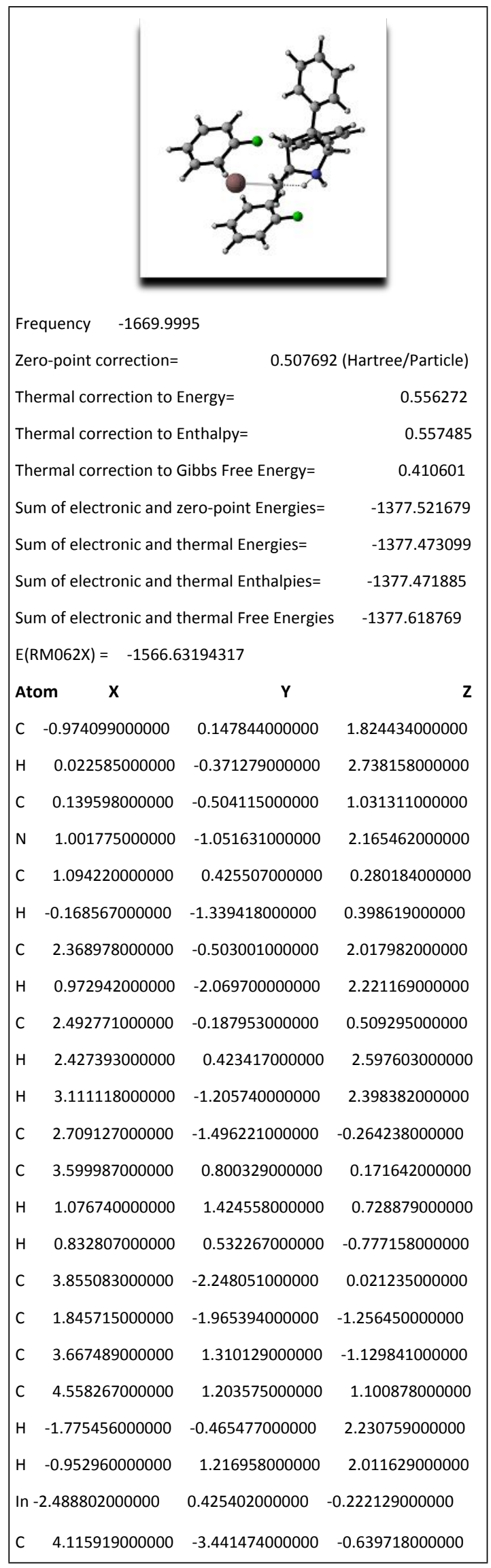

\begin{tabular}{|c|c|c|c|}
\hline $\mathrm{H}$ & 4.567911000000 & -1.876310000000 & 0.753634000000 \\
\hline$C$ & 2.106289000000 & -3.161837000000 & -1.926822000000 \\
\hline $\mathrm{H}$ & 0.960664000000 & -1.400196000000 & -1.536664000000 \\
\hline$C$ & 4.654895000000 & 2.219000000000 & -1.486765000000 \\
\hline $\mathrm{H}$ & 2.952220000000 & 0.969099000000 & -1.875176000000 \\
\hline$C$ & 5.553892000000 & 2.112462000000 & 0.742643000000 \\
\hline $\mathrm{H}$ & 4.547110000000 & 0.811565000000 & 2.113494000000 \\
\hline$C$ & 3.236373000000 & -3.907850000000 & -1.615384000000 \\
\hline $\mathrm{H}$ & 5.012132000000 & -4.004124000000 & -0.400782000000 \\
\hline $\mathrm{H}$ & 1.426331000000 & -3.502024000000 & -2.702030000000 \\
\hline $\mathrm{H}$ & 3.439458000000 & -4.837511000000 & -2.135731000000 \\
\hline$C$ & 5.601391000000 & 2.626416000000 & -0.547334000000 \\
\hline $\mathrm{H}$ & 4.694707000000 & 2.603515000000 & -2.500670000000 \\
\hline $\mathrm{H}$ & 6.292655000000 & 2.415596000000 & 1.477249000000 \\
\hline $\mathrm{H}$ & 6.376252000000 & 3.332938000000 & -0.825024000000 \\
\hline$C$ & -4.492555000000 & -2.501526000000 & 0.192392000000 \\
\hline C & -3.525997000000 & -2.552307000000 & 1.198221000000 \\
\hline C & -4.147945000000 & -2.768506000000 & -1.133138000000 \\
\hline $\mathrm{H}$ & -5.519622000000 & -2.266145000000 & 0.451215000000 \\
\hline C & -2.225480000000 & -2.883720000000 & 0.842358000000 \\
\hline $\mathrm{H}$ & -3.768363000000 & -2.376060000000 & 2.241009000000 \\
\hline C & -2.830963000000 & -3.088513000000 & -1.459458000000 \\
\hline $\mathrm{H}$ & -4.906254000000 & -2.735028000000 & -1.907340000000 \\
\hline C & -1.849635000000 & -3.145371000000 & -0.468200000000 \\
\hline $\mathrm{F}$ & -1.284285000000 & -2.921809000000 & 1.807243000000 \\
\hline $\mathrm{H}$ & -2.563176000000 & -3.306367000000 & -2.487881000000 \\
\hline $\mathrm{H}$ & -0.819327000000 & -3.410266000000 & -0.689218000000 \\
\hline C & -3.461266000000 & 3.735786000000 & -0.227670000000 \\
\hline C & -2.500948000000 & 3.602819000000 & 0.777357000000 \\
\hline C & -3.090674000000 & 3.676981000000 & -1.571885000000 \\
\hline $\mathrm{H}$ & -4.498566000000 & 3.900804000000 & 0.044571000000 \\
\hline C & -1.174574000000 & 3.425682000000 & 0.401972000000 \\
\hline $\mathrm{H}$ & -2.753641000000 & 3.672525000000 & 1.830051000000 \\
\hline C & -1.754422000000 & 3.478531000000 & -1.916853000000 \\
\hline $\mathrm{H}$ & -3.840082000000 & 3.793529000000 & -2.346438000000 \\
\hline C & -0.778884000000 & 3.341348000000 & -0.926716000000 \\
\hline$F$ & -0.246073000000 & 3.302870000000 & 1.365034000000 \\
\hline $\mathrm{H}$ & -1.462503000000 & 3.439792000000 & -2.960938000000 \\
\hline $\mathrm{H}$ & 0.272249000000 & 3.212844000000 & -1.164340000000 \\
\hline
\end{tabular}




\section{References}

(1) Zhao, Y.; Truhlar, D. G. The M06 Suite of Density Functionals for Main Group Thermochemistry, Thermochemical Kinetics, Noncovalent Interactions, Excited States, and Transition Elements: Two New Functionals and Systematic Testing of Four M06-Class Functionals and 12 Other Function. Theor. Chem. Acc. 2008, 120, 215-241.

(2) Frisch, M. J.; T., G. W.; Schlegel, H. B.; Scuseria, G. E.; Robb, M. A.; Cheeseman, J. R.; Scalmani, G.; Barone, V.; Mennucci, B.; Petersson, G. A.; Nakatsuji, H.; Caricato, M.; Li, X.; Hratchian, H. P.; Izmaylov, A. F.; Bloino, J.; Zheng, G.; Sonnenberg; Hada, J. L.; J., F. D. Gaussian 09, Revision A. 02. Gaussian, Inc., Wallingford, CT, 2009

(3) Krishnan, R.; Binkley, J. S.; Seeger, R.; Pople, J. A. Self-Consistent Molecular Orbital Methods. XX. A Basis Set for Correlated Wave Functions. J. Chem. Phys. 1980, 72, 650-654.

(4) Hay, P. J.; Wadt, W. R. Ab Initio Effective Core Potentials for Molecular Calculations. Potentials for K to Au Including the Outermost Core Orbitale. J. Chem. Phys. 1985, 82, 299-310.

(5) Hehre, W. J.; Ditchfield, K.; Pople, J. A. Self-Consistent Molecular Orbital Methods. XII. Further Extensions of Gaussian-Type Basis Sets for Use in Molecular Orbital Studies of Organic Molecules. J. Chem. Phys. 1972, 56, 2257-2261.

(6) Weigend, F.; Ahlrichs, R. Balanced Basis Sets of Split Valence, Triple Zeta Valence and Quadruple Zeta Valence Quality for H to Rn: Design and Assessment of Accuracy. Phys. Chem. Chem. Phys. 2005, 7, 3297-3305.

(7) Marenich, A. V.; Cramer, C. J.; Truhlar, D. G. Universal Solvation Model Based on Solute Electron Density and on a Continuum Model of the Solvent Defined by the Bulk Dielectric Constant and Atomic Surface Tensions. J. Phys. Chem. B 2009, 113, 6378-6396.

(8) Fukui, K. The Path of Chemical Reactions - The IRC Approach. Acc. Chem. Res. 1981, 14, 363-368.

(9) Fukui, K. A Formulation of the Reaction Coordinate. J. Phys. Chem. 1970, 74, 4161.

(10) CYLview Visualization Software http://www.cylview.org./ (accessed Apr 15, 2020). 


\section{4. ${ }^{1} \mathrm{H},{ }^{13} \mathrm{C},{ }^{19} \mathrm{~F}$ NMR spectral data}

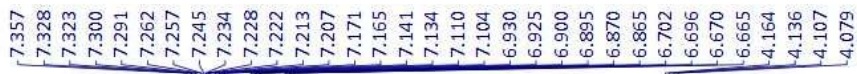
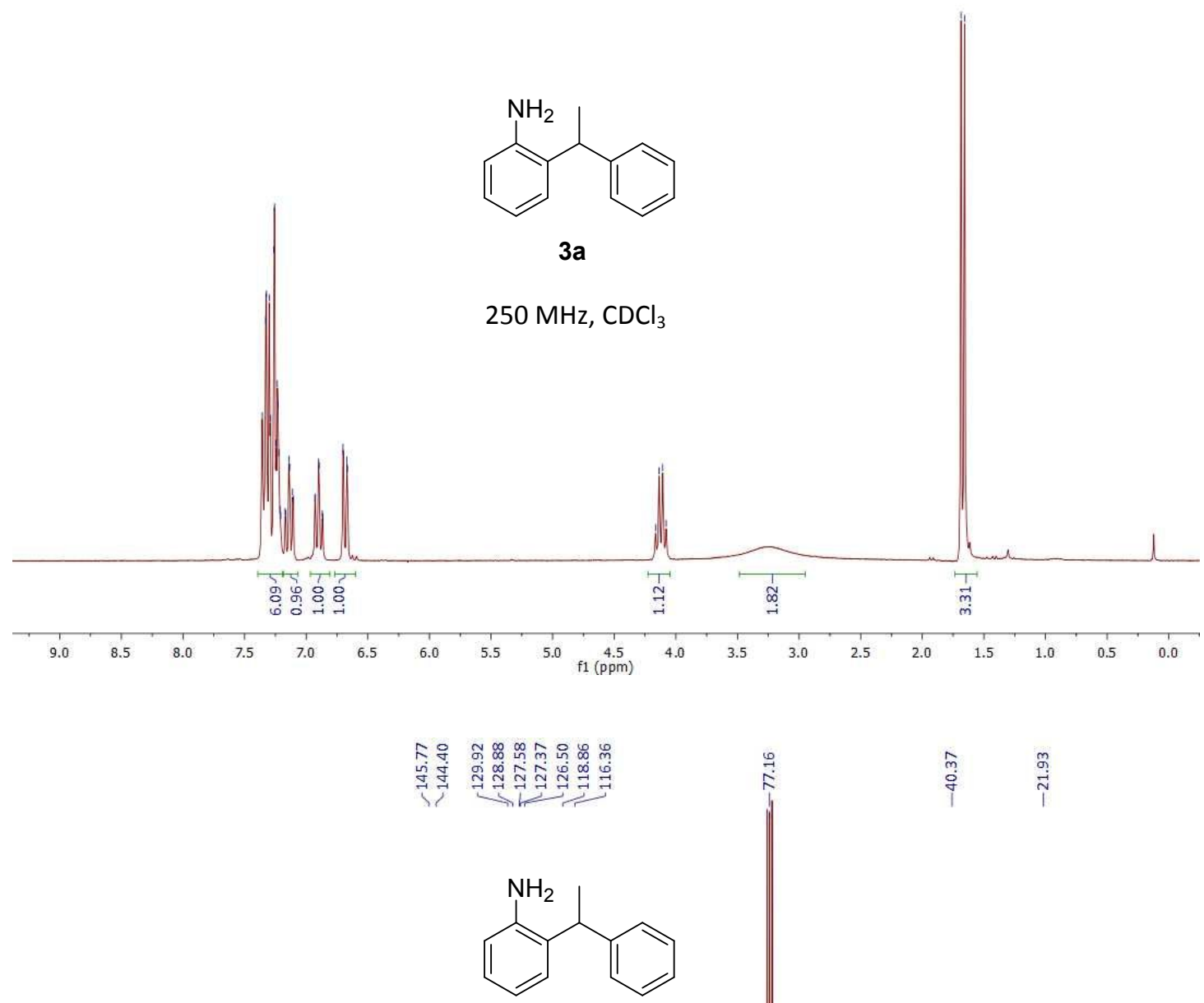

$3 a$

$63 \mathrm{MHz}, \mathrm{CDCl}_{3}$

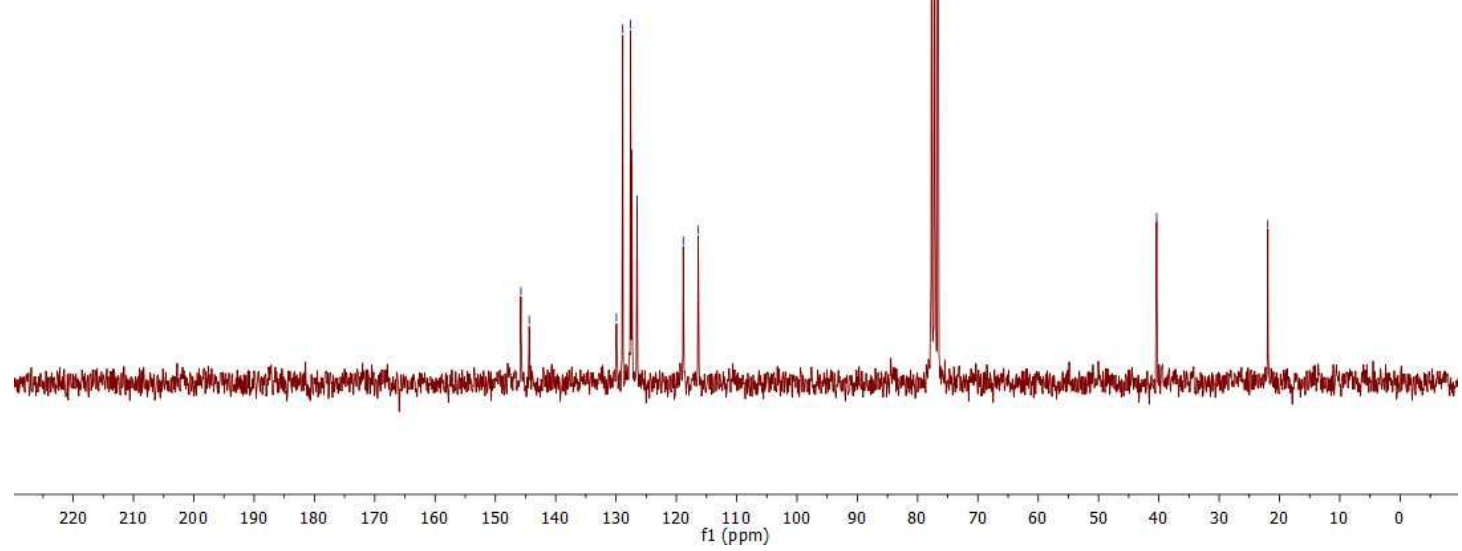




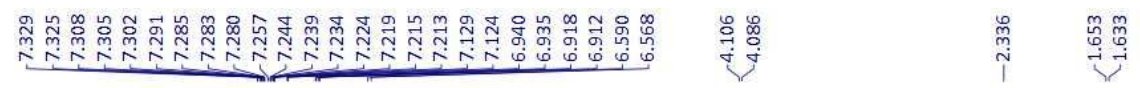

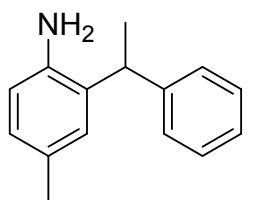

$3 b$

$360 \mathrm{MHz}, \mathrm{CDCl}_{3}$

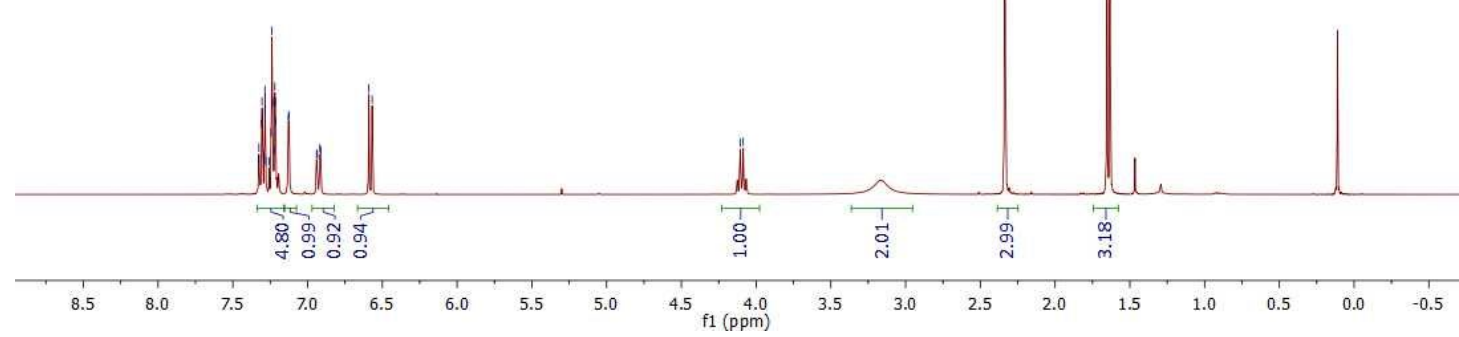

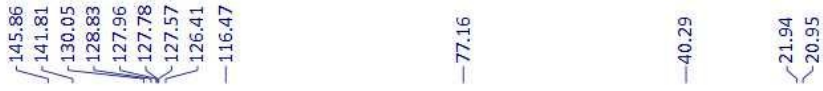

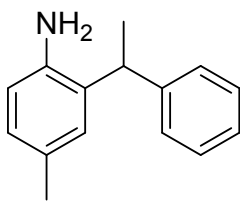

$3 b$

$91 \mathrm{MHz}, \mathrm{CDCl}_{3}$

$\begin{array}{lllllllllllllllllllllll}210 & 200 & 190 & 180 & 170 & 160 & 150 & 140 & 130 & 120 & 110 & \begin{array}{c}100 \\ \mathrm{f} 1(\mathrm{ppm})\end{array} & 90 & 80 & 70 & 60 & 50 & 40 & 30 & 20 & 10 & 0 & -10\end{array}$ 

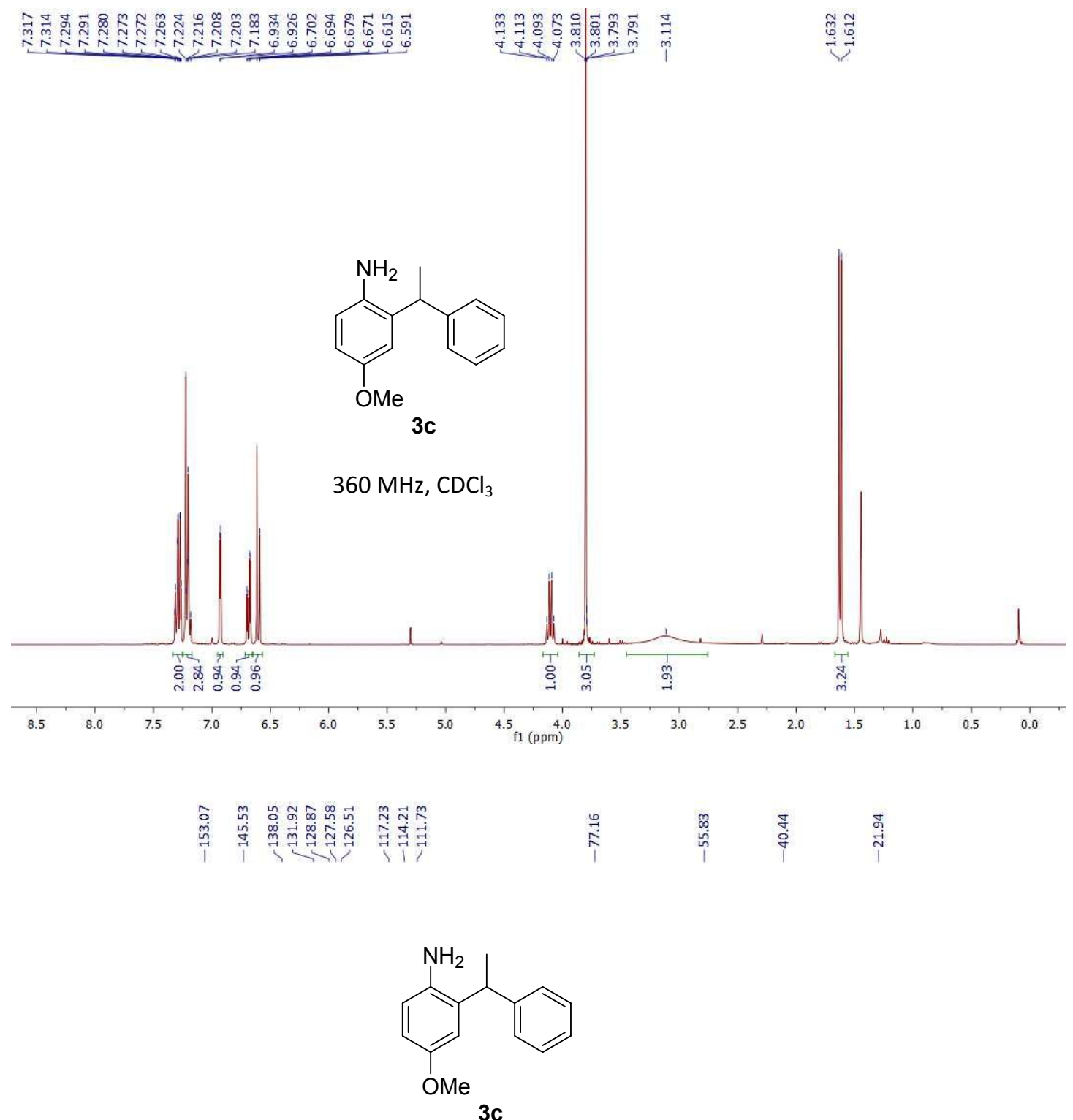

$91 \mathrm{MHz}, \mathrm{CDCl}_{3}$

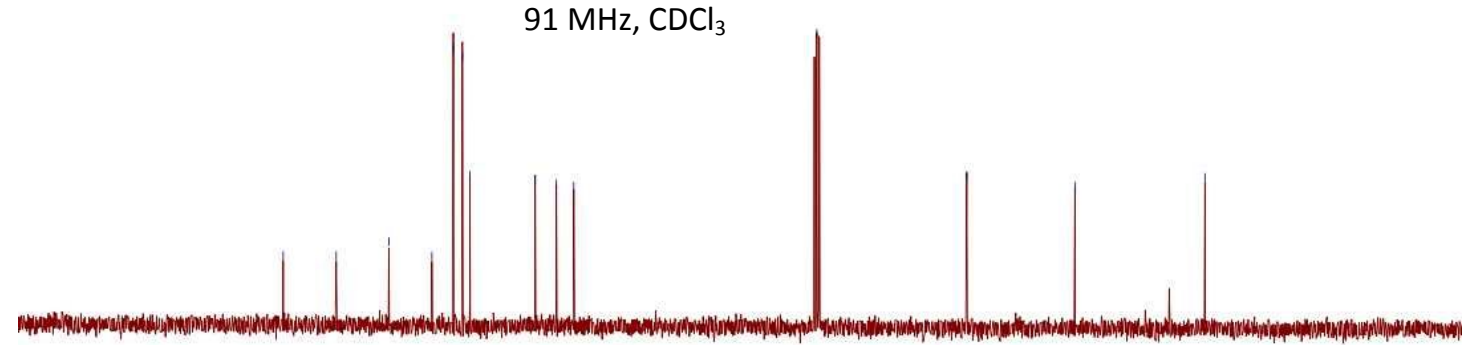

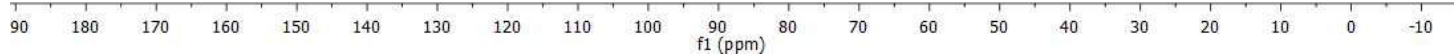




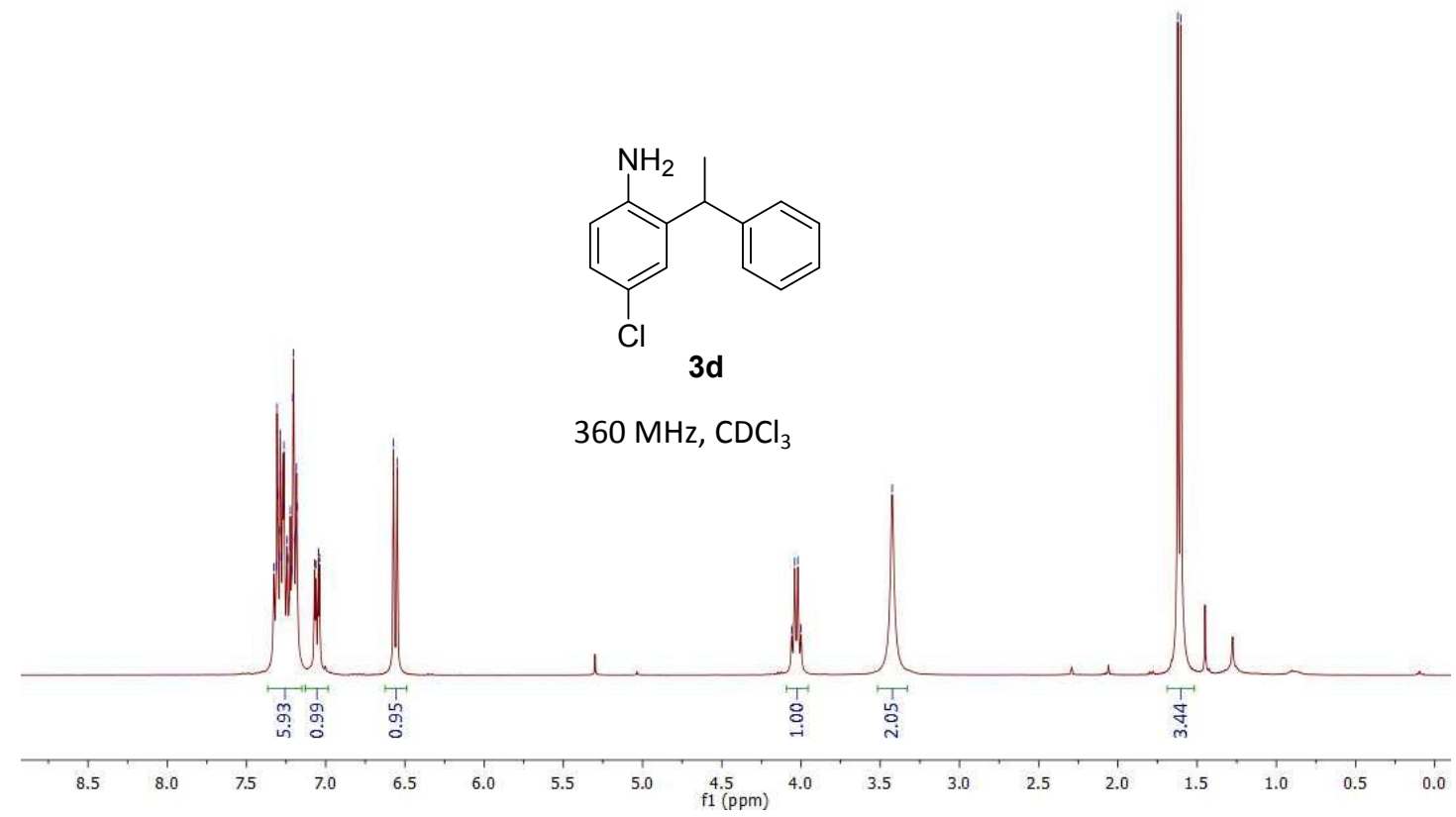

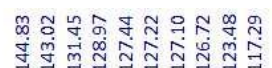

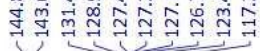

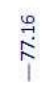

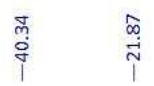

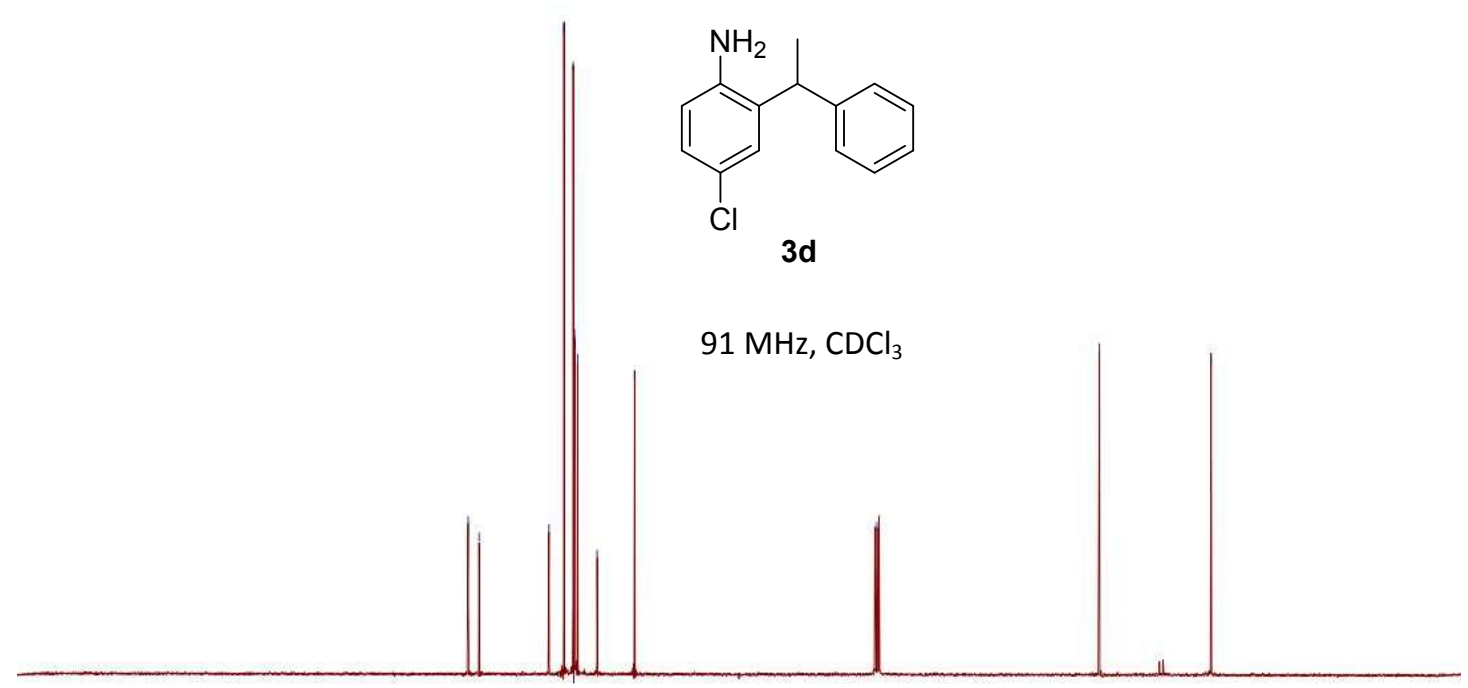

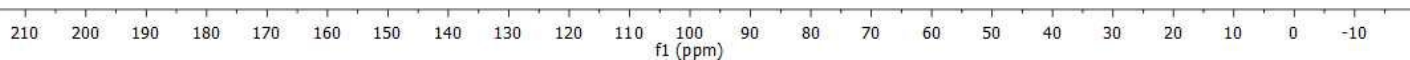




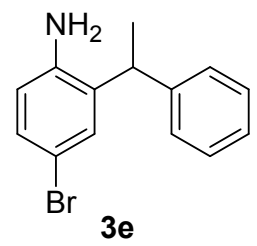

$300 \mathrm{MHz}, \mathrm{CDCl}_{3}$

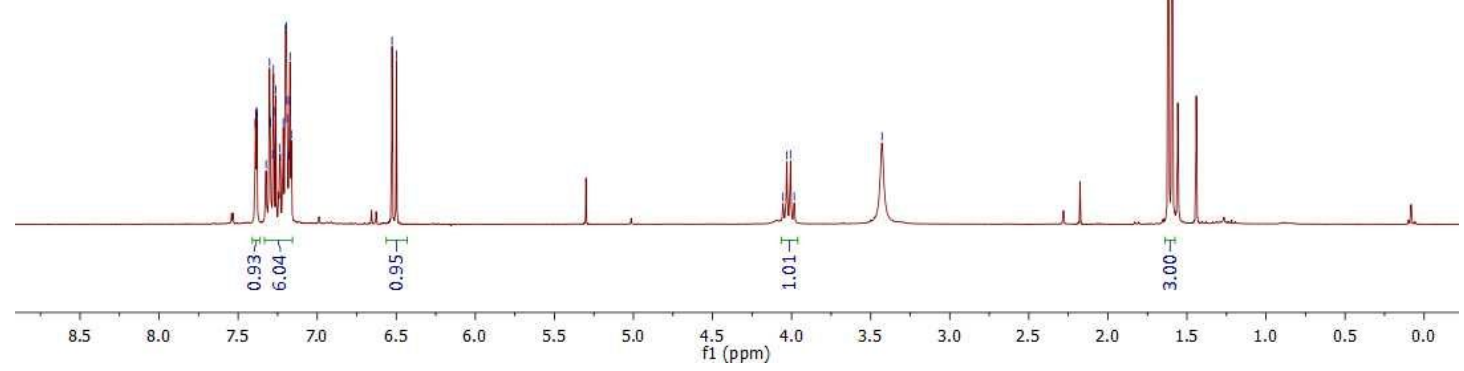

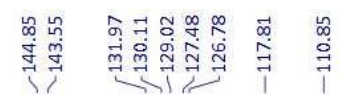

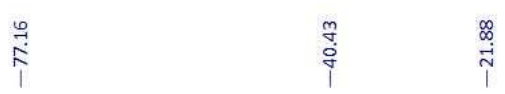<smiles>CC(c1ccccc1)c1cc(Br)ccc1N</smiles>
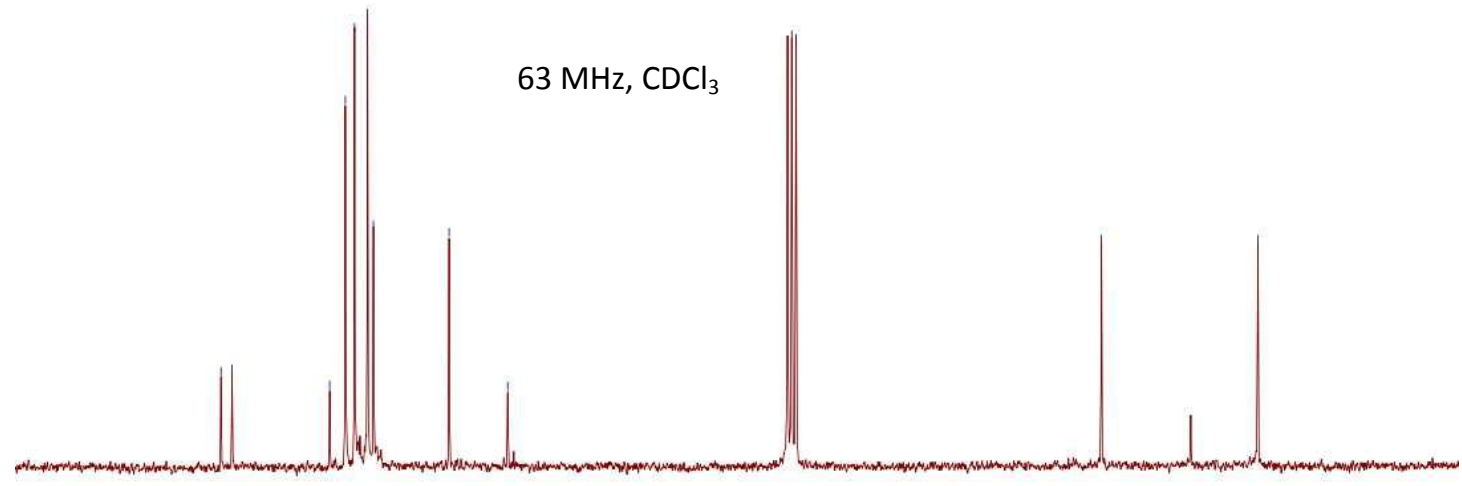

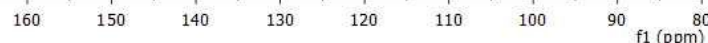




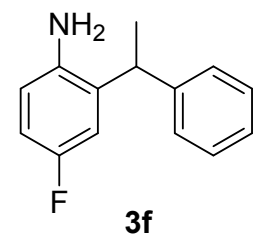

$300 \mathrm{MHz}^{\mathrm{CDCl}} \mathrm{CD}_{3}$
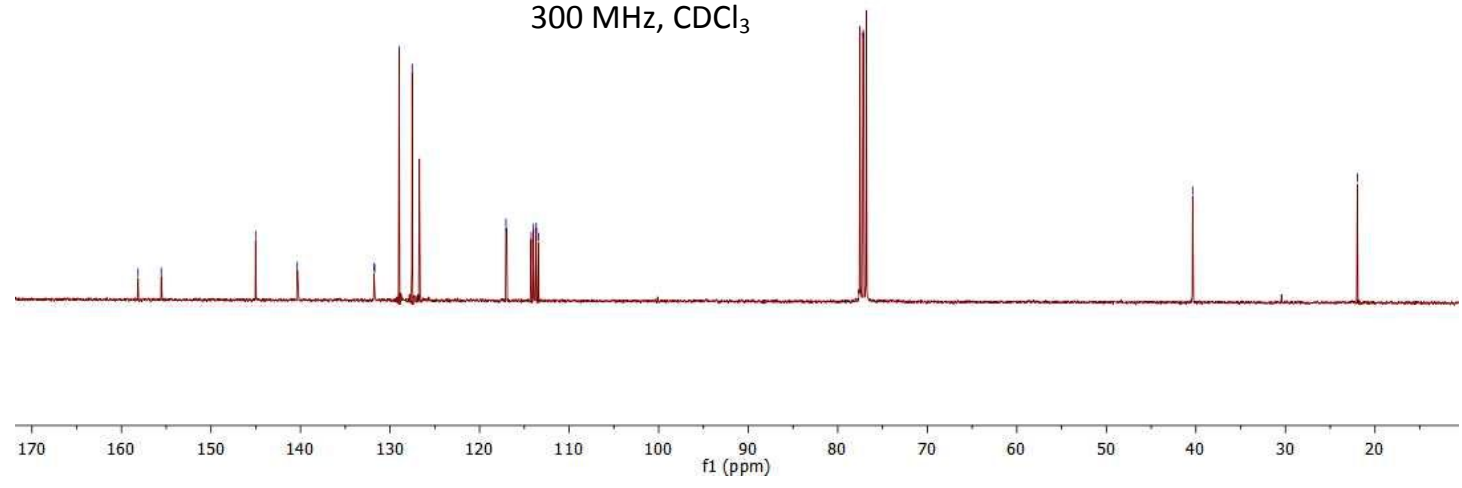

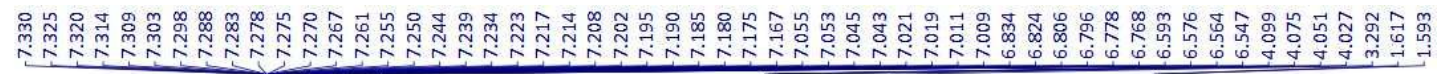

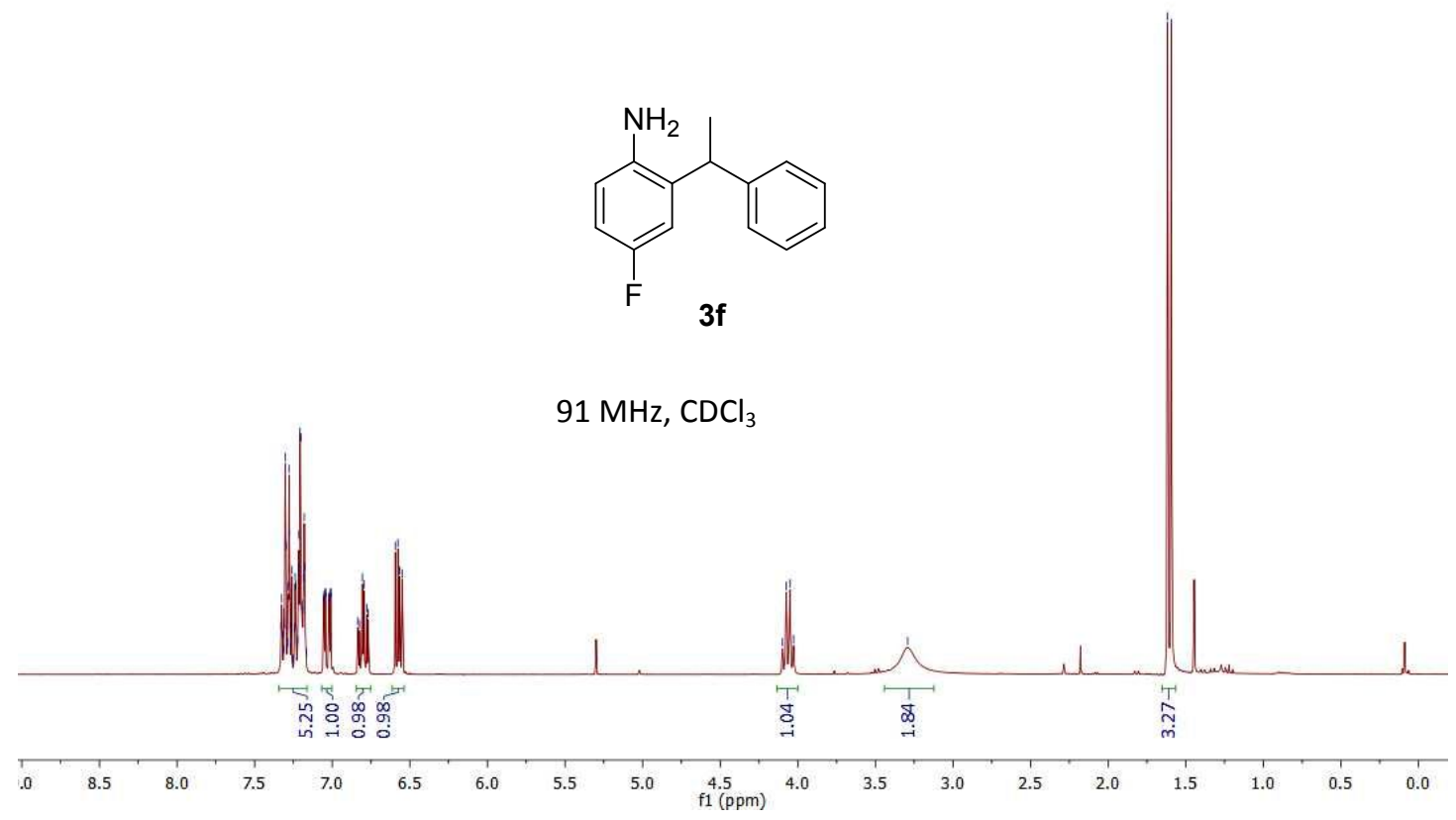




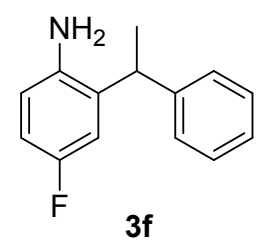

$235 \mathrm{MHz}, \mathrm{CDCl}_{3}$

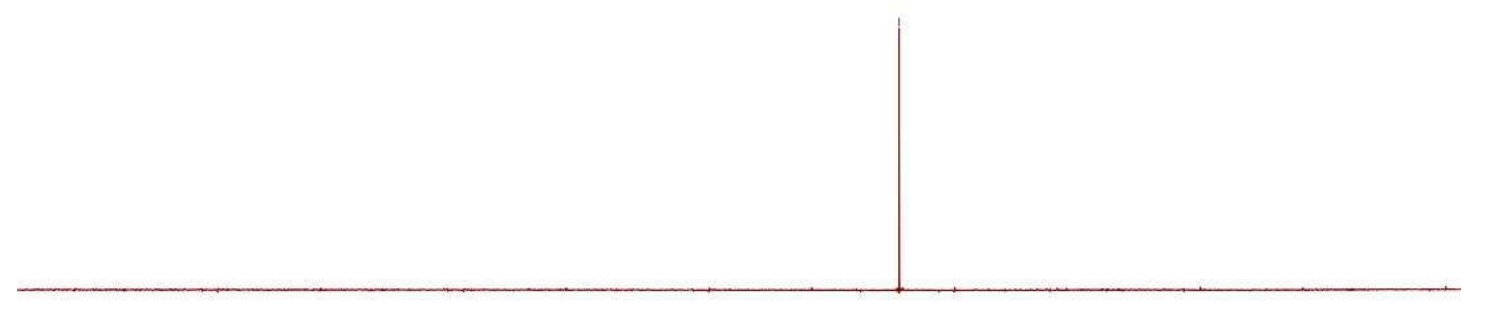

$\begin{array}{lllllllllllllllllllllllllll}1 & 10 & 10 & 0 & -10 & -20 & -30 & -40 & -50 & -60 & -70 & -80 & -90 & -100 & -110 & -120 & -130 & -140 & -150 & -160 & -170 & -180 & -190 & -200 & -210 & -220\end{array}$ 


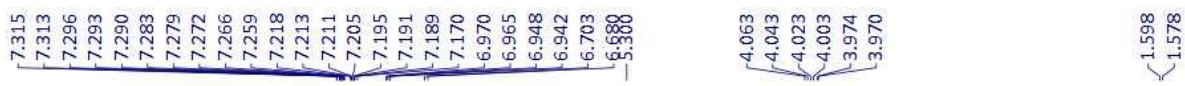

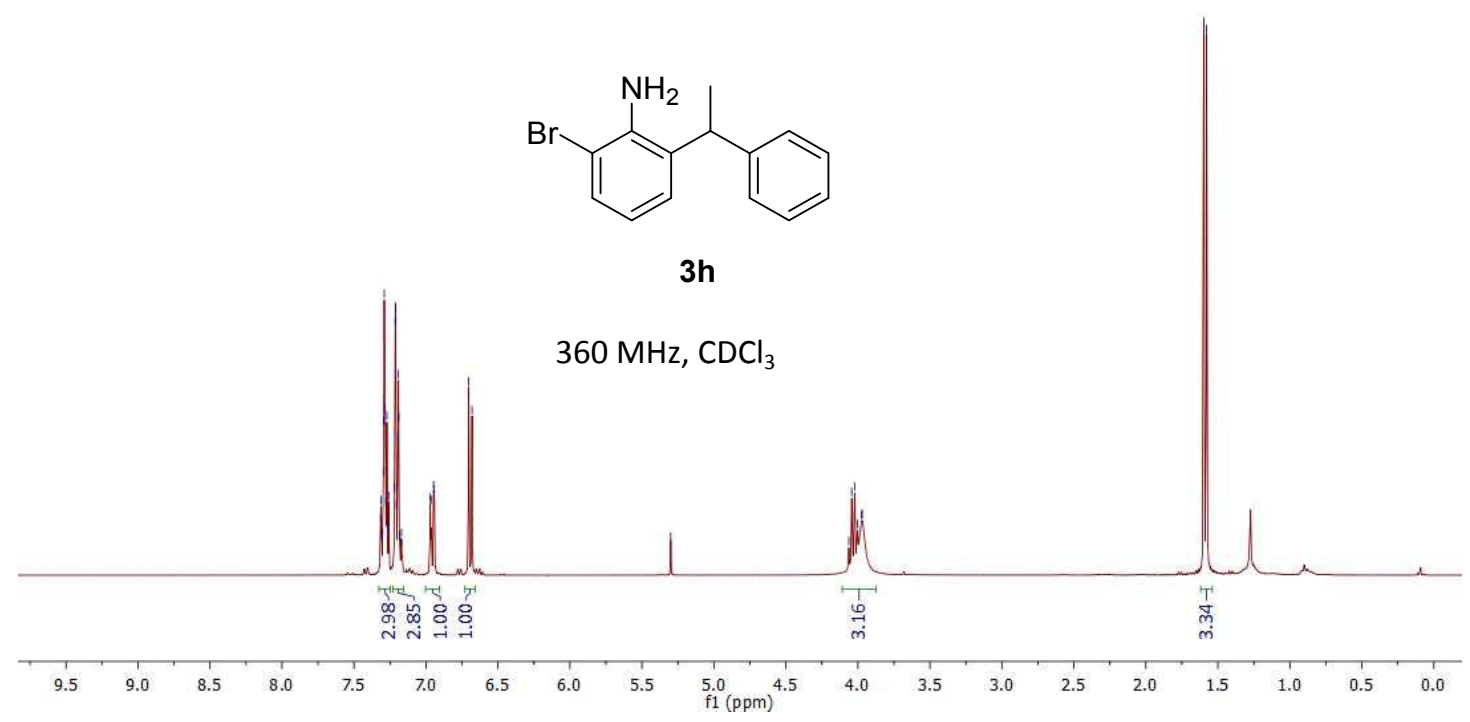

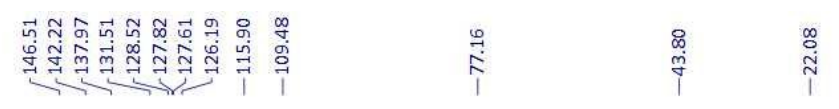

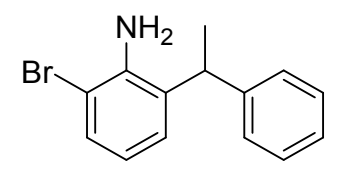

$3 h$

$63 \mathrm{MHz}, \mathrm{CDCl}_{3}$

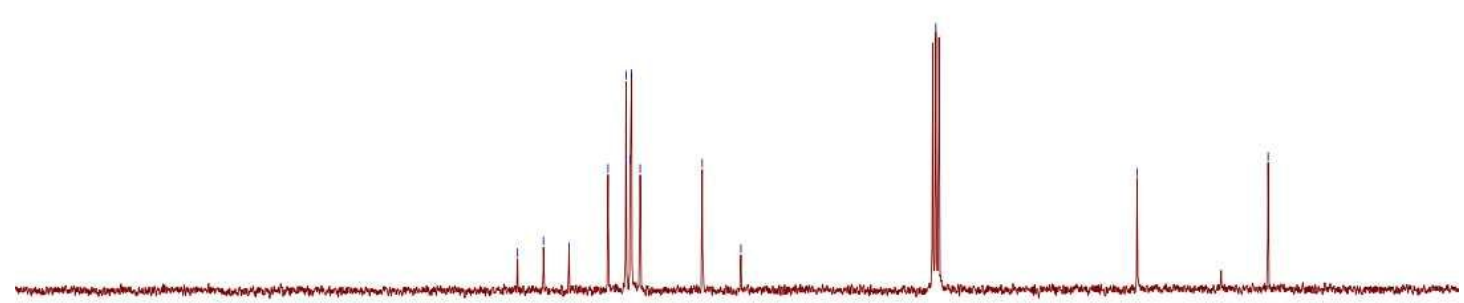

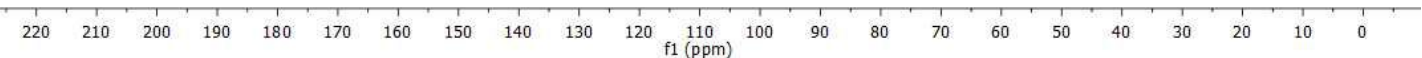




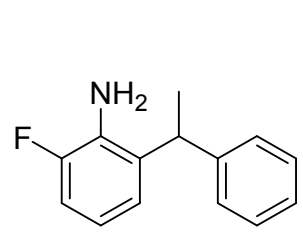

$3 \mathbf{i}$

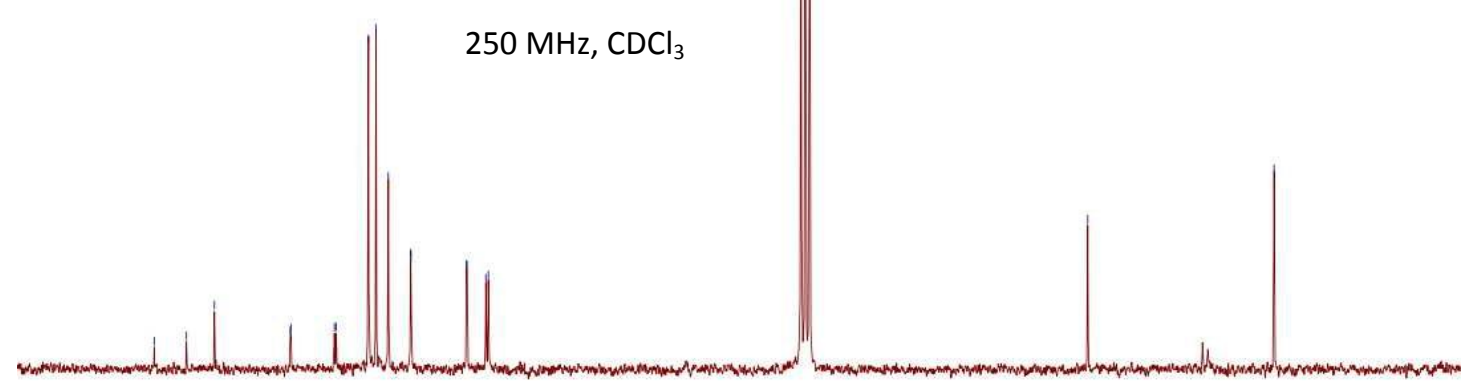

$\begin{array}{llllllll}160 & 150 & 140 & 130 & 120 & 110 & 100 & 90 \\ & & & & \end{array}$

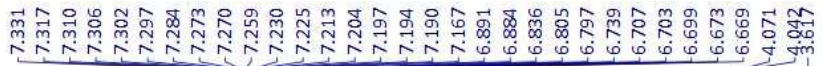

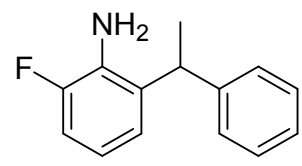

$3 \mathbf{i}$

$63 \mathrm{MHz}, \mathrm{CDCl}_{3}$

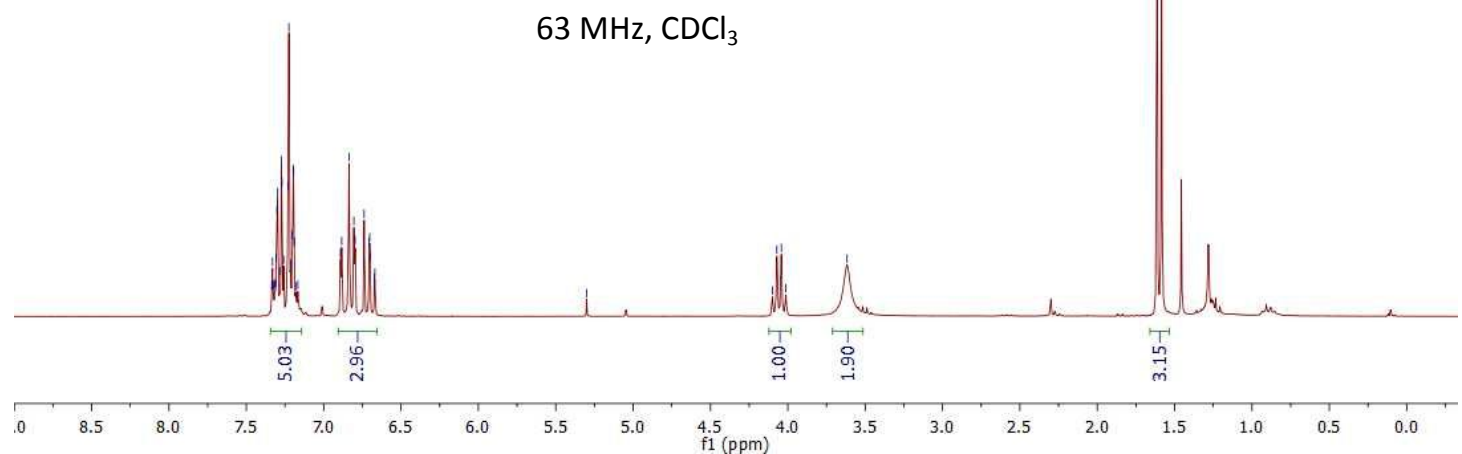




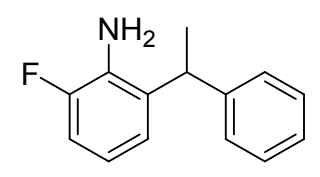

$3 \mathbf{i}$

$235 \mathrm{MHz}, \mathrm{CDCl}_{3}$

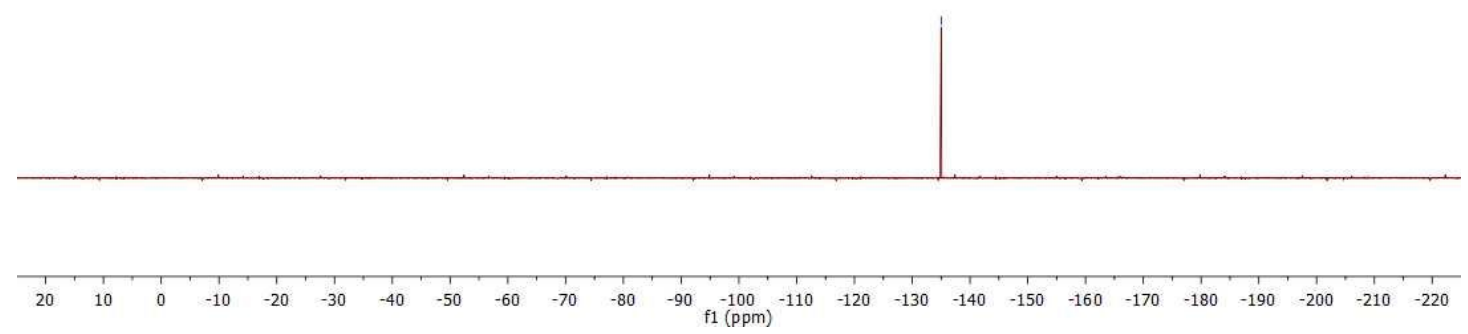




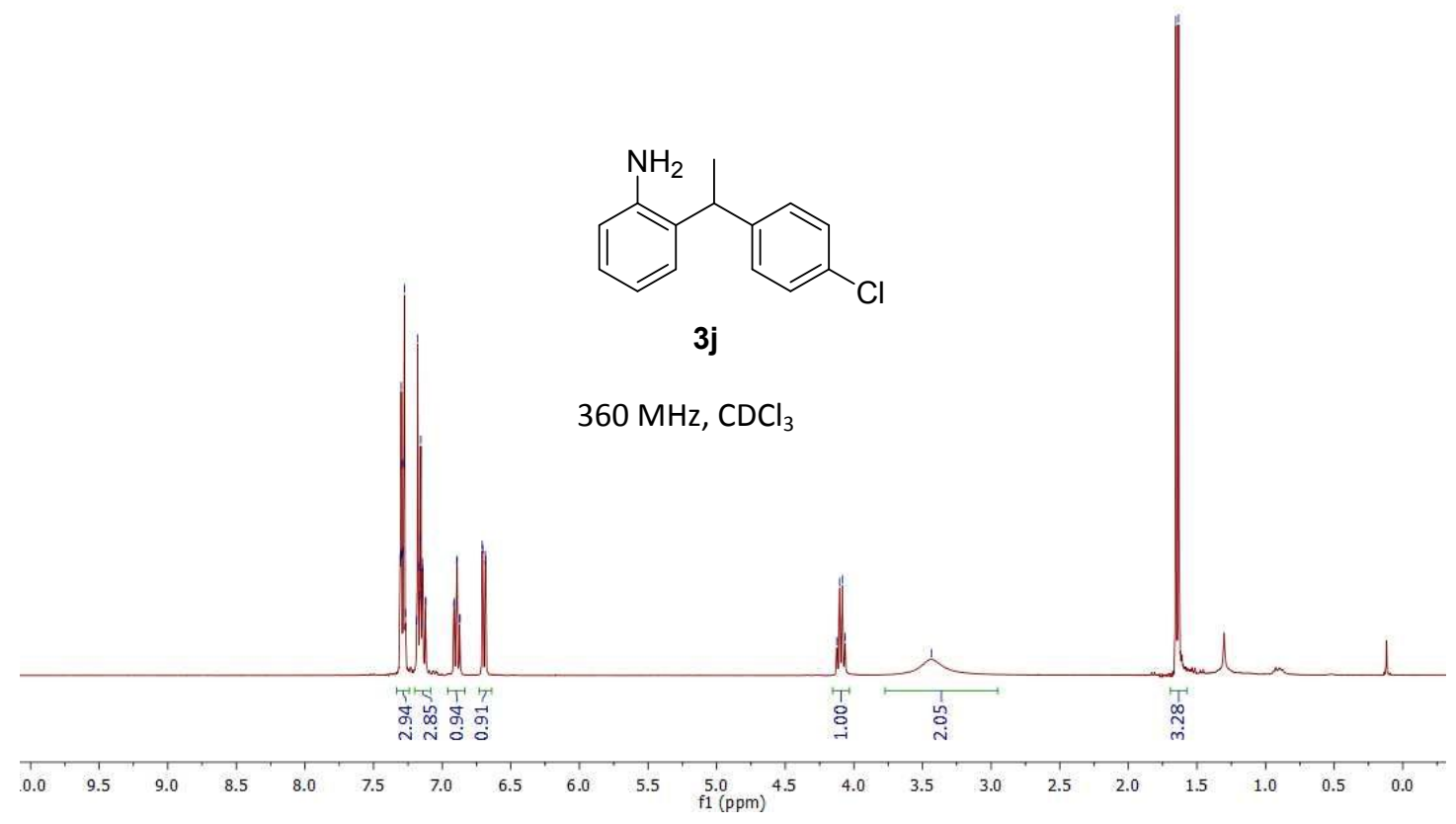

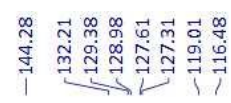

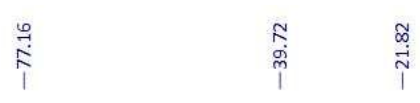<smiles>CC(c1ccc(Cl)cc1)c1ccccc1N</smiles>

3j

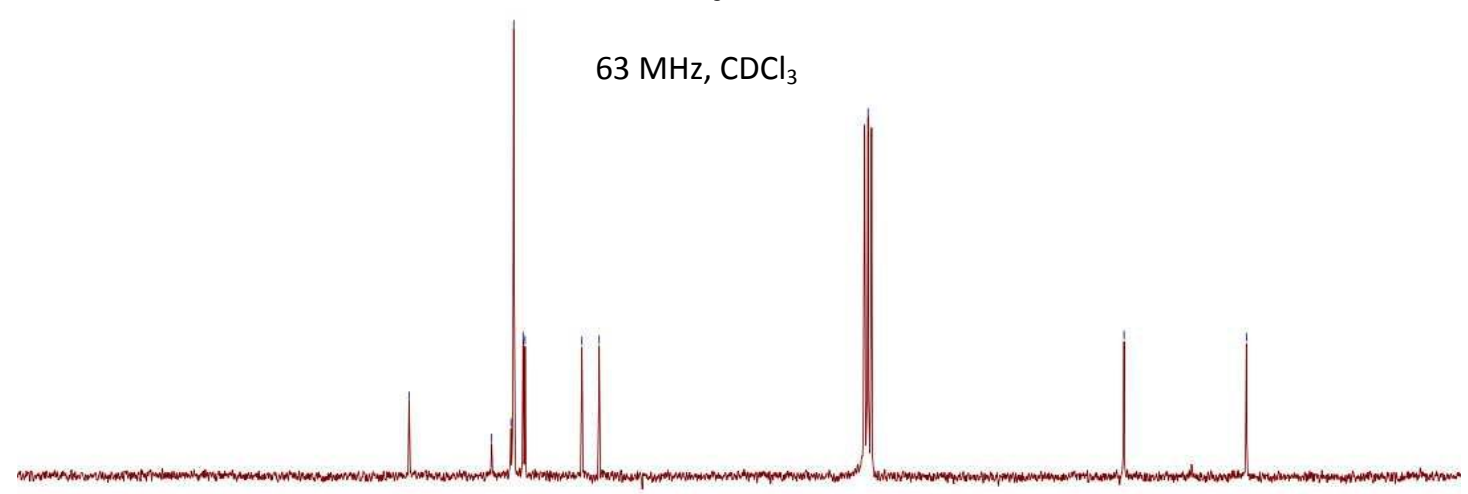

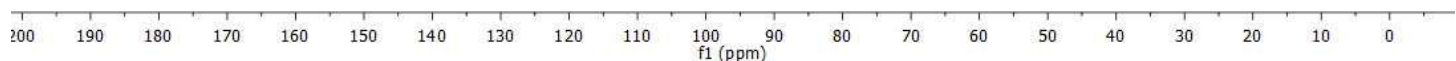



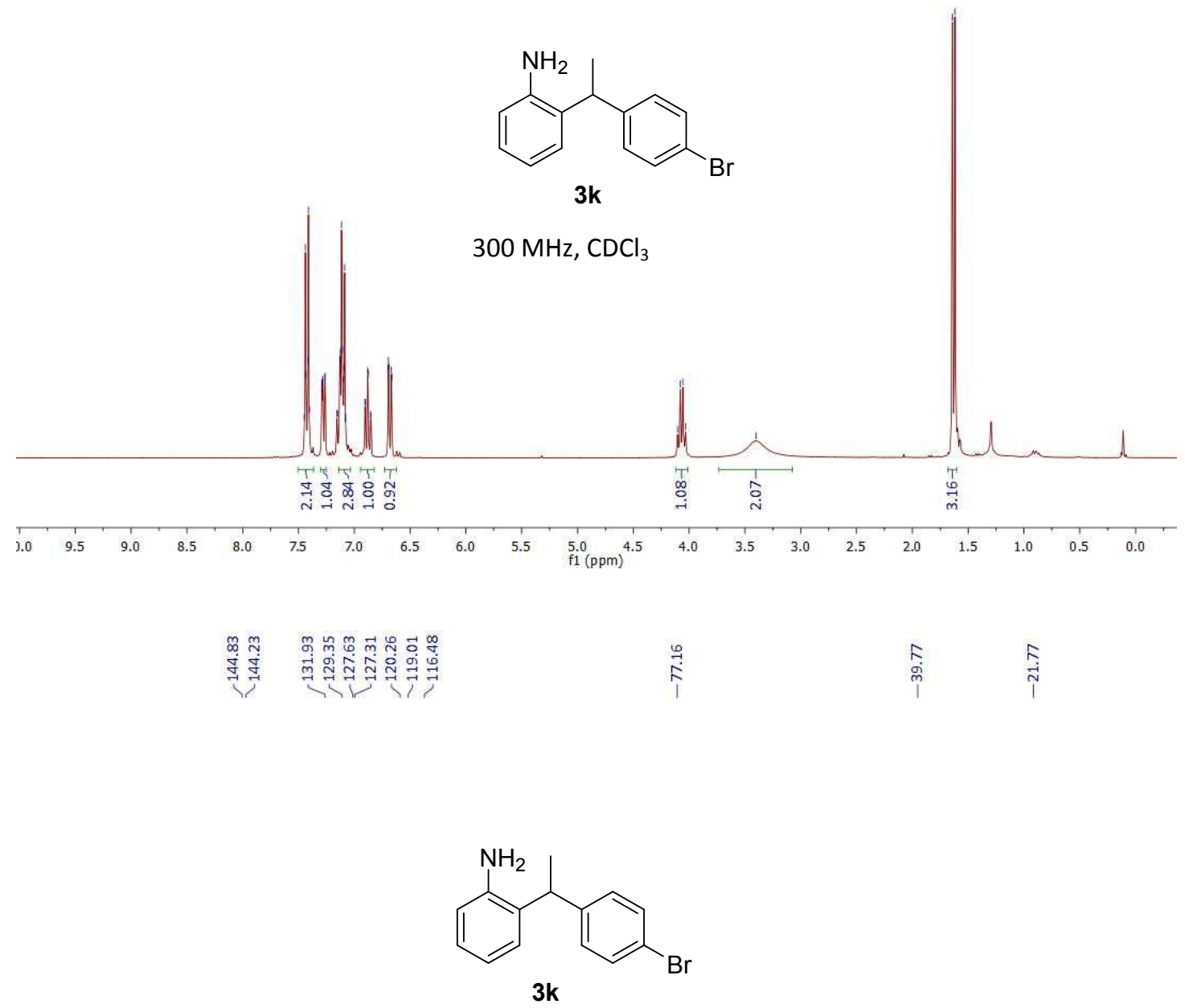

$63 \mathrm{MHz}, \mathrm{CDCl}_{3}$

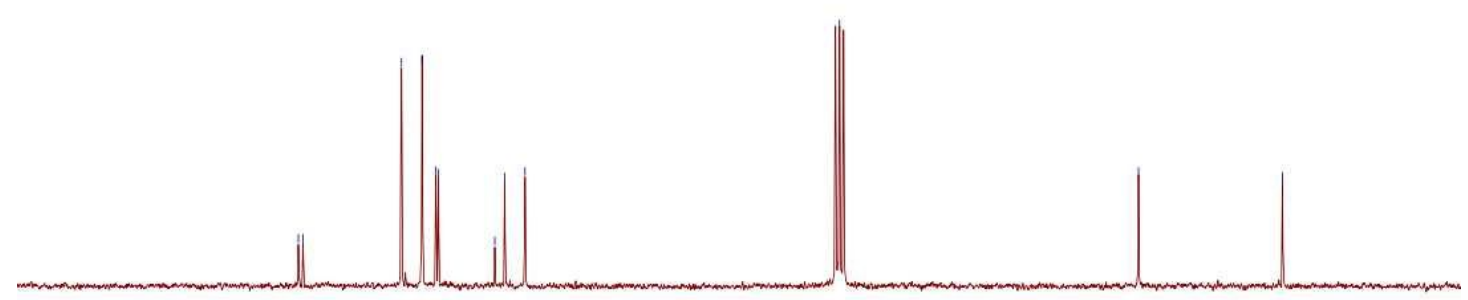

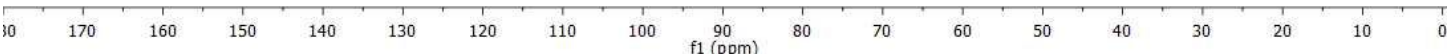


<smiles>CC(c1ccc(F)cc1)c1ccccc1N</smiles>

3I

$300 \mathrm{MHz}, \mathrm{CDCl}_{3}$

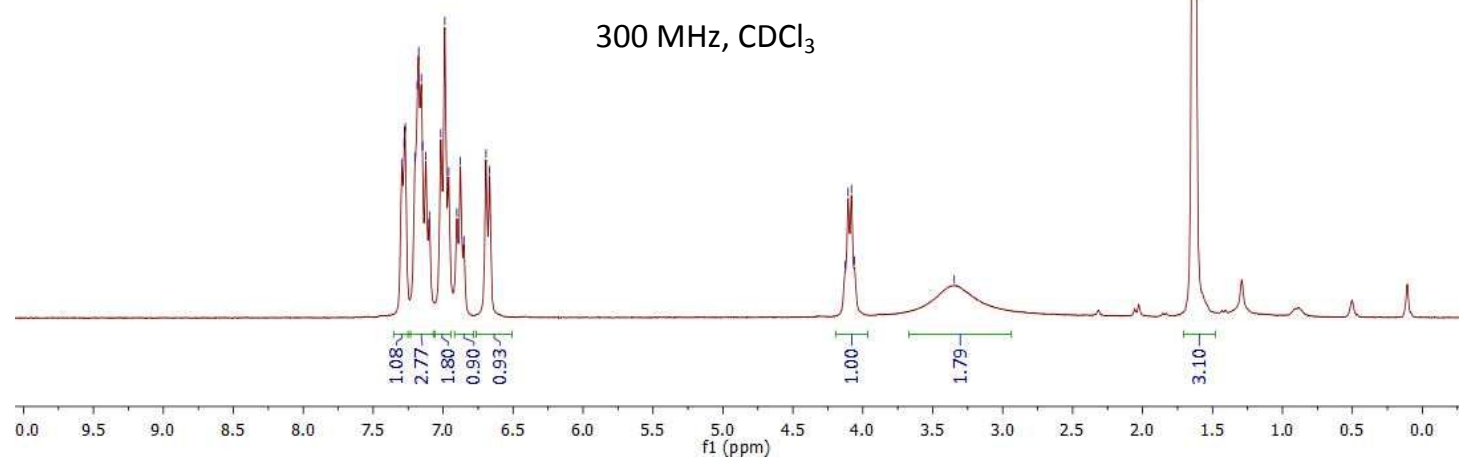

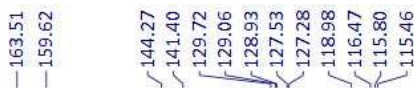

$\stackrel{\infty}{\stackrel{1}{\pi}}$

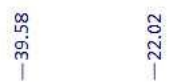<smiles>CC(c1ccc(F)cc1)c1ccccc1N</smiles>

3I

$63 \mathrm{MHz}, \mathrm{CDCl}_{3}$

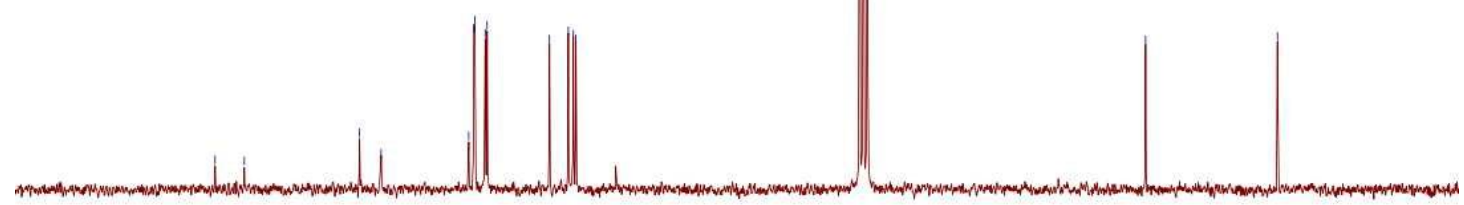

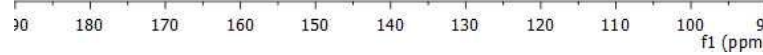




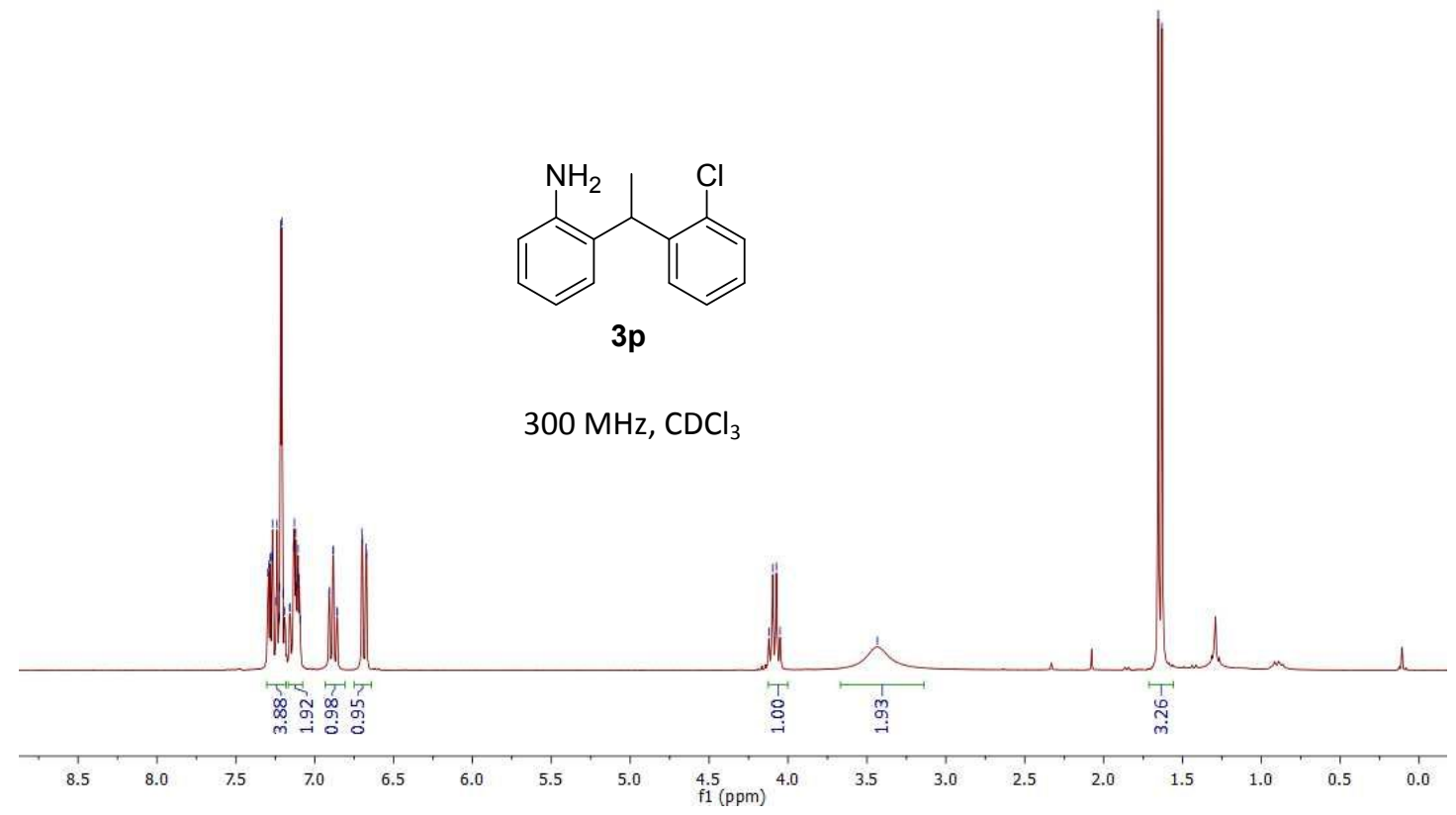

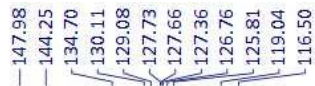

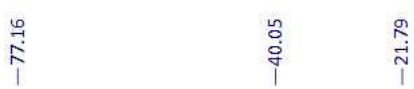<smiles>CC(c1ccccc1N)c1ccccc1Cl</smiles>

$3 p$

$63 \mathrm{MHz}, \mathrm{CDCl}_{3}$

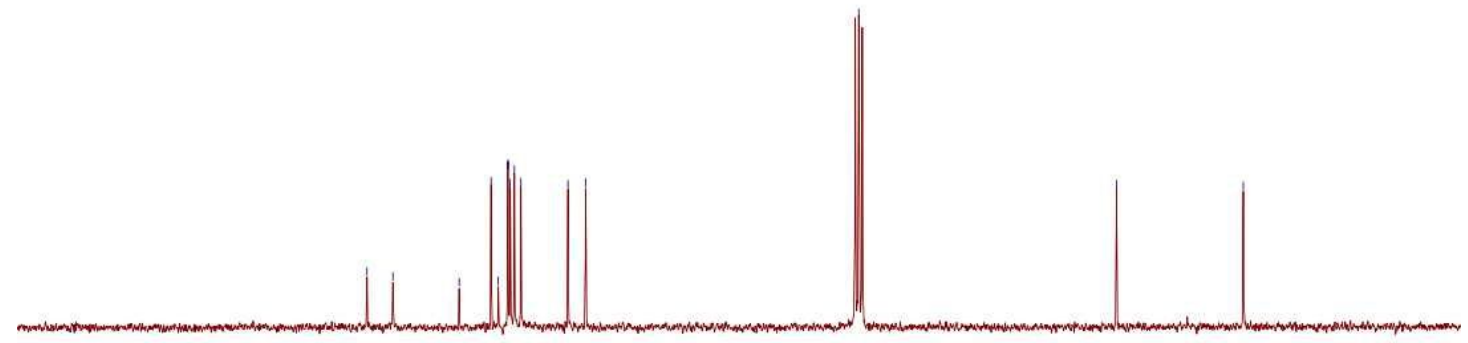

$\begin{array}{llllllllll}190 & 180 & 170 & 160 & 150 & 140 & 130 & 120 & 110 & 100 \\ \mathrm{f} 1(\mathrm{ppm})\end{array}$ 


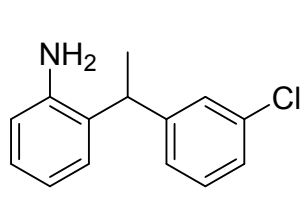

$3 q$

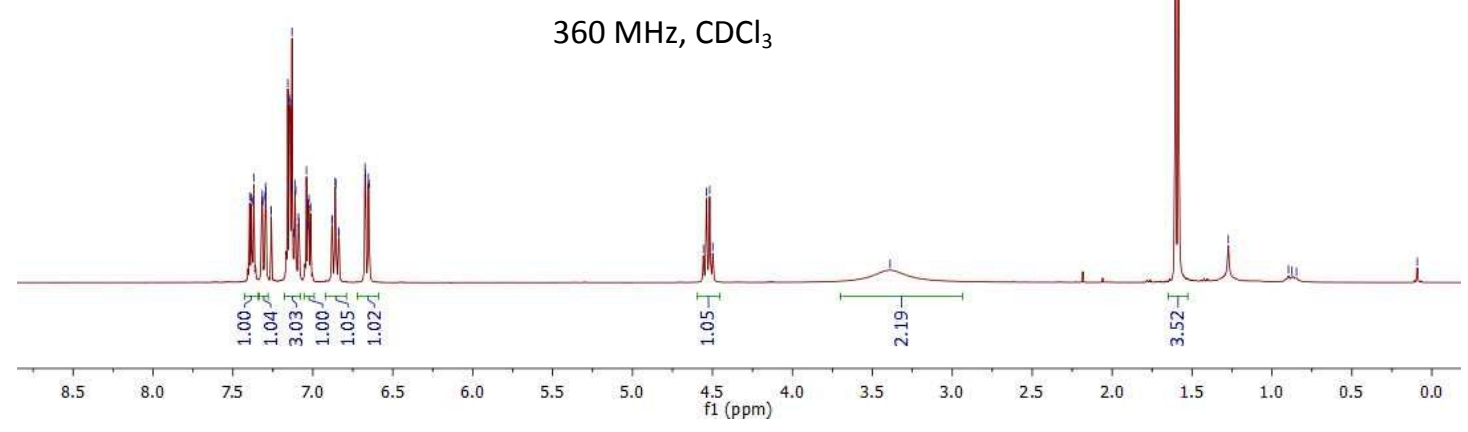

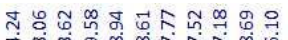

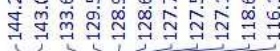

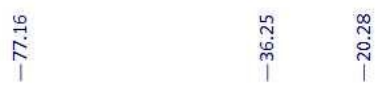

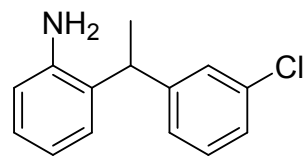

$3 q$

$63 \mathrm{MHz}^{\mathrm{CDCl}_{3}}$

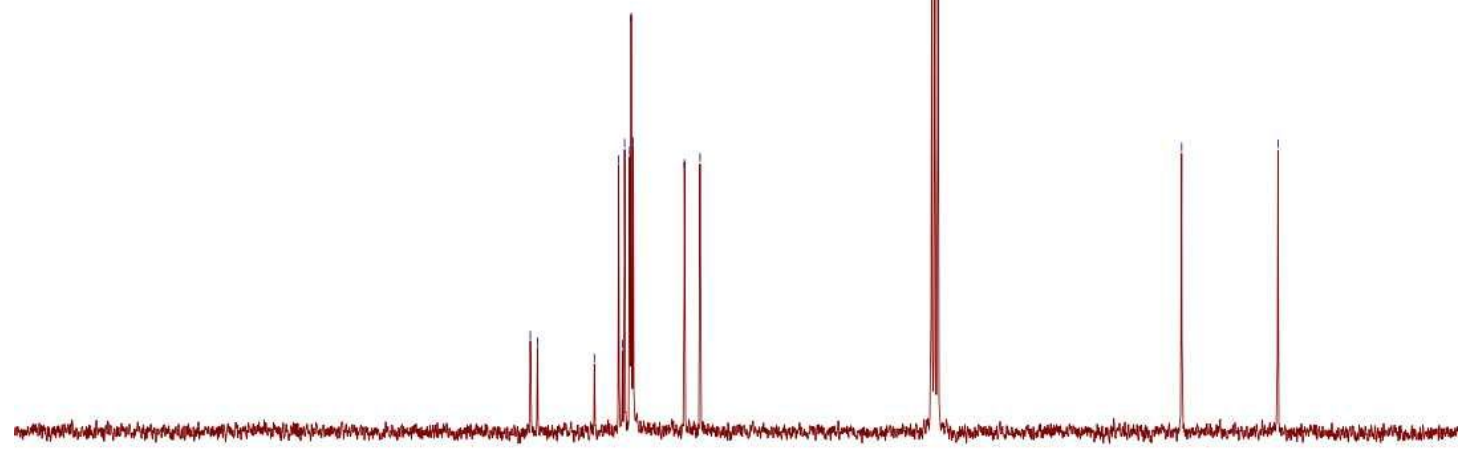

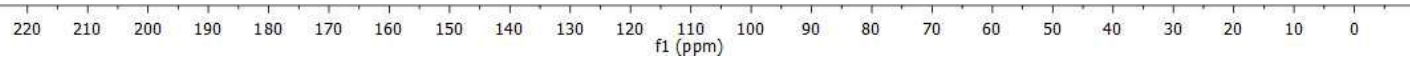




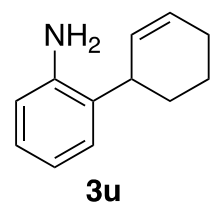

$360 \mathrm{MHz}, \mathrm{CDCl}_{3}$
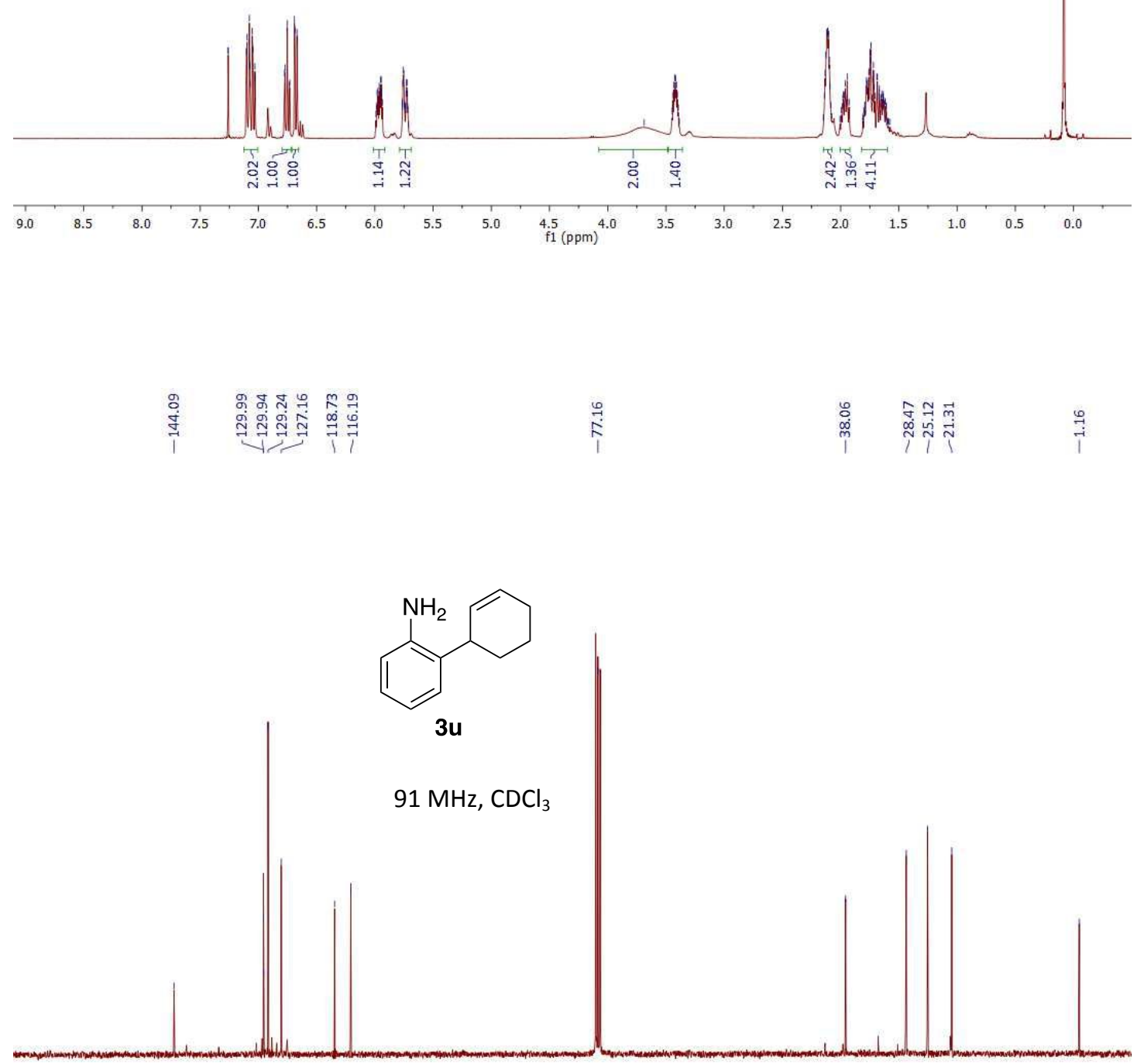


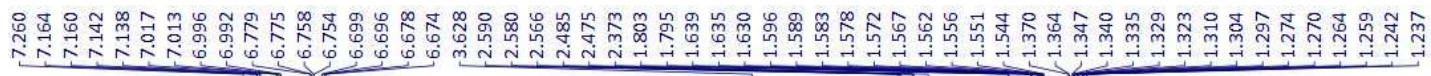<smiles>Nc1ccccc1C1CC2CCC1C2</smiles>

3v

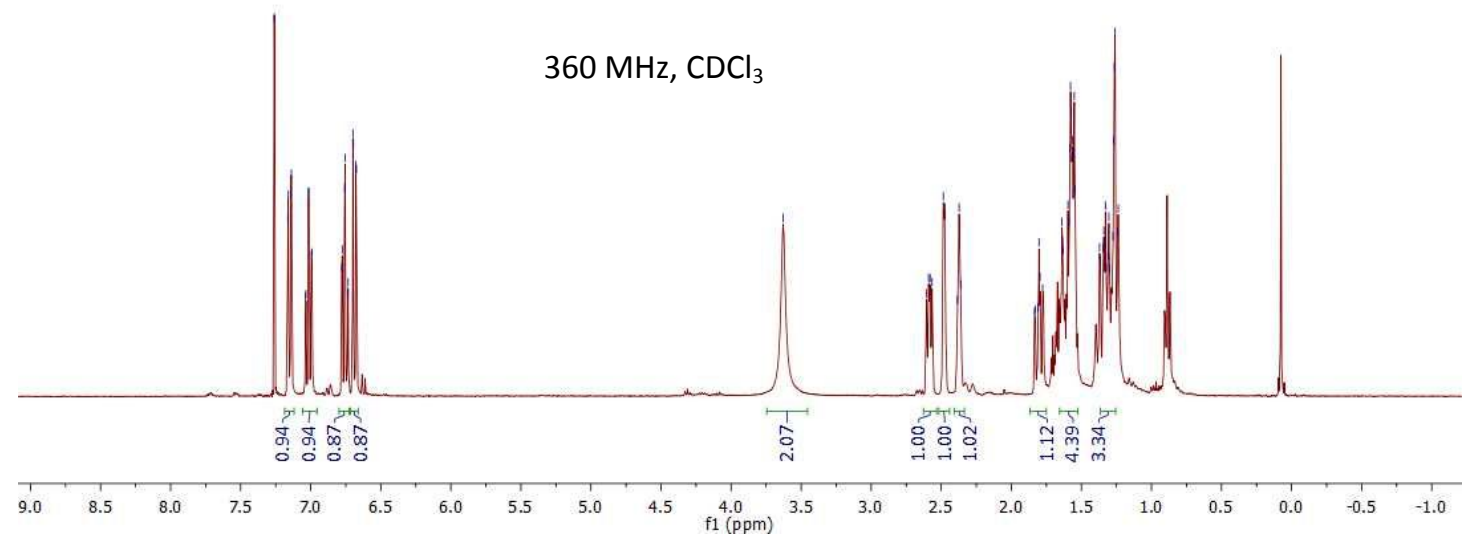

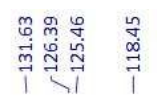<smiles>Nc1ccccc1C1CC2CCC1C2</smiles>

$91 \mathrm{MHz}, \mathrm{CDCl}_{3}$

$\begin{array}{lllllllll}170 & 160 & 150 & 140 & 130 & 120 & 110 & 100 & 90 \\ & 1(\mathrm{ppm}) & 80\end{array}$

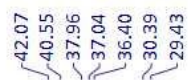

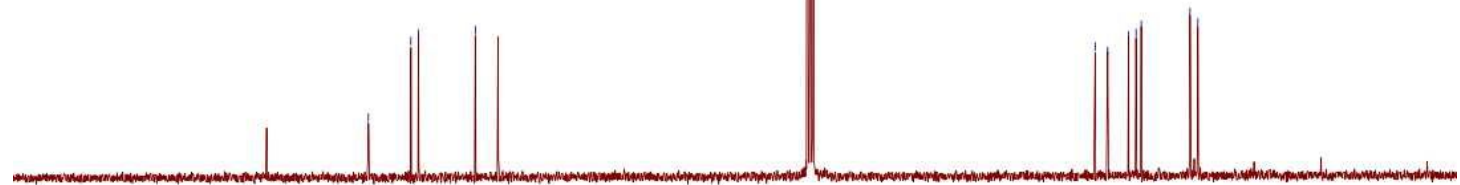



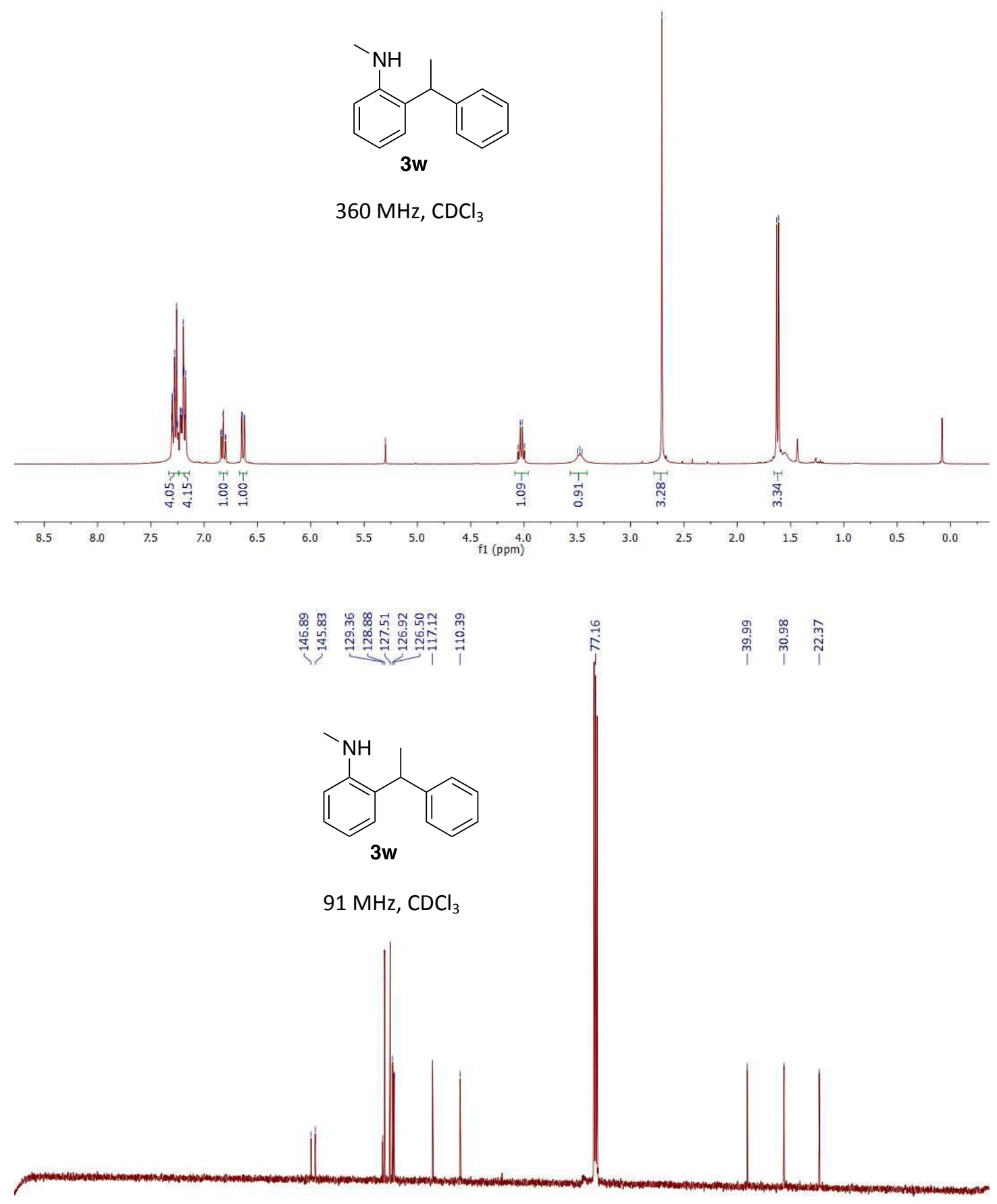

$\begin{array}{lllllllllllllllllllllllllll}210 & 200 & 190 & 180 & 170 & 160 & 150 & 140 & 130 & 120 & 110 & 100 & 100 & 80 & 70 & 60 & 50 & 40 & 30 & 20 & 10 & 0 & -10\end{array}$ 


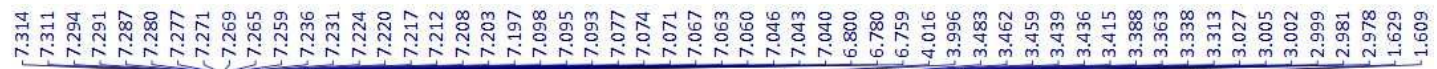

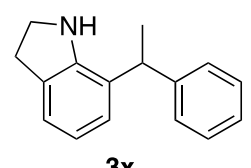

$360 \mathrm{MHz}, \mathrm{CDCl}_{3}$
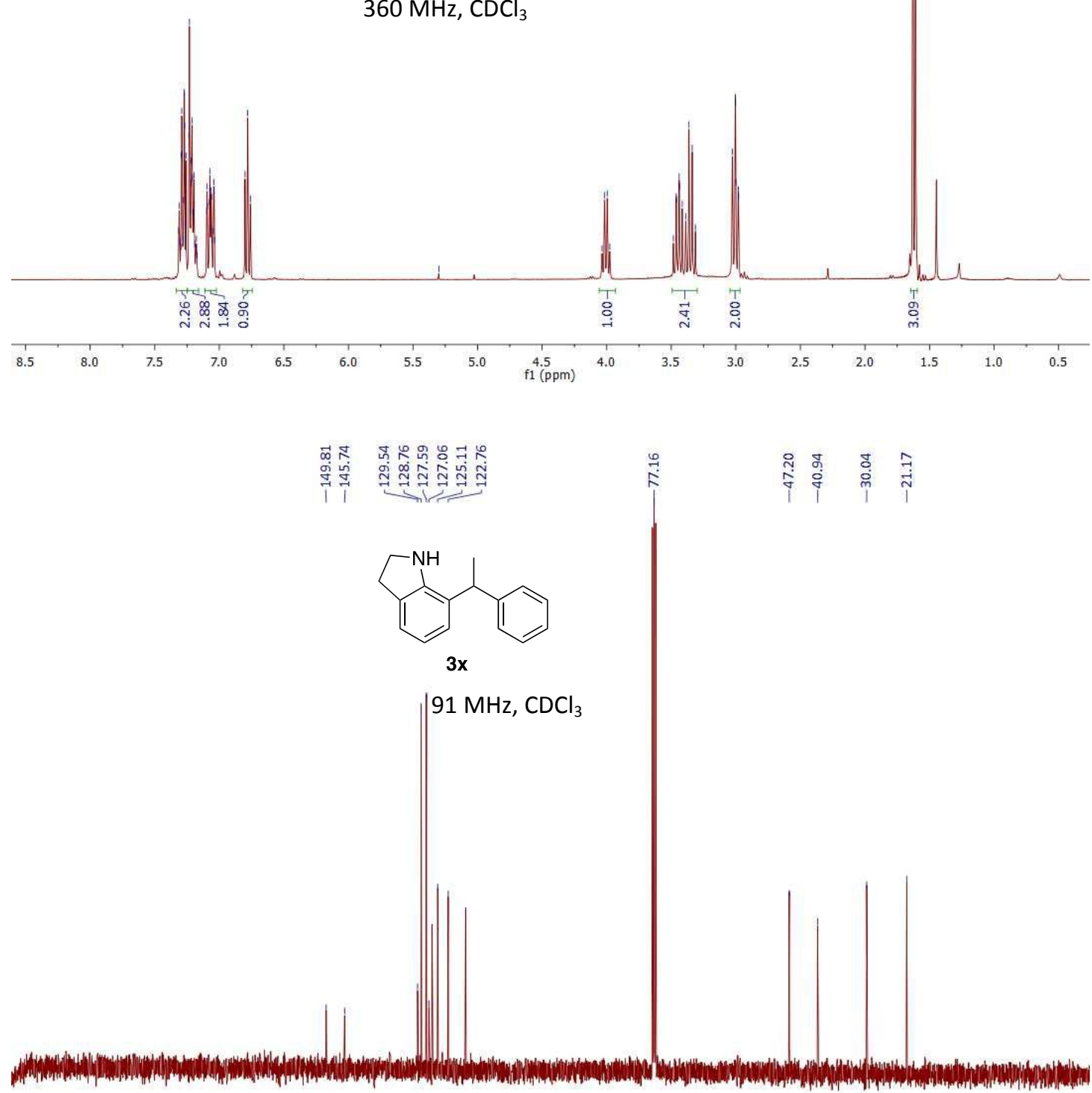

$\begin{array}{lllllllllllllllllllllllllll}210 & 200 & 190 & 180 & 170 & 160 & 150 & 140 & 130 & 120 & 110 & \begin{array}{c}100 \\ \mathrm{f} 1(\mathrm{ppm})\end{array} & 90 & 80 & 70 & 60 & 50 & 40 & 30 & 20 & 10 & 0 & -10\end{array}$ 


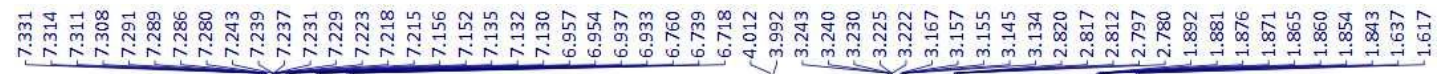

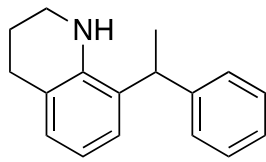

3y

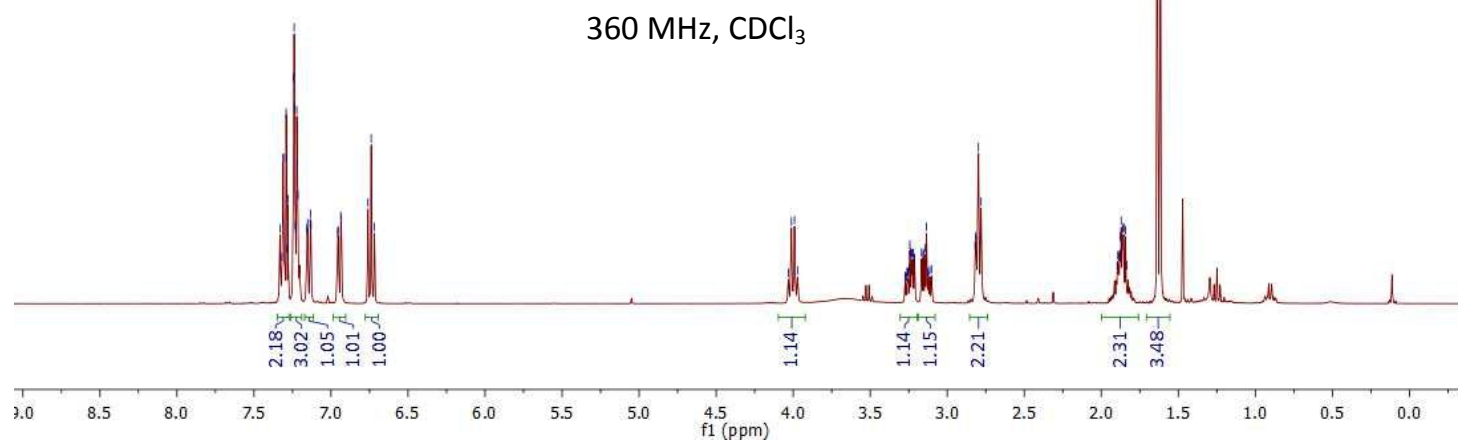

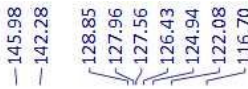

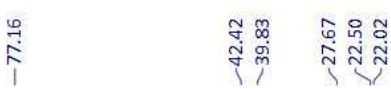

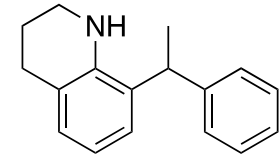

3y

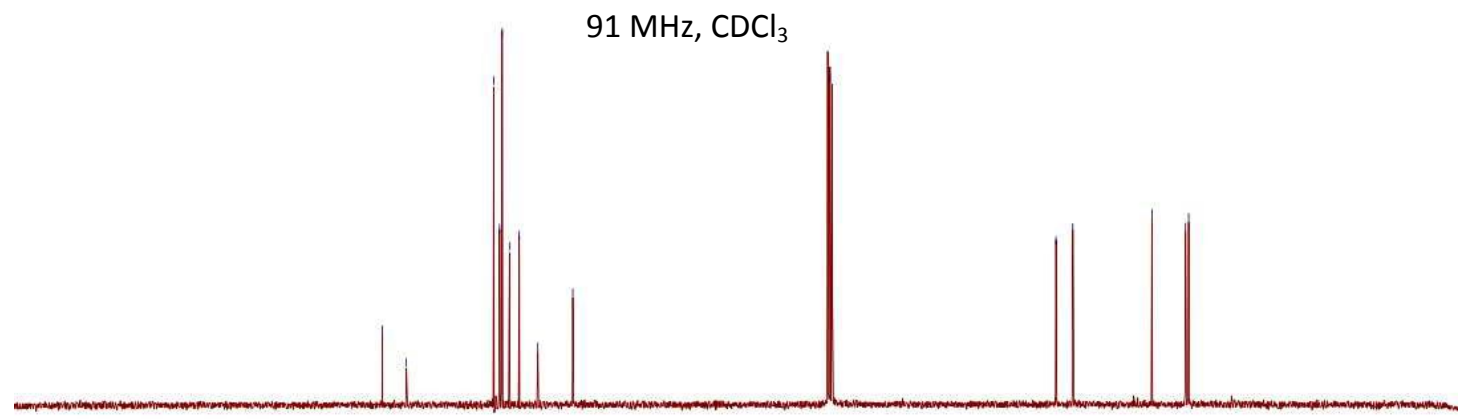

$\begin{array}{llllllllllllllllllllllllllllll}200 & 190 & 180 & 170 & 160 & 150 & 140 & 130 & 120 & 110 & 100 & 90 & 80 & 70 & 60 & 50 & 40 & 30 & 20 & 10 & 0 & -10\end{array}$ 

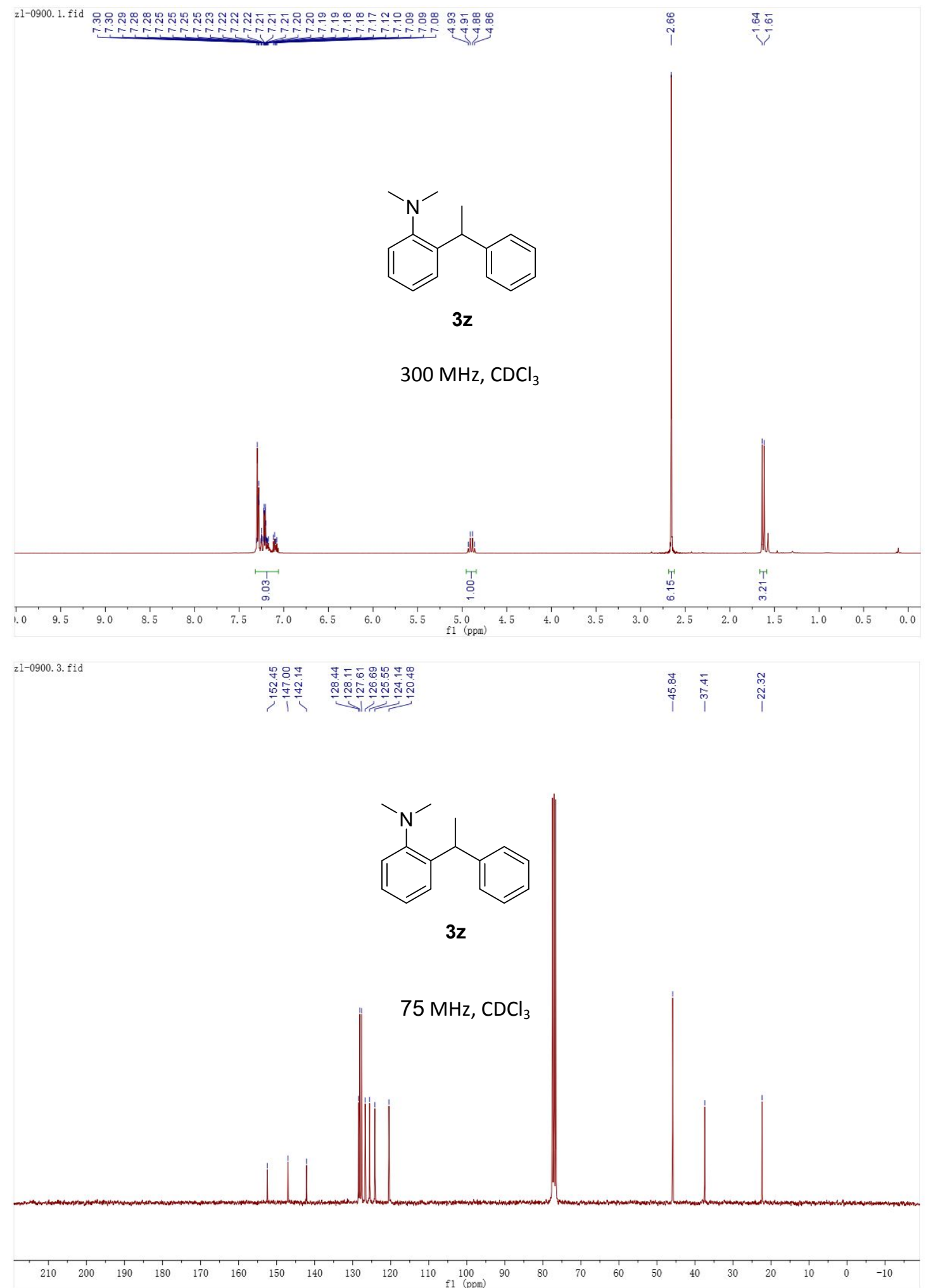


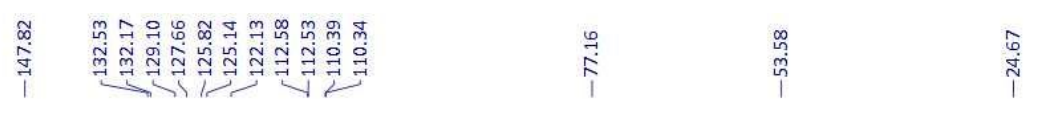

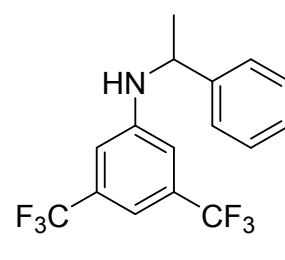

4b
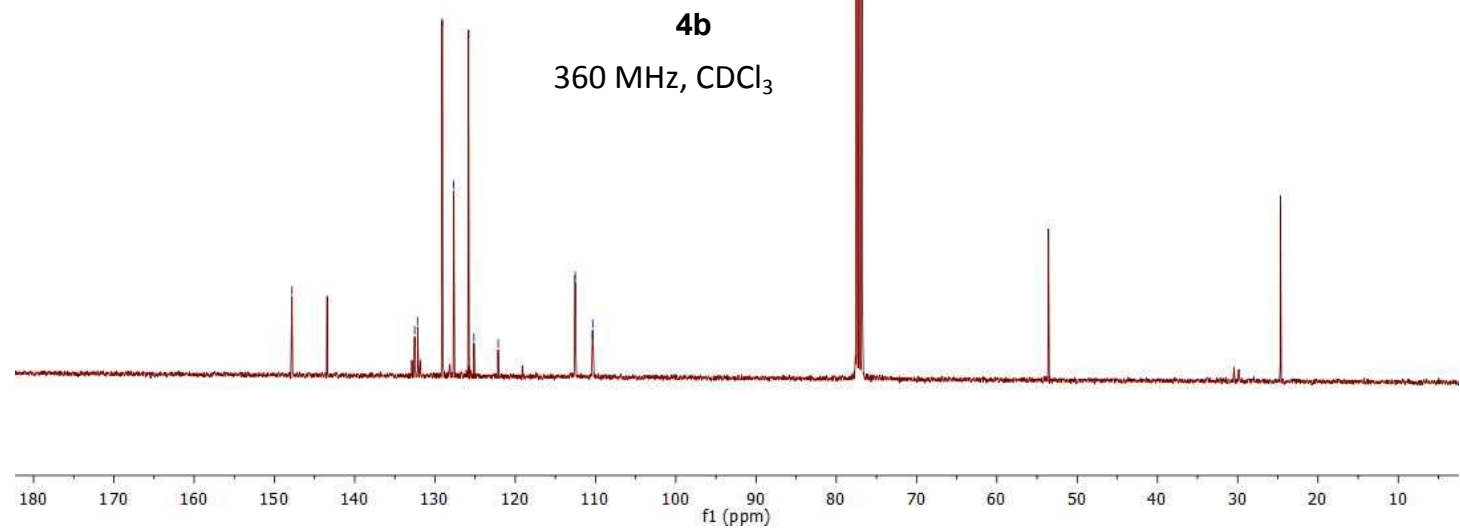

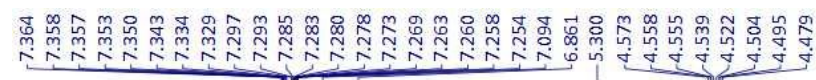

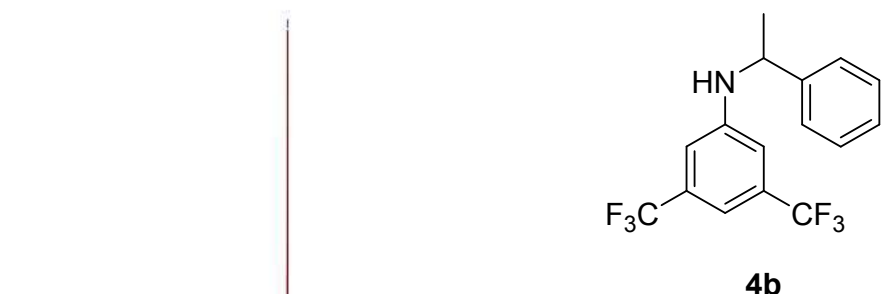

$91 \mathrm{MHz}^{\mathrm{CDCl}}{ }_{3}$

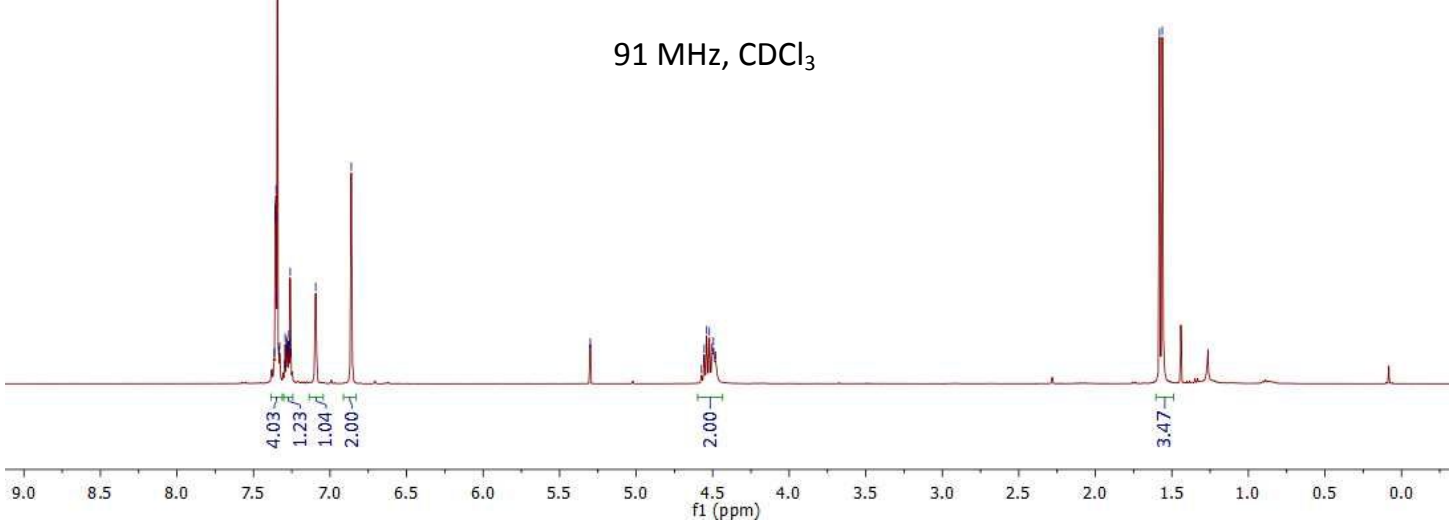




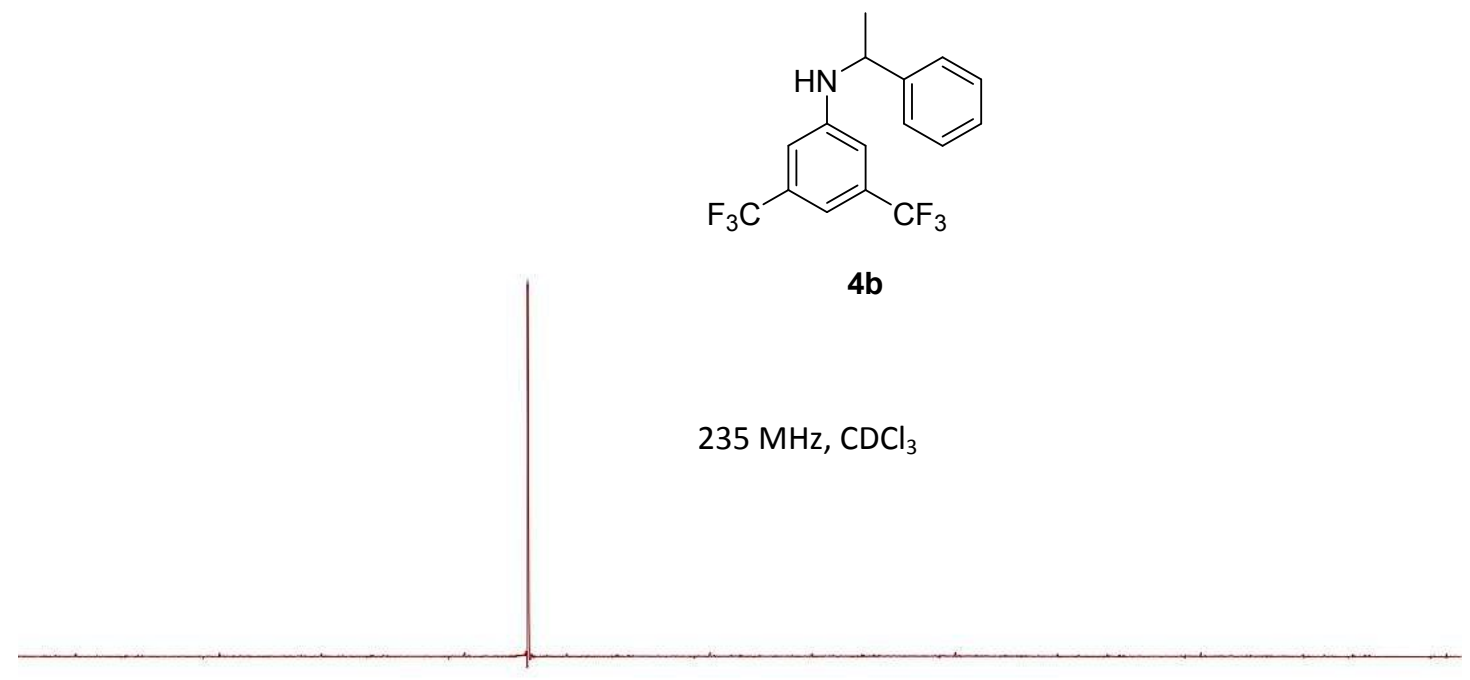

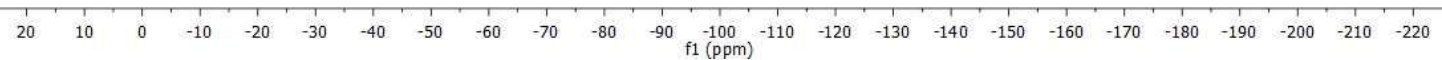



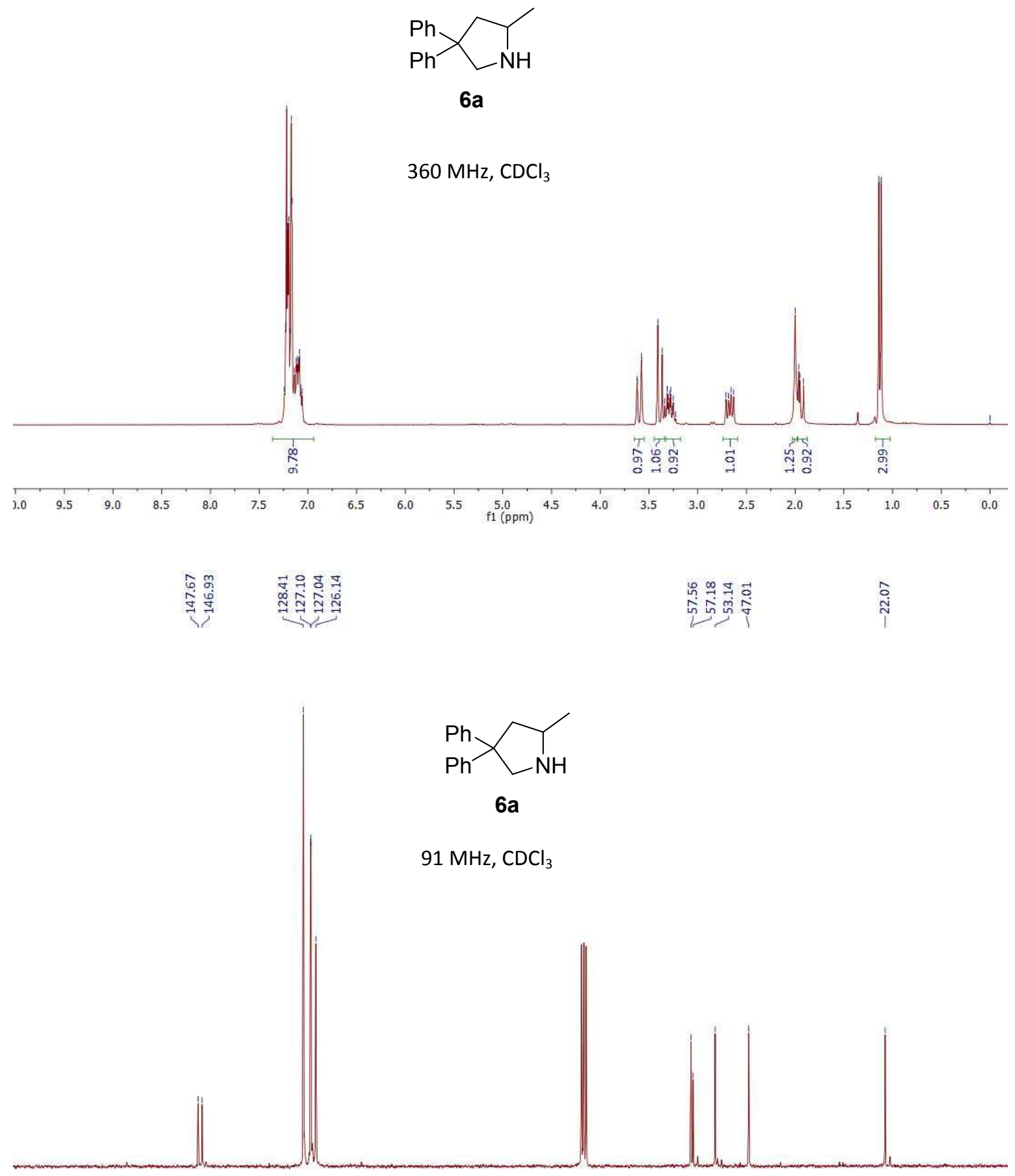

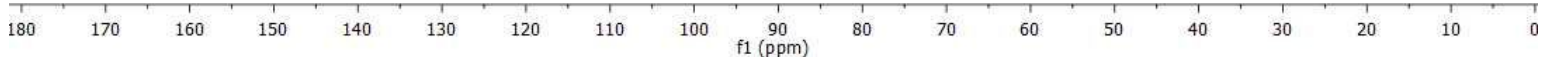




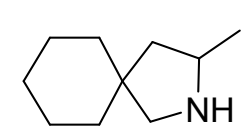

6b

$360 \mathrm{MHz}, \mathrm{CDCl}_{3}$

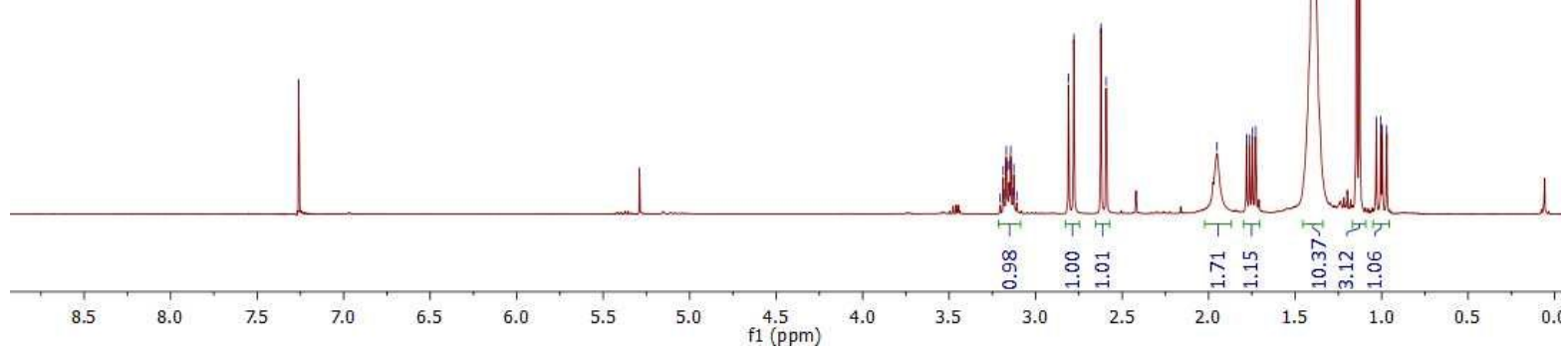

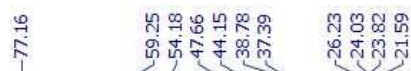

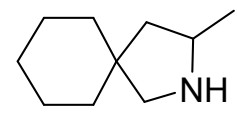

6b

$91 \mathrm{MHz}, \mathrm{CDCl}_{3}$

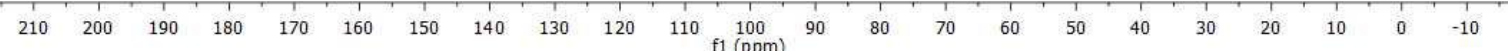




\section{is}

$\stackrel{8}{i}$

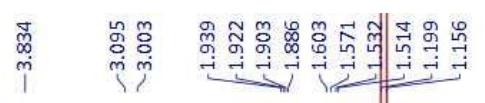

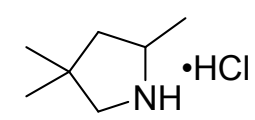

6c

$360 \mathrm{MHz}, \mathrm{CDCl}_{3}$

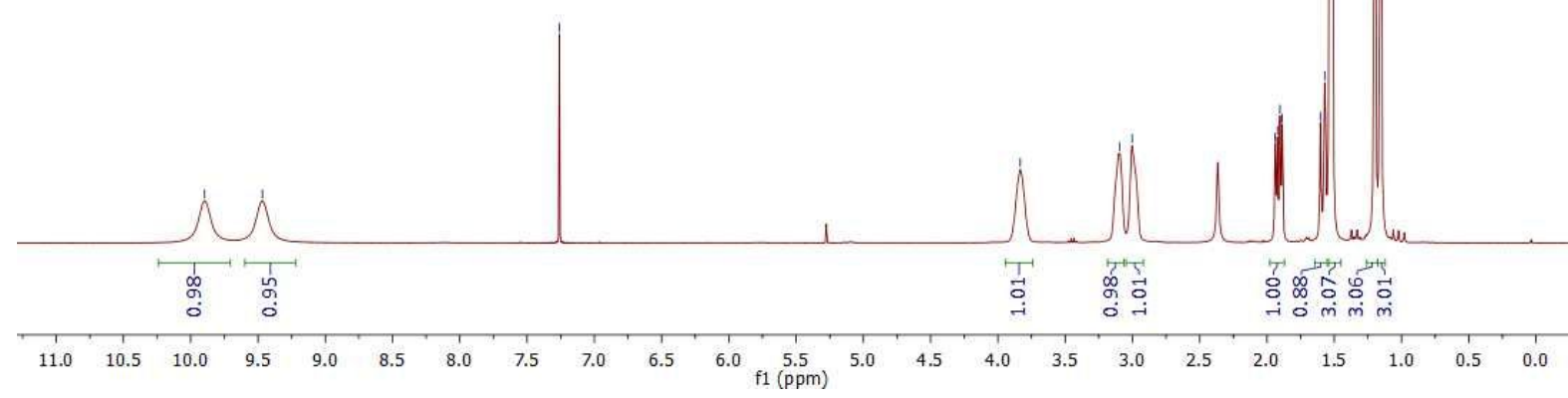

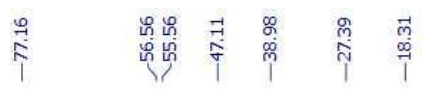

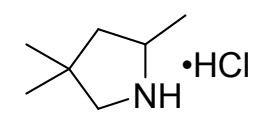

6c

$91 \mathrm{MHz}, \mathrm{CDCl}_{3}$

$\begin{array}{llllllllllllllllllllllllll}210 & 200 & 190 & 180 & 170 & 160 & 150 & 140 & 130 & 120 & 110 & 100 & 90 & 80 & 70 & 60 & 50 & 40 & 30 & 20 & 10 & 0 & -10\end{array}$ 


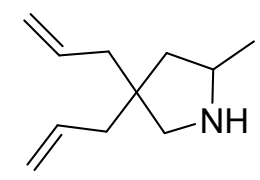

$6 d$

$360 \mathrm{MHz}, \mathrm{CDCl}_{3}$

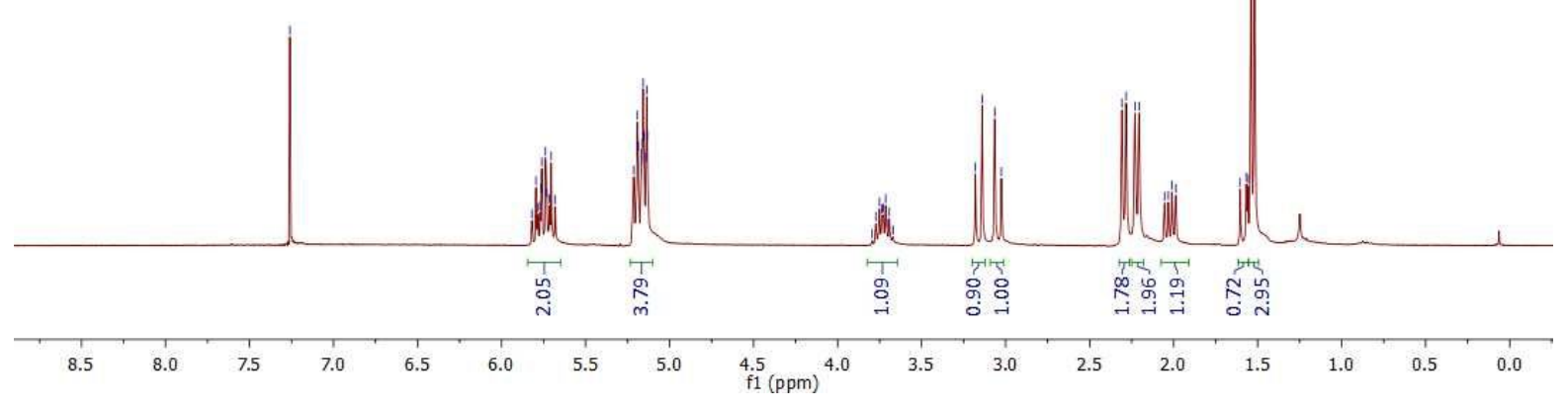

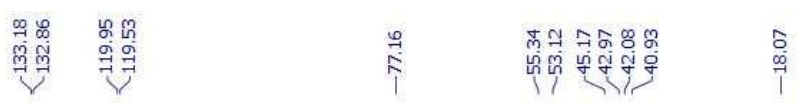

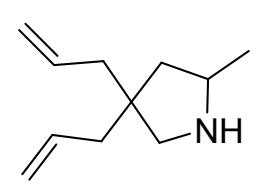

$6 d$

$91 \mathrm{MHz}, \mathrm{CDCl}_{3}$

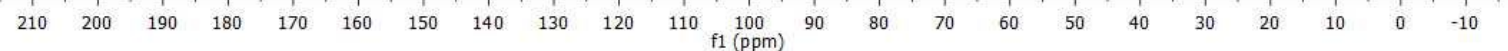




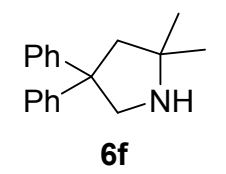

$360 \mathrm{MHz}, \mathrm{CDCl}_{3}$
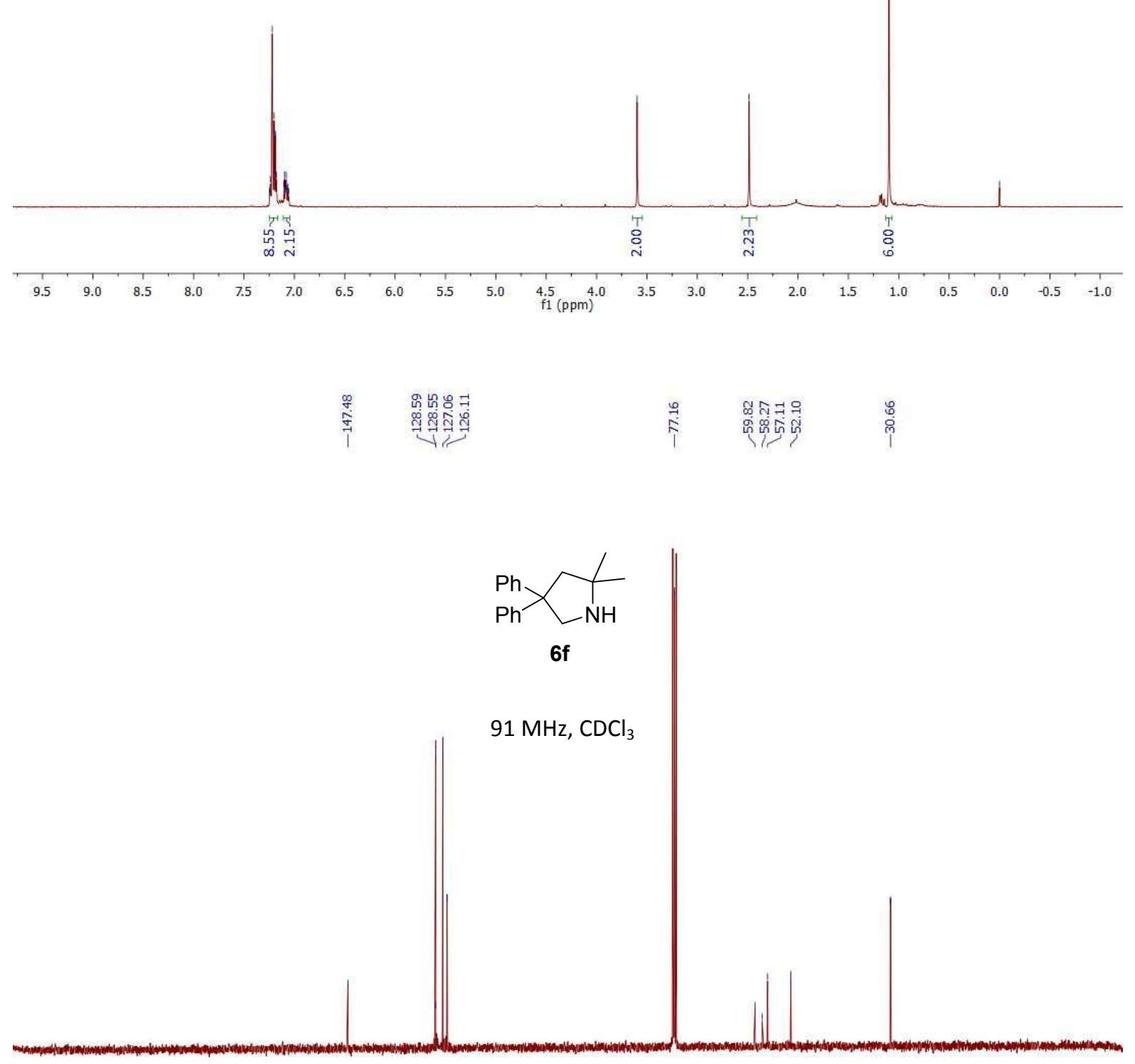

$\begin{array}{llllllllllllllllllllllllllllllllllll}210 & 200 & 190 & 180 & 170 & 160 & 150 & 140 & 130 & 120 & 110 & 100 & 90 & 80 & 70 & 60 & 50 & 40 & 30 & 20 & 10 & 0 & -10\end{array}$ 


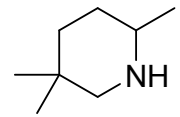

$6 k$

$300 \mathrm{MHz}, \mathrm{CDCl}_{3}$
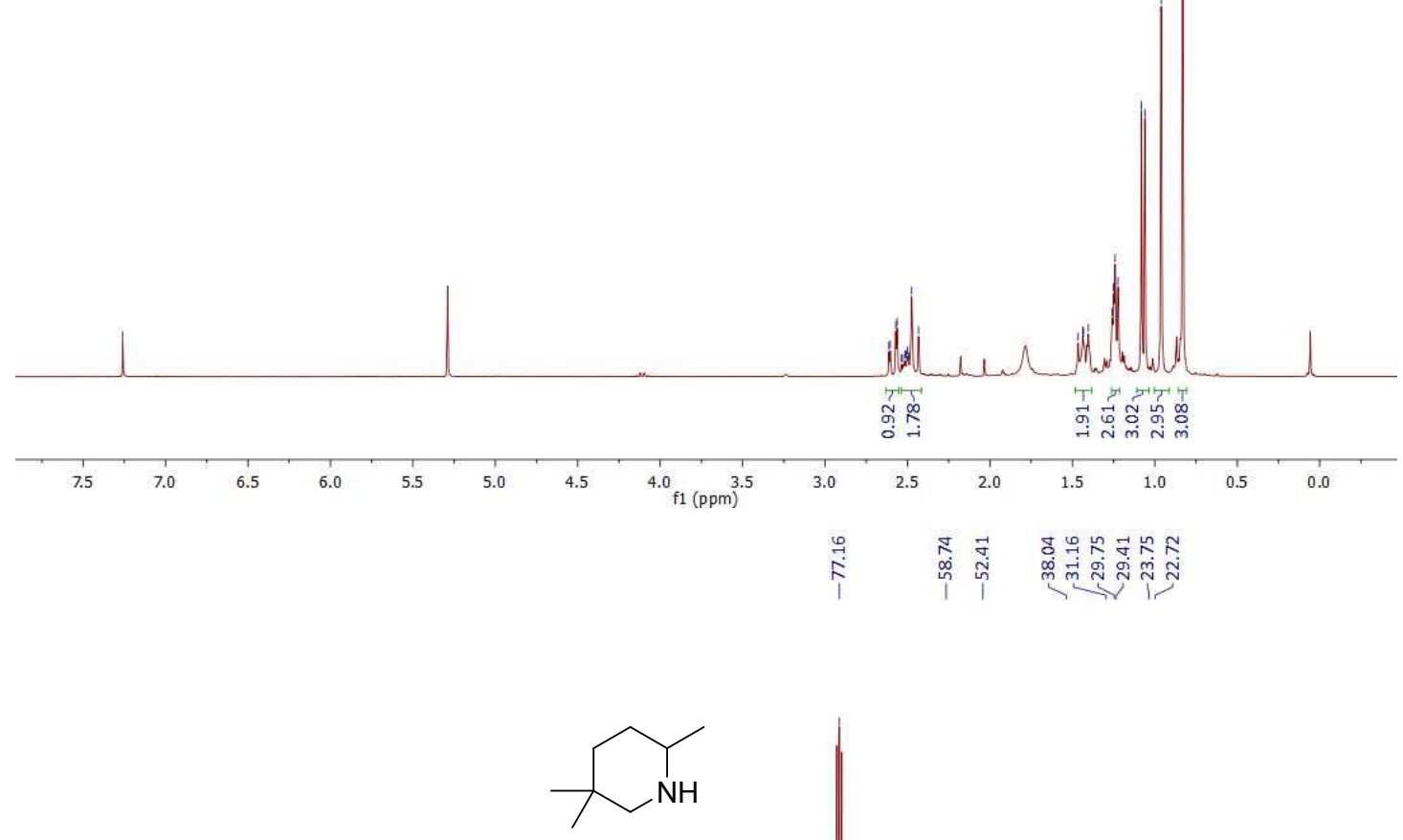

6k

$75 \mathrm{MHz}, \mathrm{CDCl}_{3}$

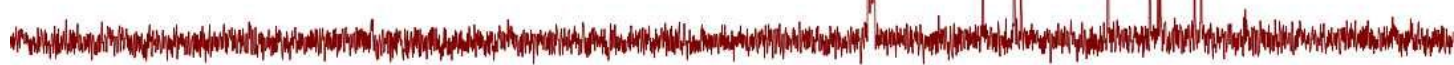

$\begin{array}{lllllllllllllllllllllll}210 & 200 & 190 & 180 & 170 & 160 & 150 & 140 & 130 & 120 & 110 & 100 & 90 & 80 & 70 & 60 & 50 & 40 & 30 & 20 & 10 & 0 & -10\end{array}$ 


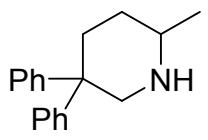

6I

$360 \mathrm{MHz}, \mathrm{CDCl}_{3}$

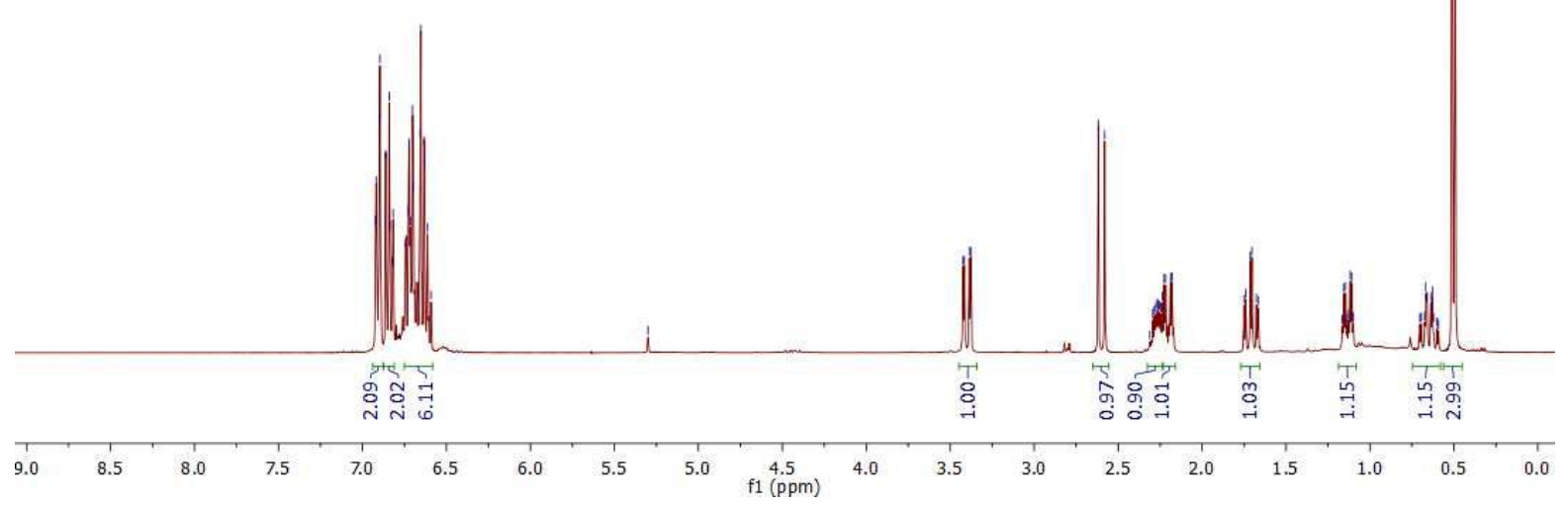

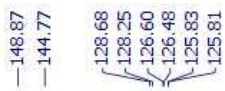

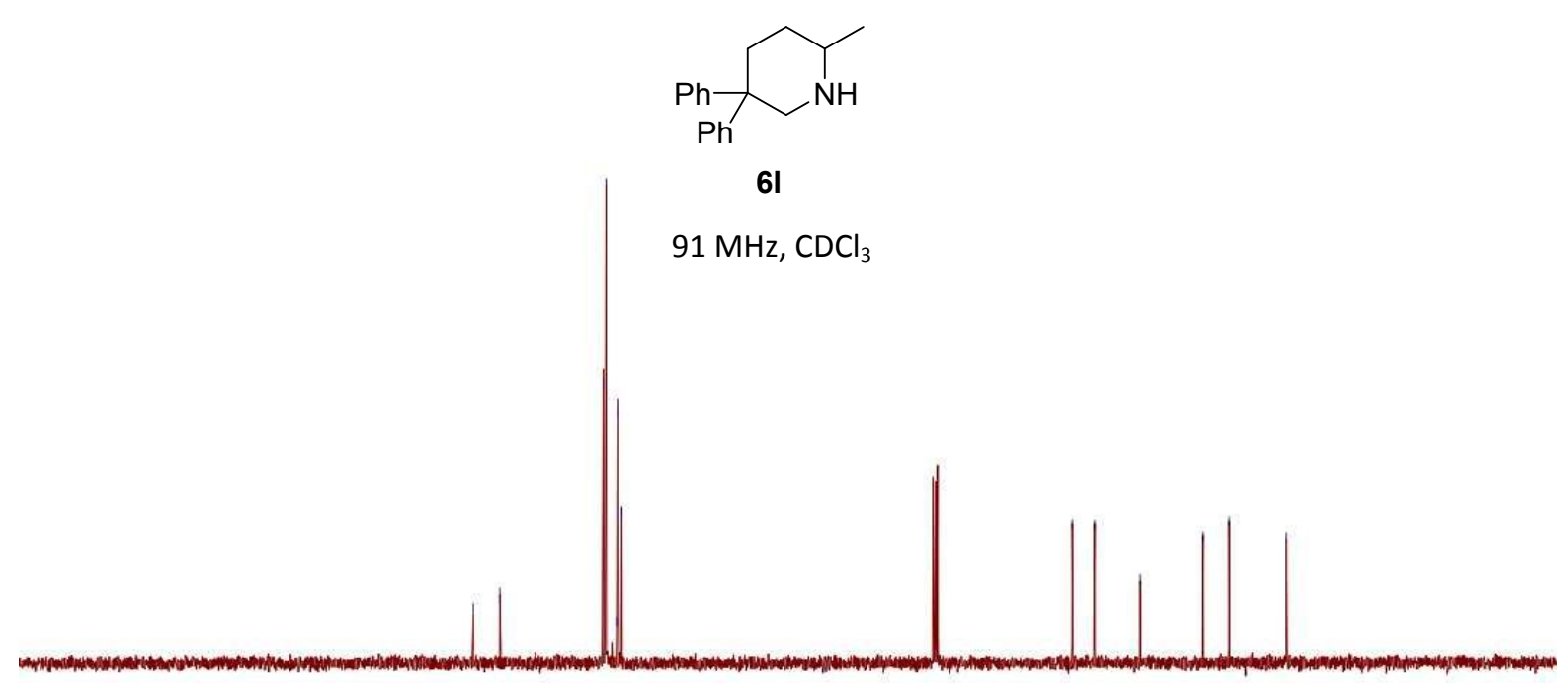

$\begin{array}{lllll}210 & 200 & 190 & 180 & 1\end{array}$

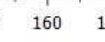

$50 \quad 140$

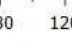

$110 \underset{\mathrm{f} 1}{100}(\mathrm{ppm})$

80

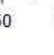




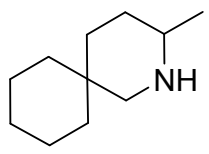

$6 m$

$300 \mathrm{MHz}, \mathrm{CDCl}_{3}$

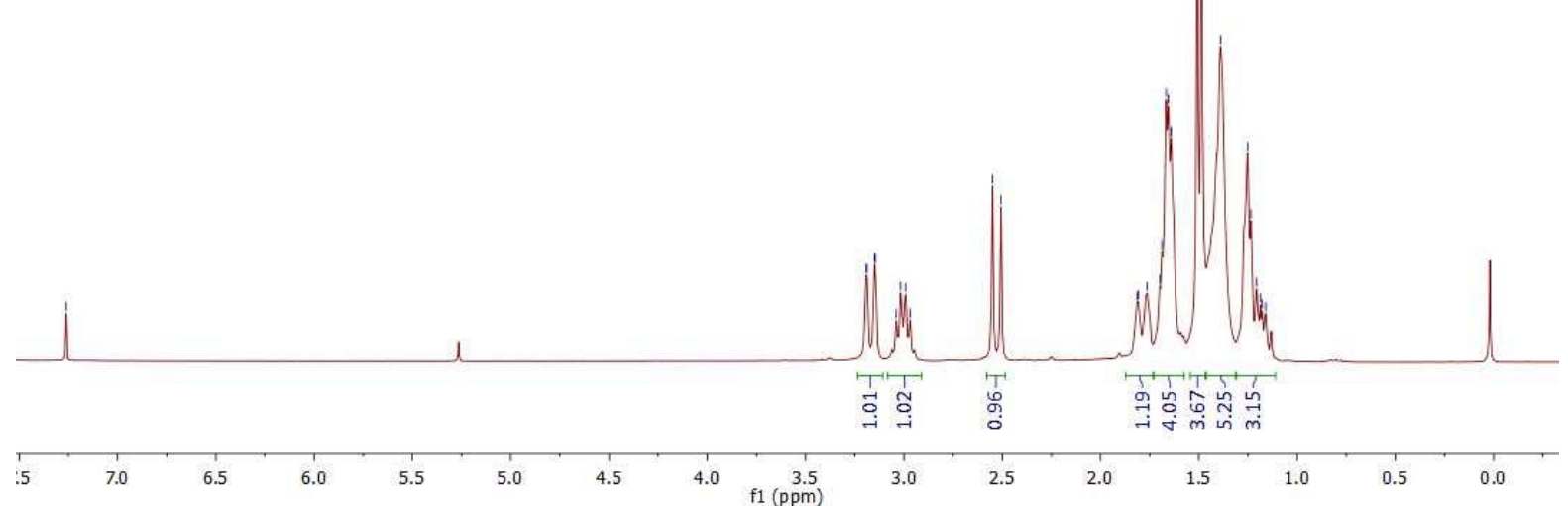

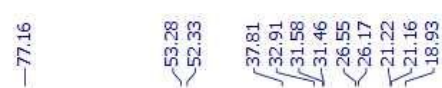

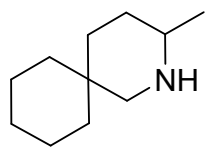

$6 m$

$75 \mathrm{MHz}, \mathrm{CDCl}_{3}$

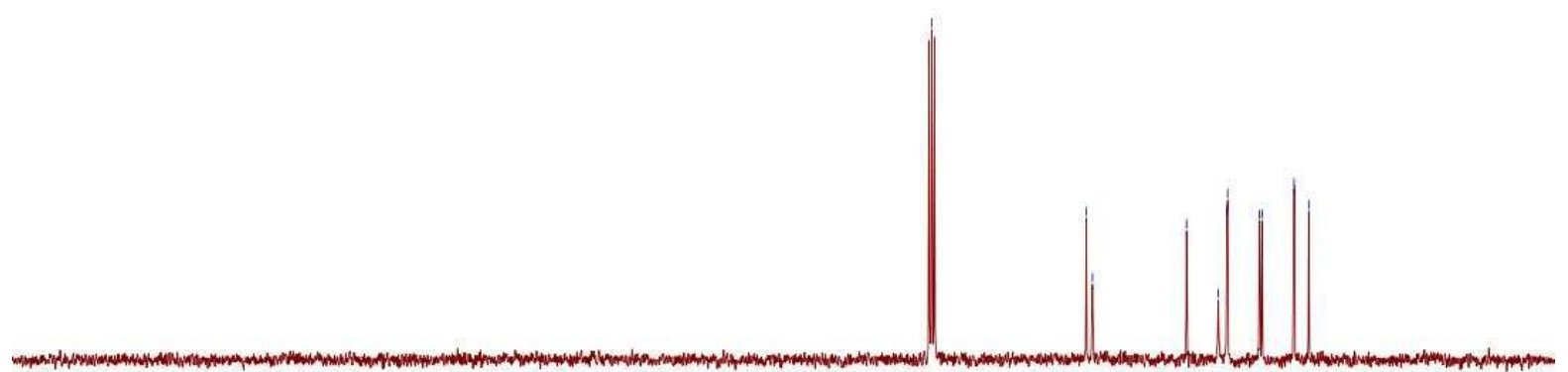

$\begin{array}{llllllllllllllllllllllllllllllll}210 & 200 & 190 & 180 & 170 & 160 & 150 & 140 & 130 & 120 & 110 & 100 & 90 & 80 & 70 & 60 & 50 & 40 & 30 & 20 & 10 & 0 & -10\end{array}$ 


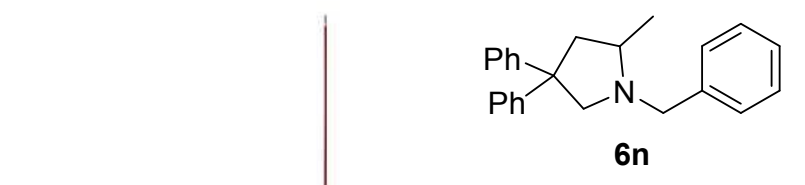

$250 \mathrm{MHz}, \mathrm{CDCl}_{3}$

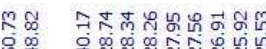

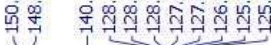

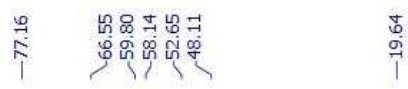

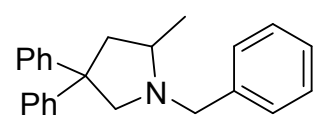

6n

$91 \mathrm{MHz}, \mathrm{CDCl}_{3}$

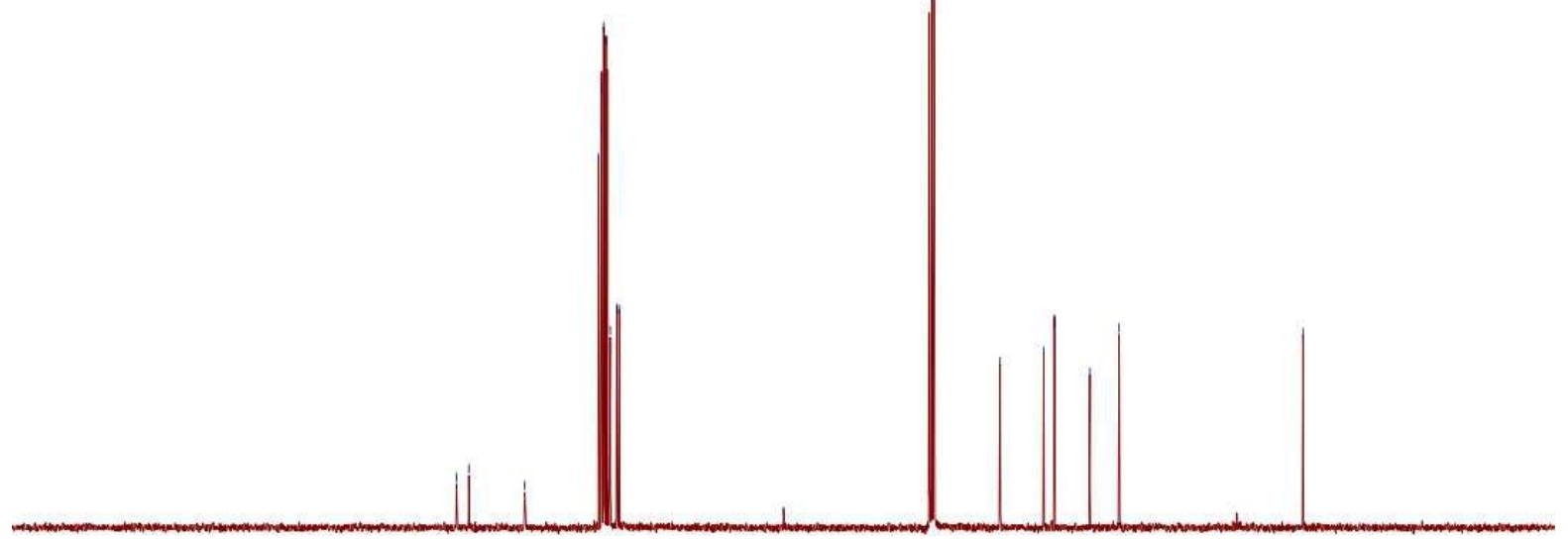

$\begin{array}{llllllllllllllllllllllllllllllll}210 & 200 & 190 & 180 & 170 & 160 & 150 & 140 & 130 & 120 & 110 & 100 & 90 & 80 & 70 & 60 & 50 & 40 & 30 & 20 & 10 & 0 & -10\end{array}$ 

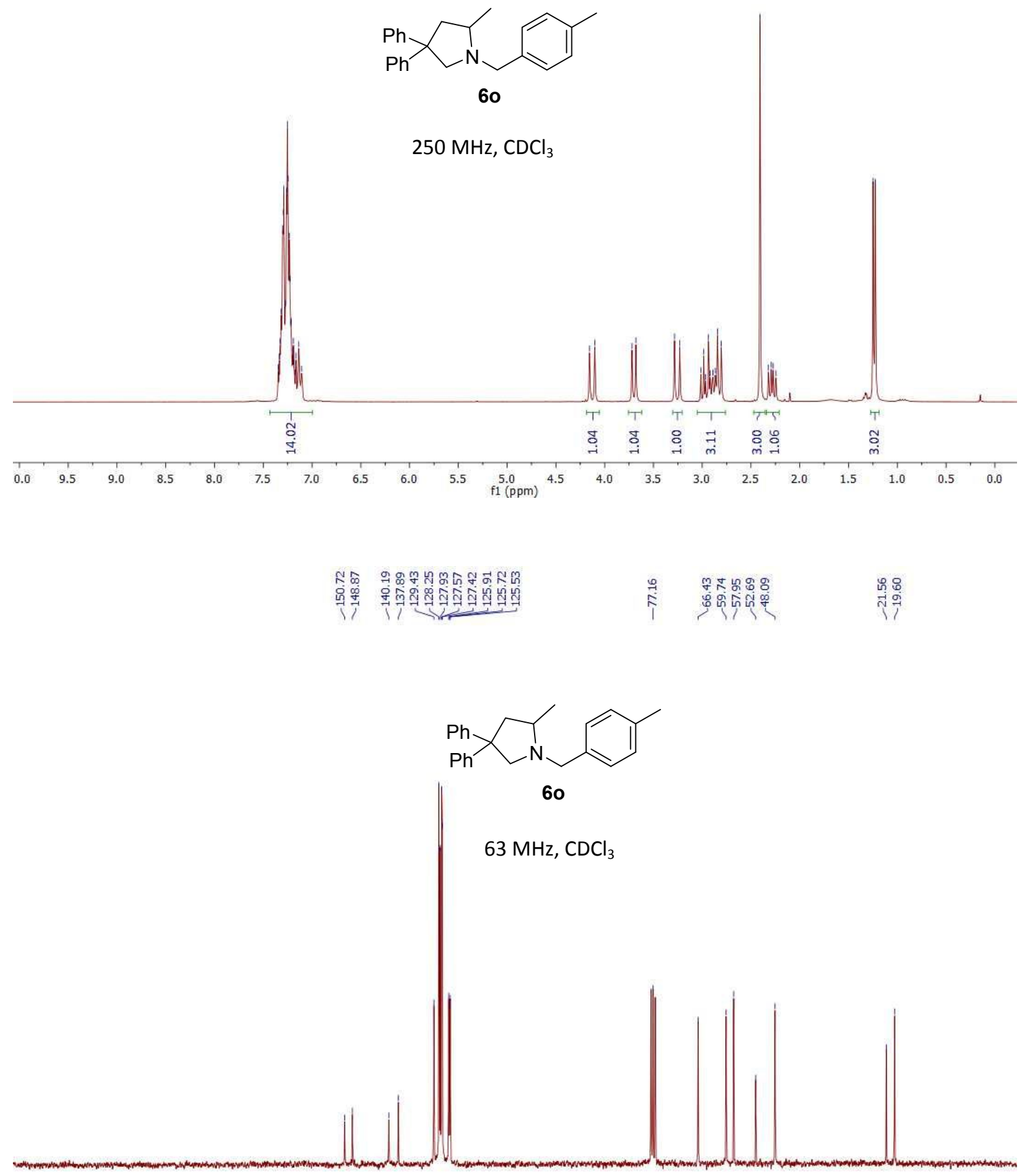

$\begin{array}{lllllllllllllllllllllll}220 & 210 & 200 & 190 & 180 & 170 & 160 & 150 & 140 & 130 & 120 & \begin{array}{l}110 \\ \mathrm{f} 1(\mathrm{ppm})\end{array} & 100 & 90 & 80 & 70 & 60 & 50 & 40 & 30 & 20 & 10 & 0\end{array}$ 


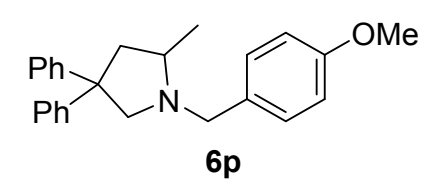

$250 \mathrm{MHz}, \mathrm{CDCl}_{3}$
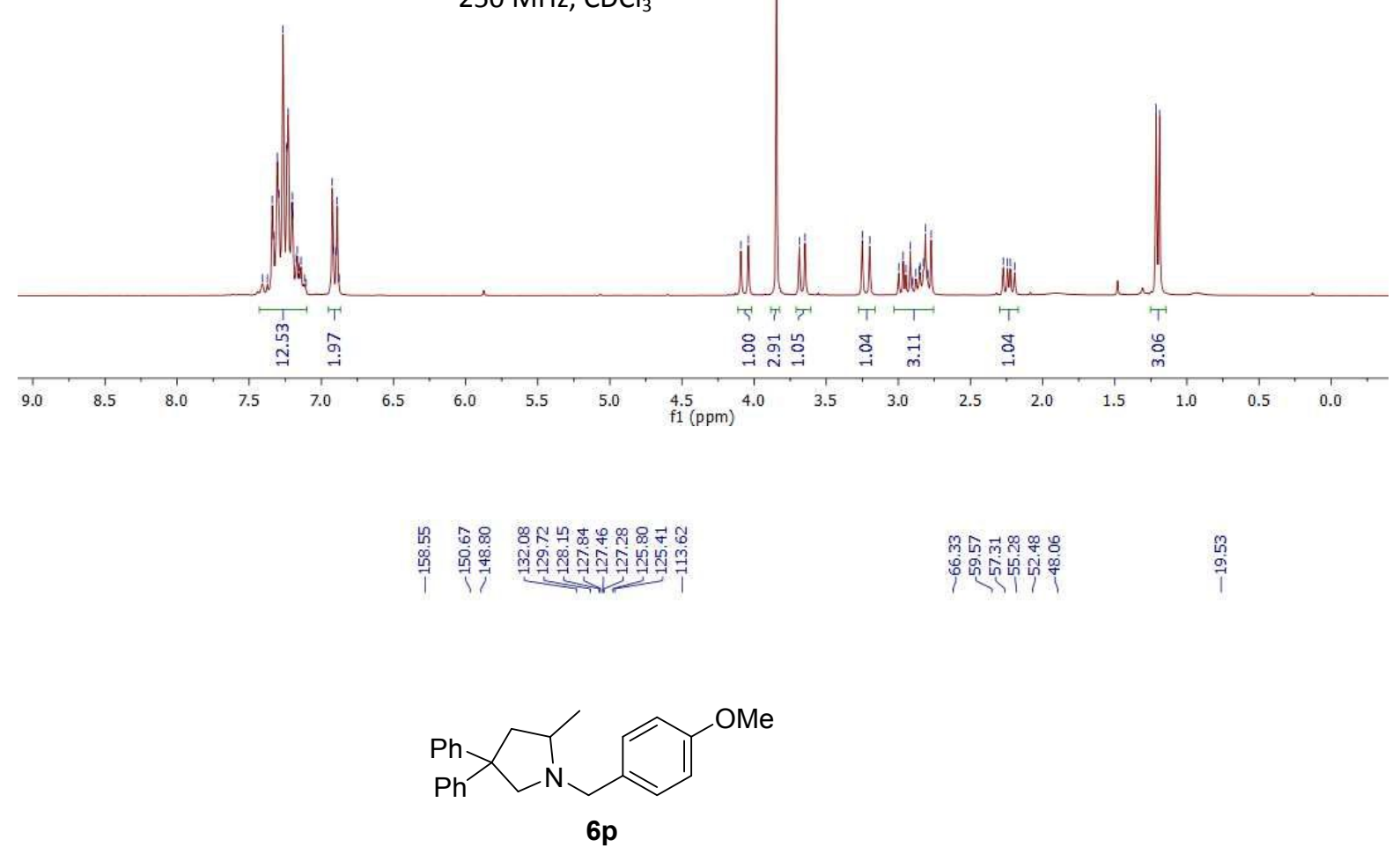

$63 \mathrm{MHz}, \mathrm{CDCl}_{3}$

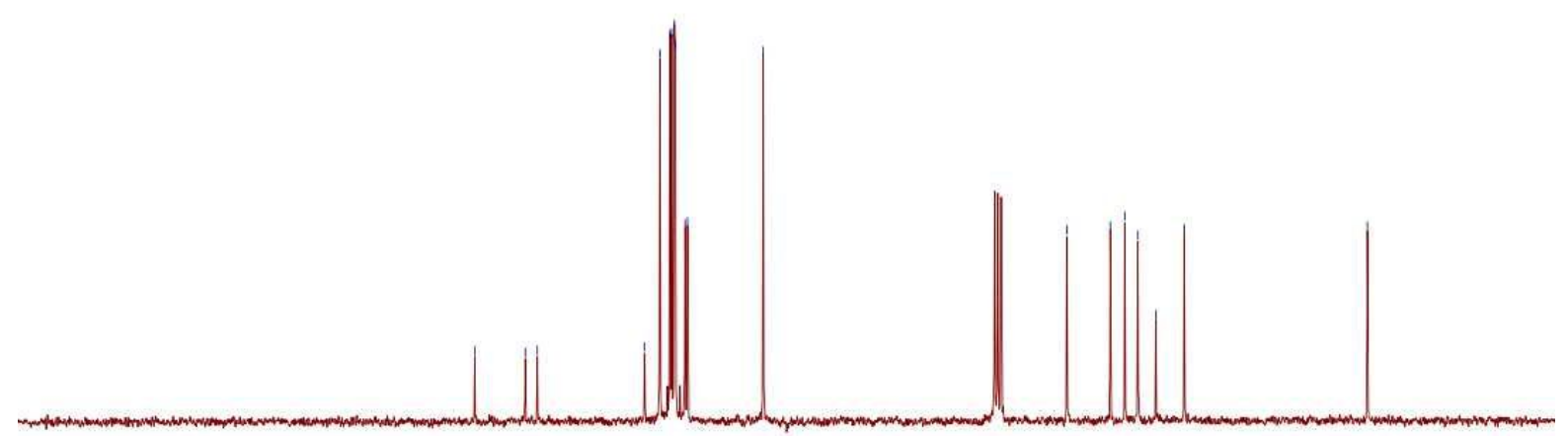

$\begin{array}{llllllllllllllllllllllll}220 & 210 & 200 & 190 & 180 & 170 & 160 & 150 & 140 & 130 & 120 & 110 & 100 & 90 & 80 & 70 & 60 & 50 & 40 & 30 & 20 & 10\end{array}$ 


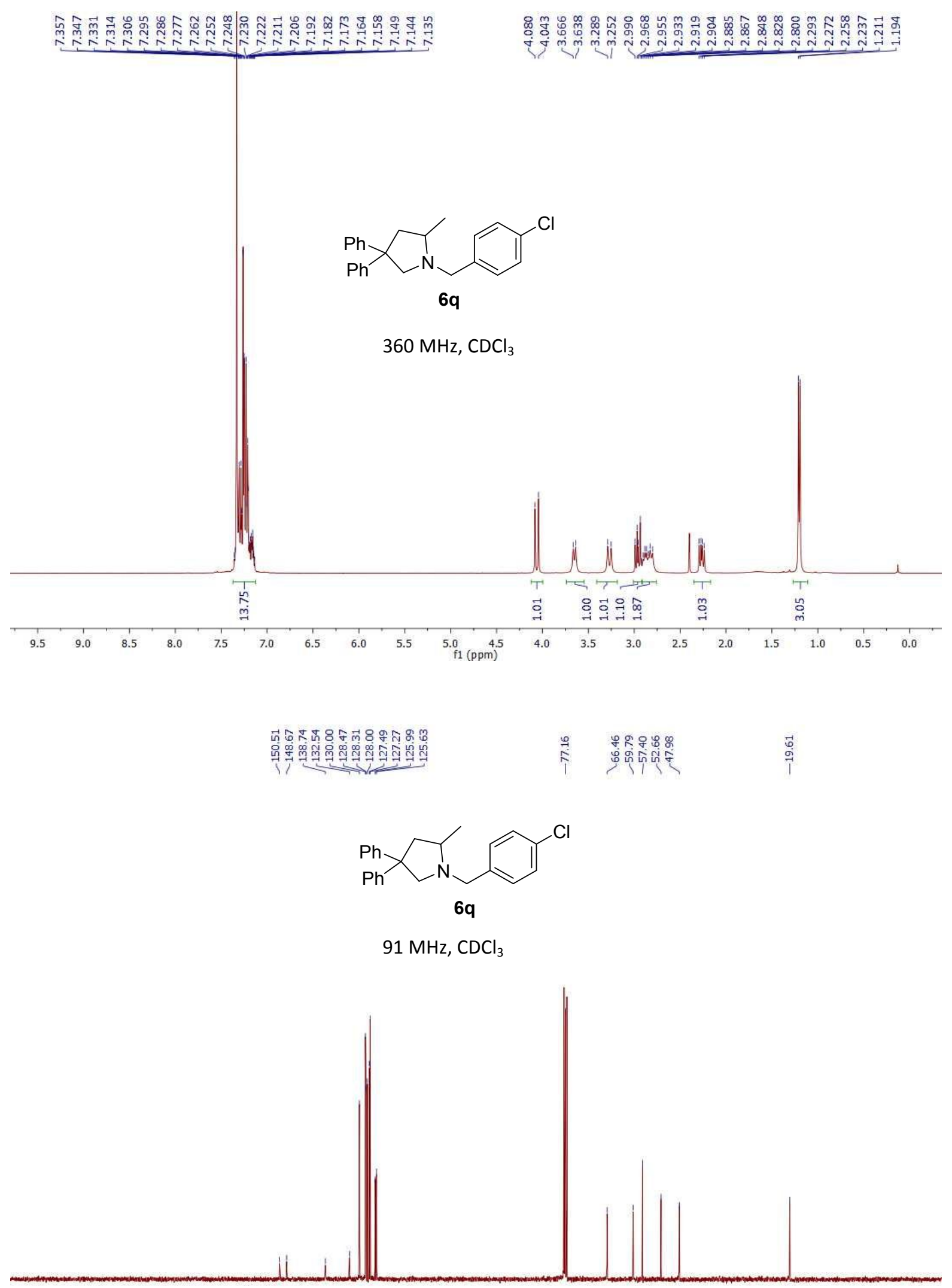

$\begin{array}{lllllllllllllllllllllllllllllllllll}210 & 200 & 190 & 180 & 170 & 160 & 150 & 140 & 130 & 120 & 110 & 100 & 90 & 80 & 70 & 60 & 50 & 40 & 30 & 20 & 10 & 0 & -10\end{array}$ 


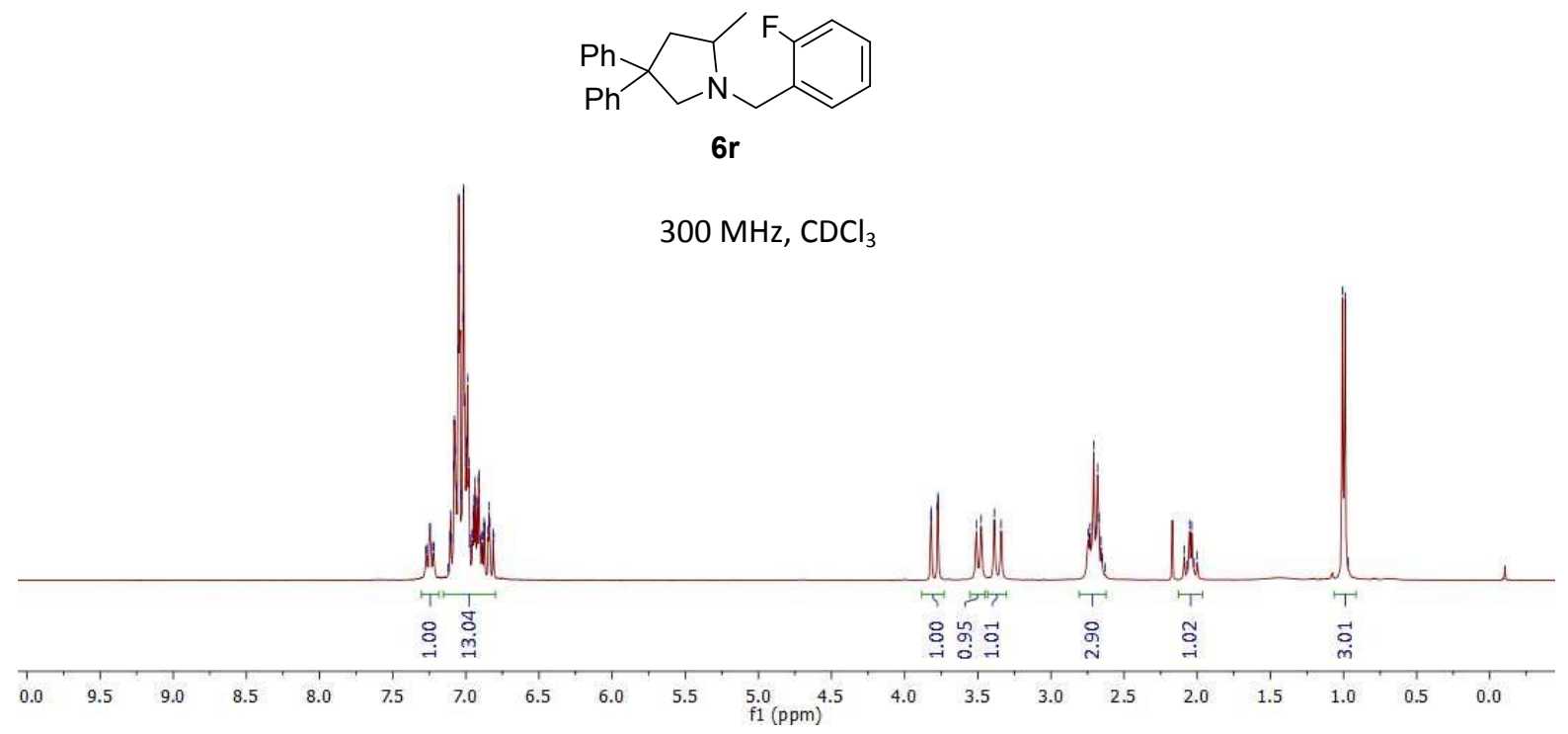

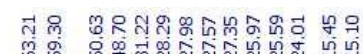

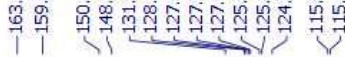

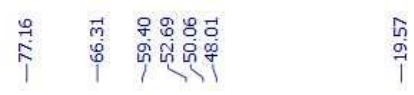

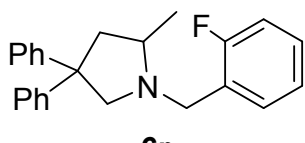

$6 r$

$63 \mathrm{MHz}^{\mathrm{CDCl}} \mathrm{CD}_{3}$

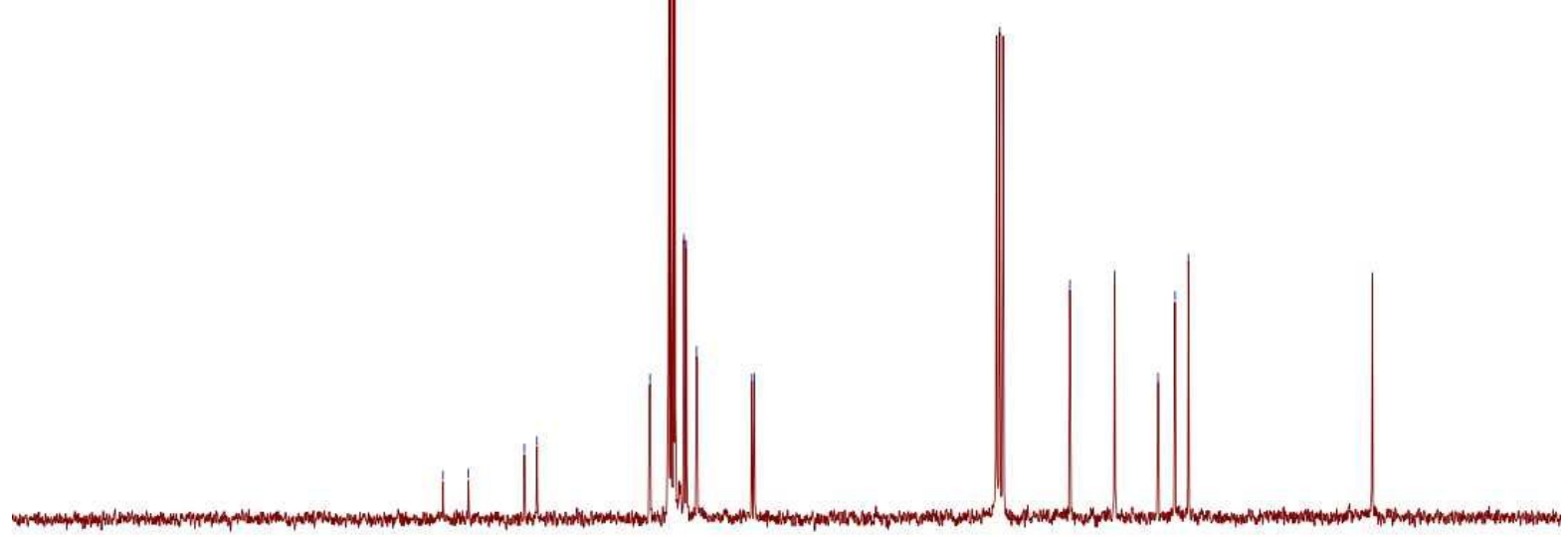

$\begin{array}{lllllllllllllllllllllll}220 & 210 & 200 & 190 & 180 & 170 & 160 & 150 & 140 & 130 & 120 & \begin{array}{c}110 \\ \mathrm{f} 1(\mathrm{ppm})\end{array} & 100 & 90 & 80 & 70 & 60 & 50 & 40 & 30 & 20 & 10 & 0\end{array}$ 


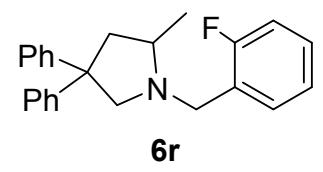

$235 \mathrm{MHz}, \mathrm{CDCl}_{3}$

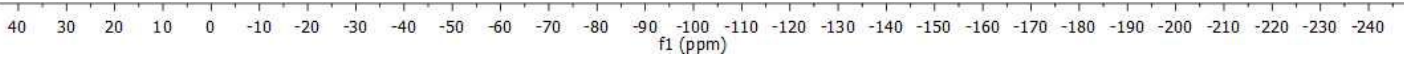




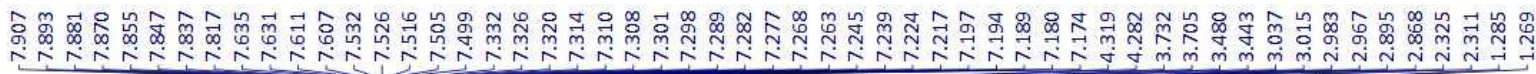

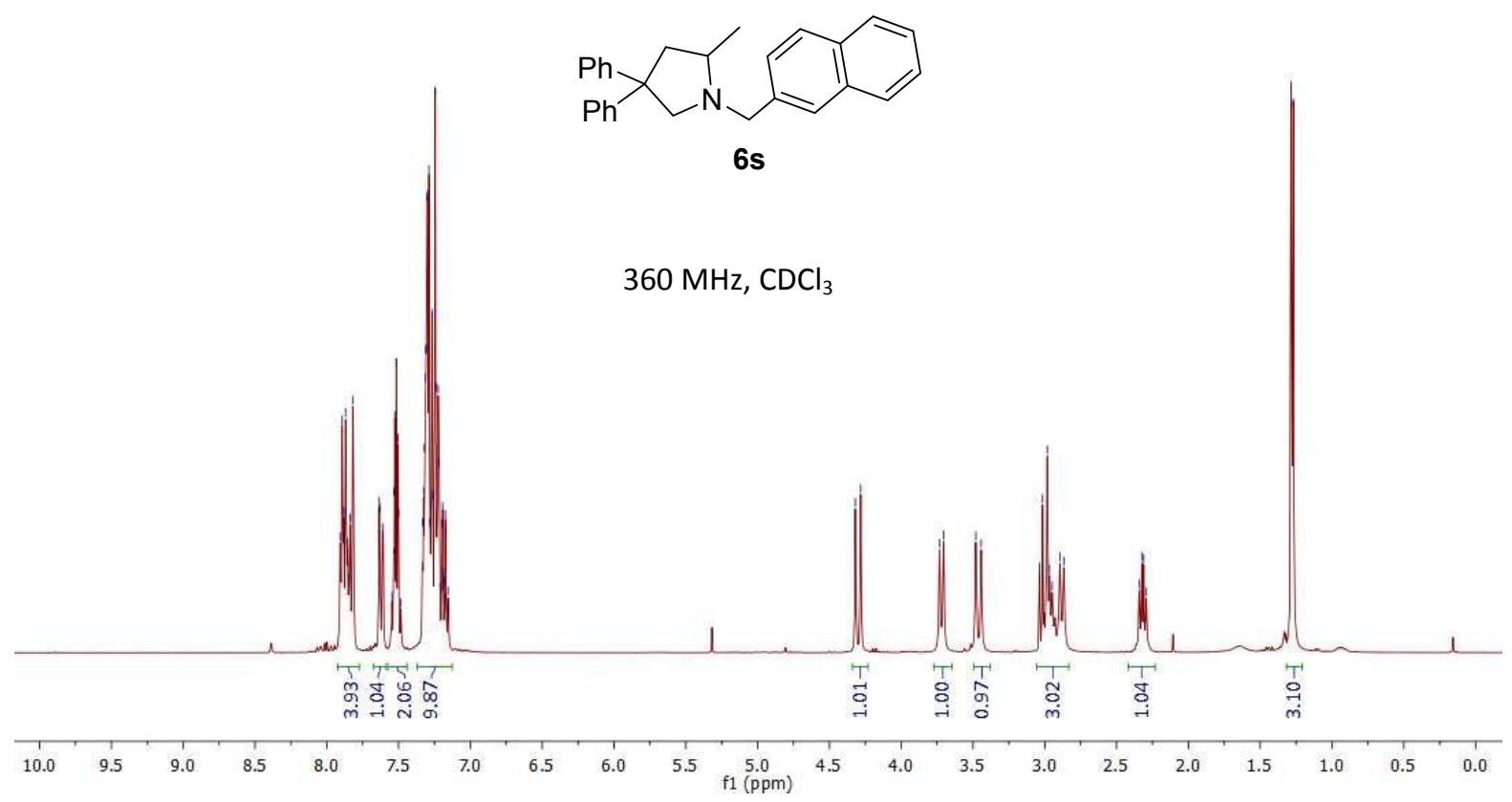

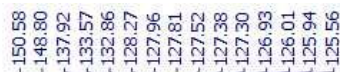

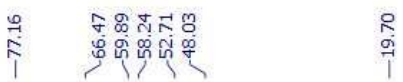

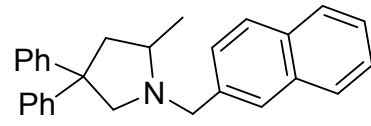

6s

$63 \mathrm{MHz}, \mathrm{CDCl}_{3}$

$\begin{array}{llllllllllllllllllllllll}220 & 210 & 200 & 190 & 180 & 170 & 160 & 150 & 140 & 130 & 120 & \begin{array}{l}110 \\ \mathrm{f} 1(\mathrm{ppm})\end{array} & 100 & 90 & 80 & 70 & 60 & 50 & 40 & 30 & 20 & 10 & 0\end{array}$ 

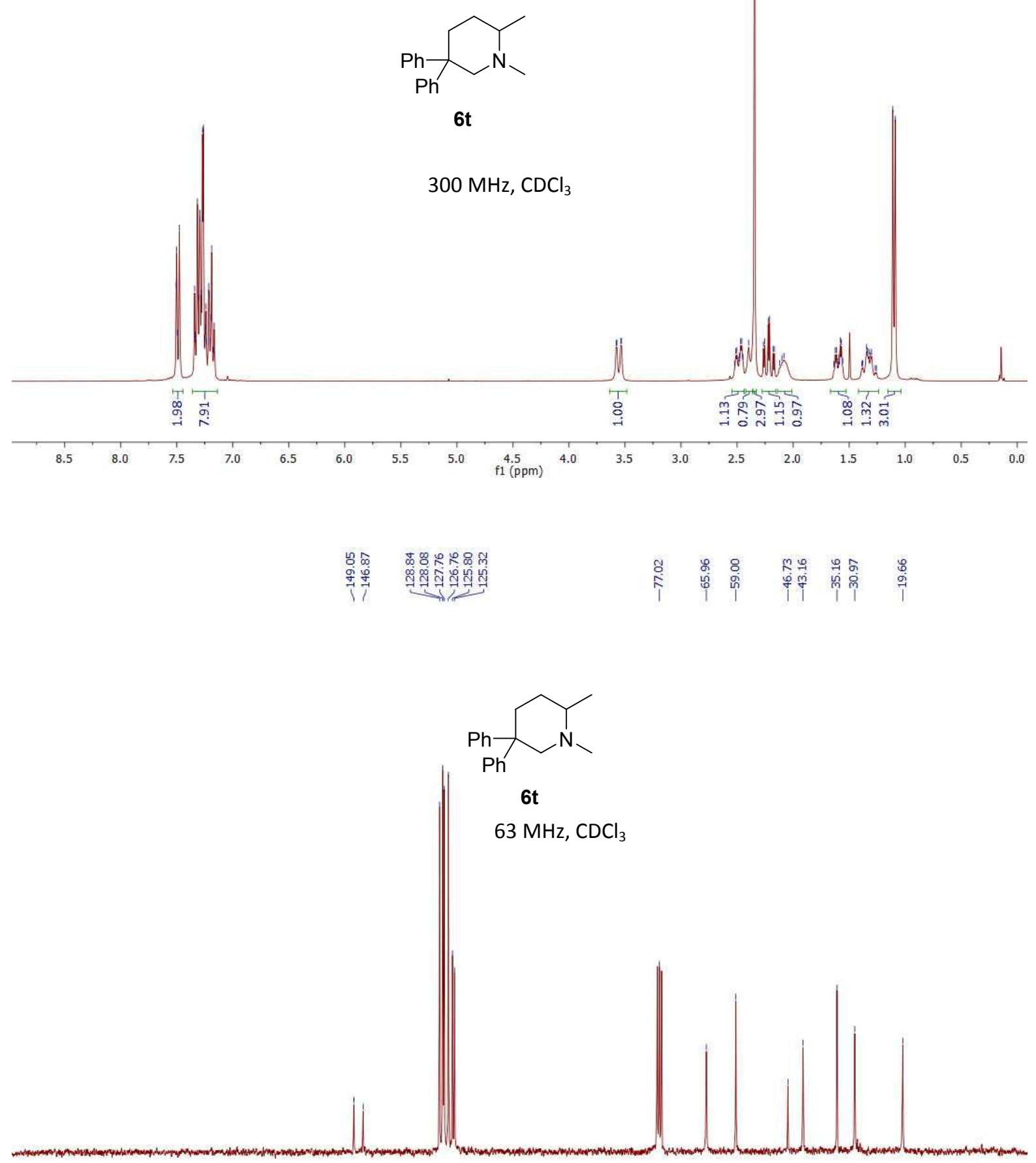

$\begin{array}{llllllllllllllllllllllllllll}220 & 210 & 200 & 190 & 180 & 170 & 160 & 150 & 140 & 130 & 120 & 110 & 100 & 90 & 80 & 70 & 60 & 50 & 40 & 30 & 20 & 10 & 0\end{array}$ 


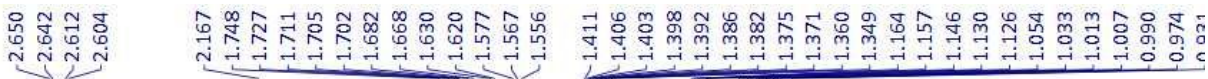

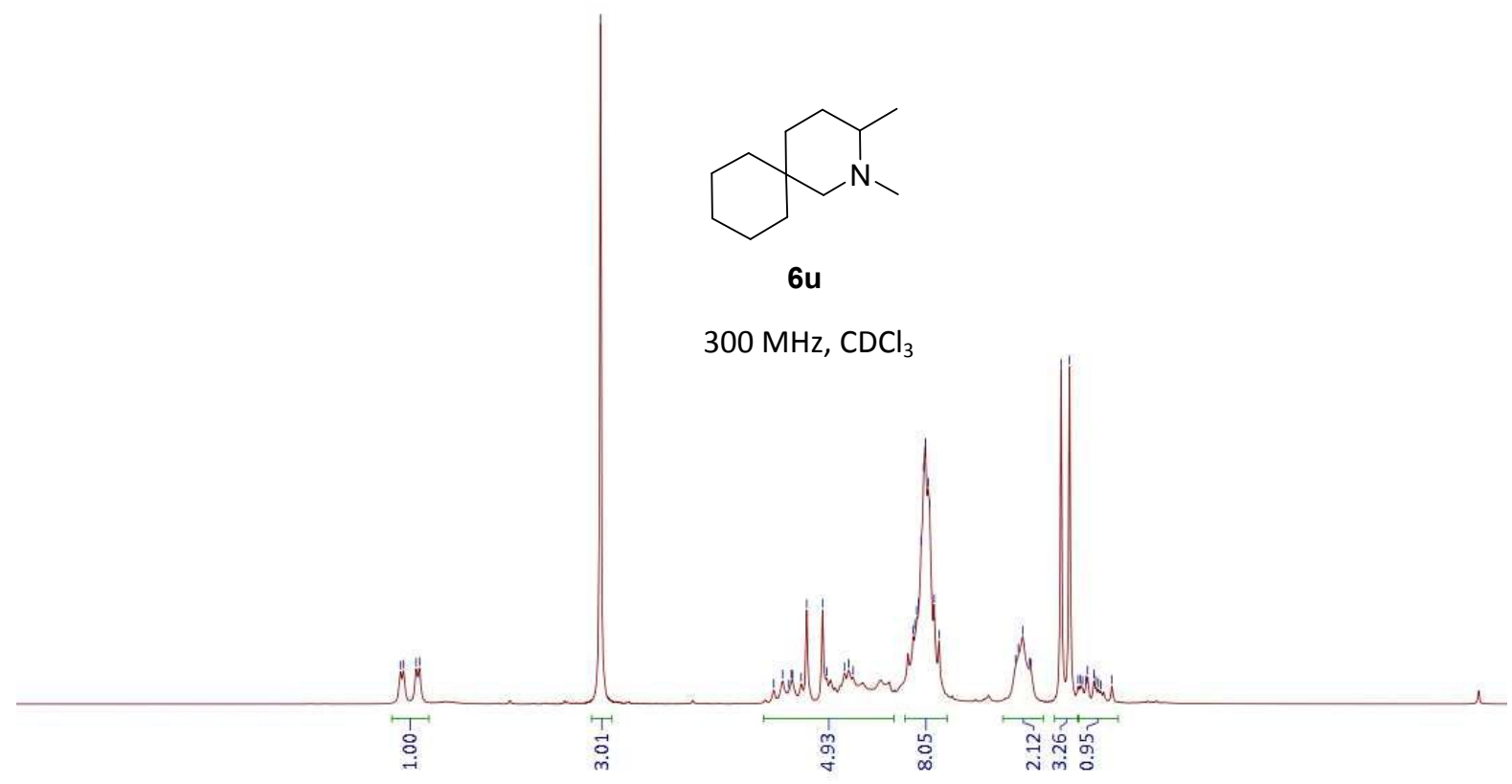

$\begin{array}{lllllllllllllllllllllllllllllllllllllllllllllll}3.5 & 3.4 & 3.3 & 3.2 & 3.1 & 3.0 & 2.9 & 2.8 & 2.7 & 2.6 & 2.5 & 2.4 & 2.3 & 2.2 & 2.1 & 2.0 & 1.9 & 1.8 & 1.7 & 1.6 & 1.5 & 1.4 & 1.3 & 1.2 & 1.1 & 1.0 & 0.9 & 0.8 & 0.7 & 0.6 & 0.5 & 0.4 & 0.3 & 0.2 & 0.1 & 0.0\end{array}$
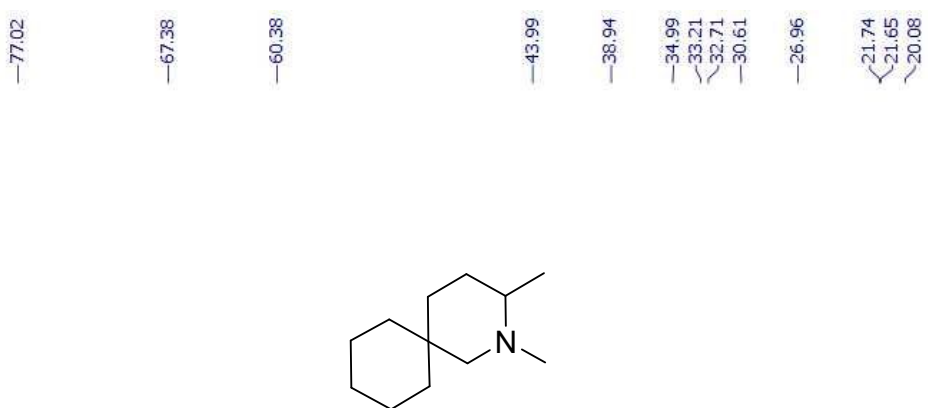

6u

$75 \mathrm{MHz}, \mathrm{CDCl}_{3}$

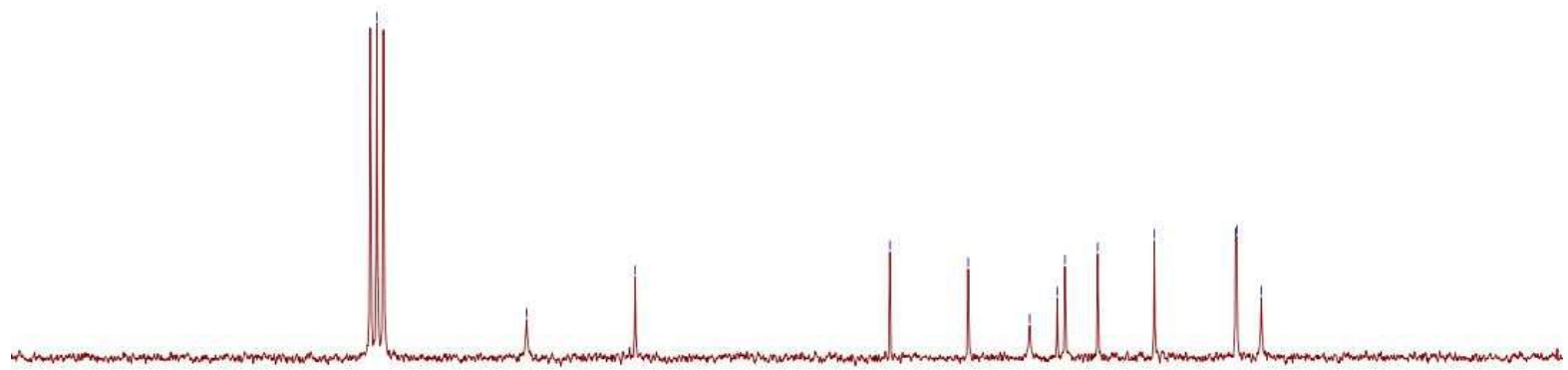

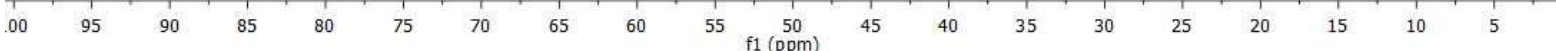



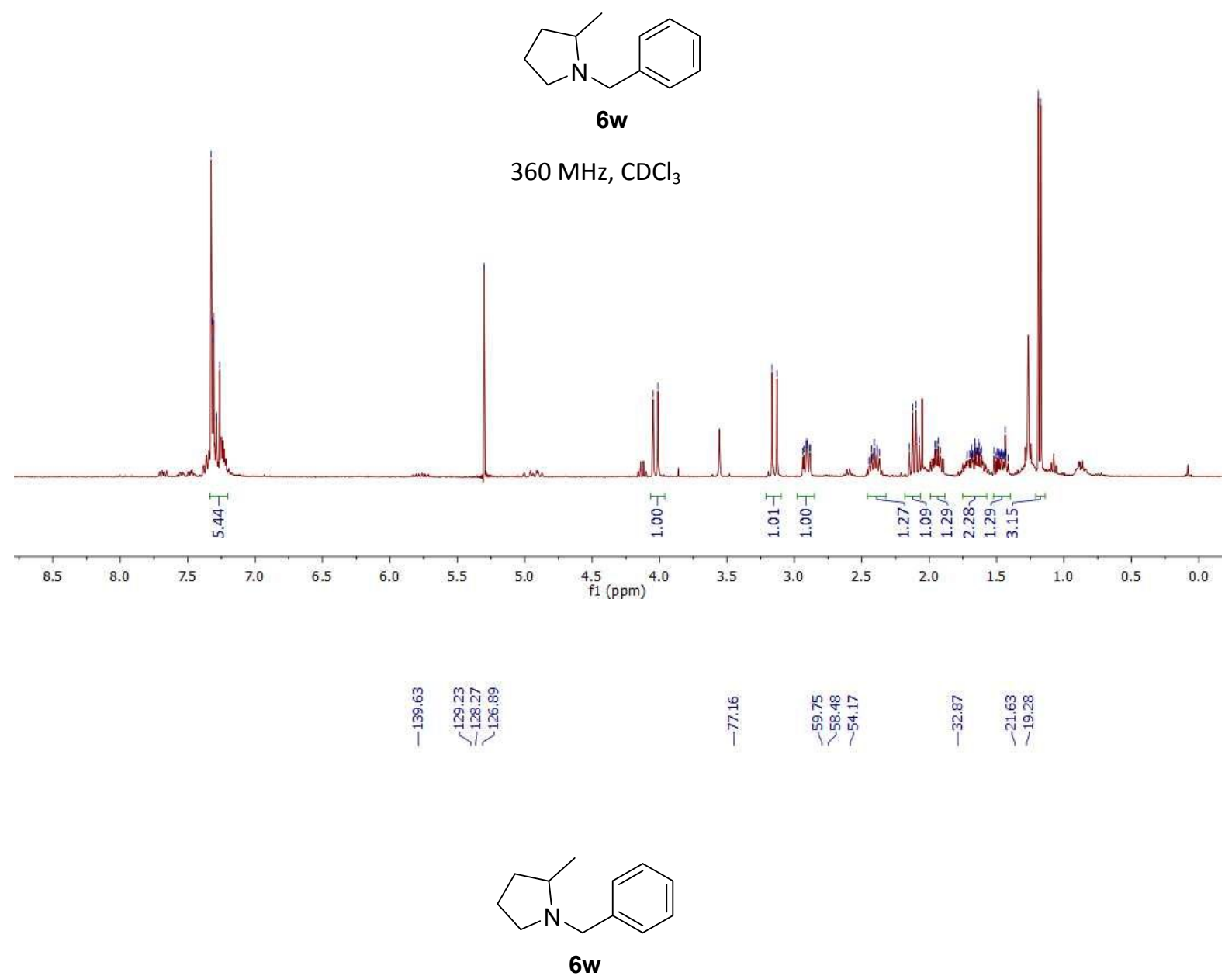

$91 \mathrm{MHz}, \mathrm{CDCl}_{3}$

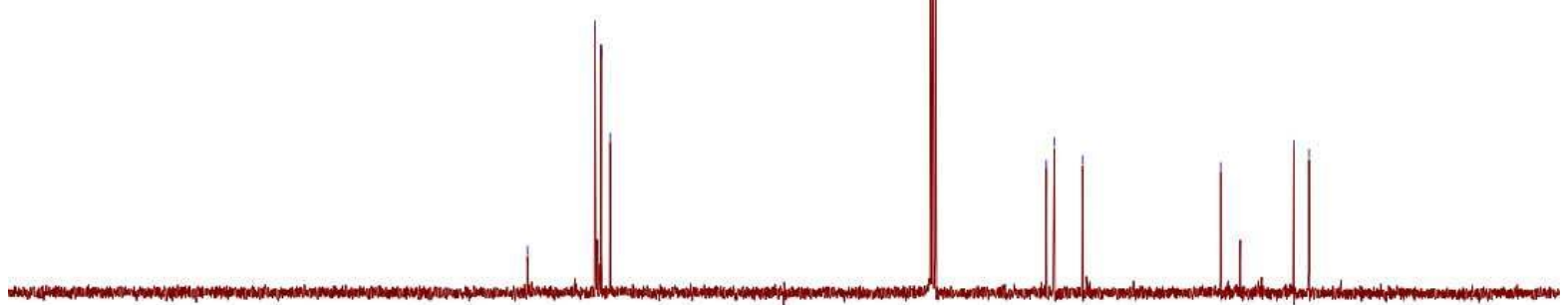

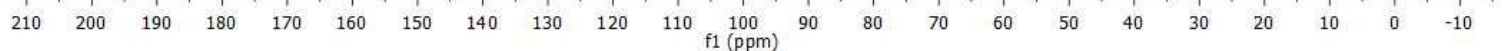



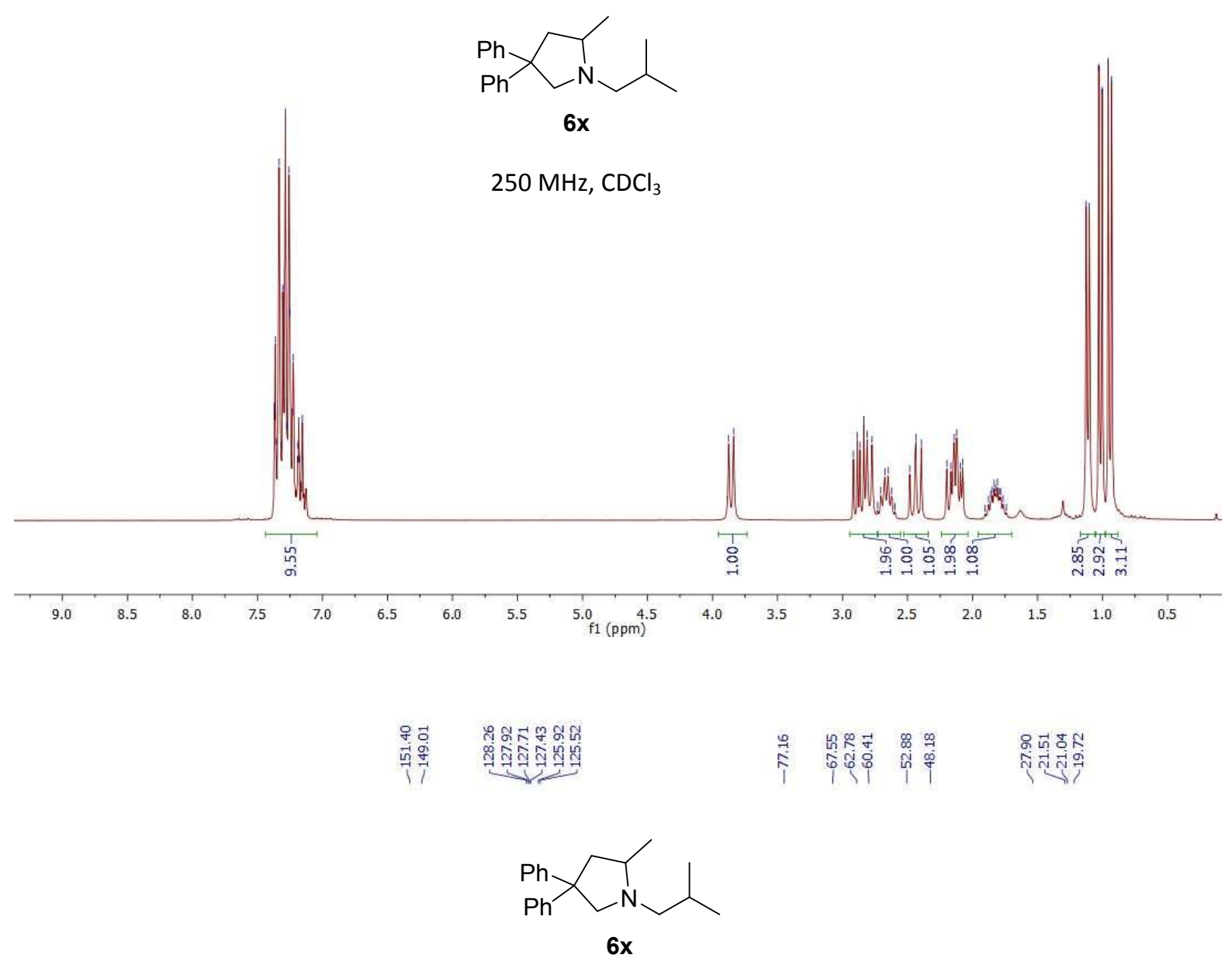

$63 \mathrm{MHz}, \mathrm{CDCl}_{3}$

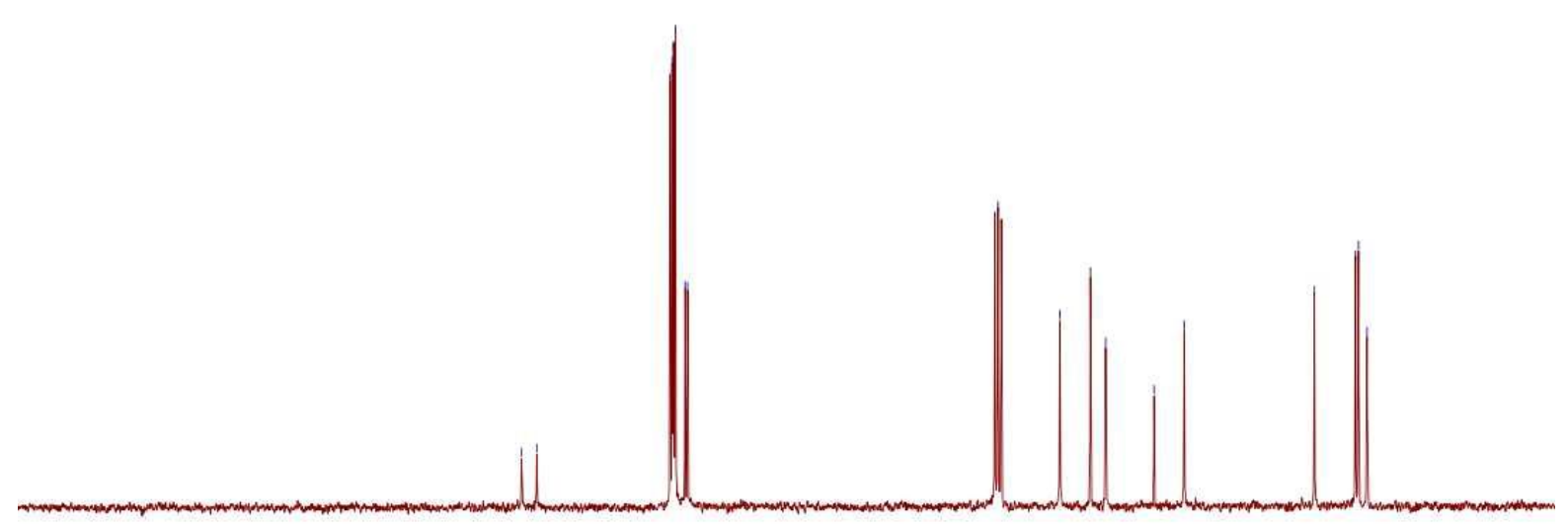

$\begin{array}{lllllllllllllllllllllllllll}220 & 210 & 200 & 190 & 180 & 170 & 160 & 150 & 140 & 130 & 120 & 110 & 100 & 90 & 80 & 70 & 60 & 50 & 40 & 30 & 20 & 10 & 0 & \end{array}$ 


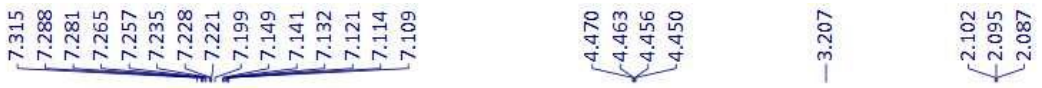

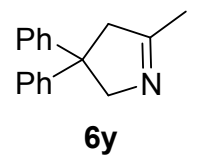

$250 \mathrm{MHz}, \mathrm{CDCl}_{3}$

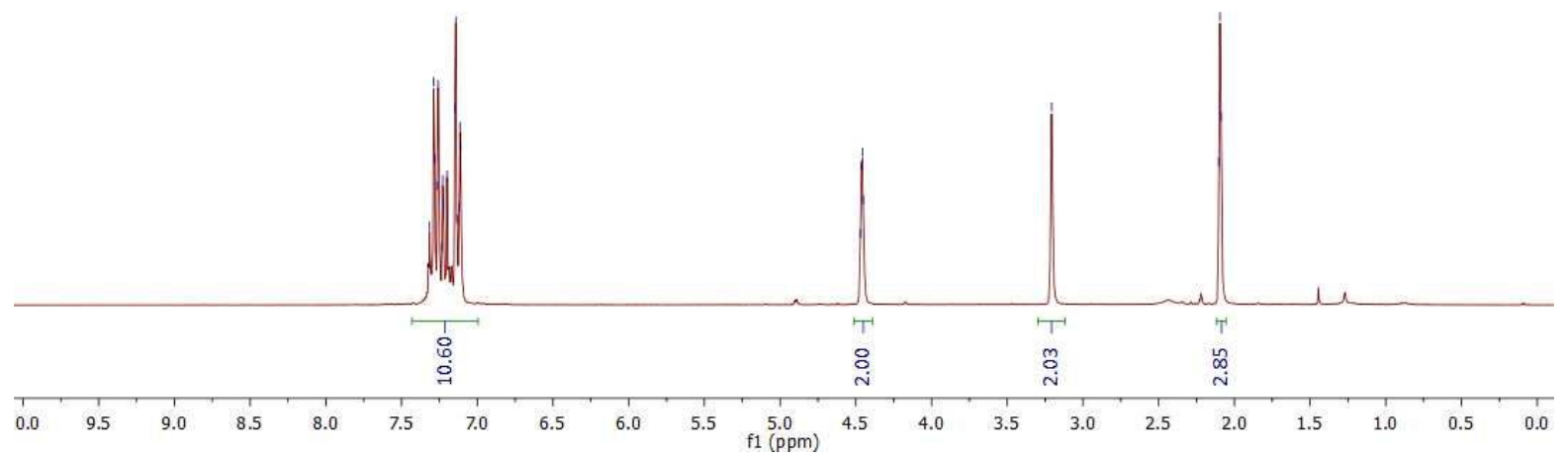

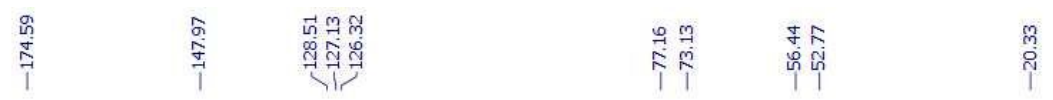

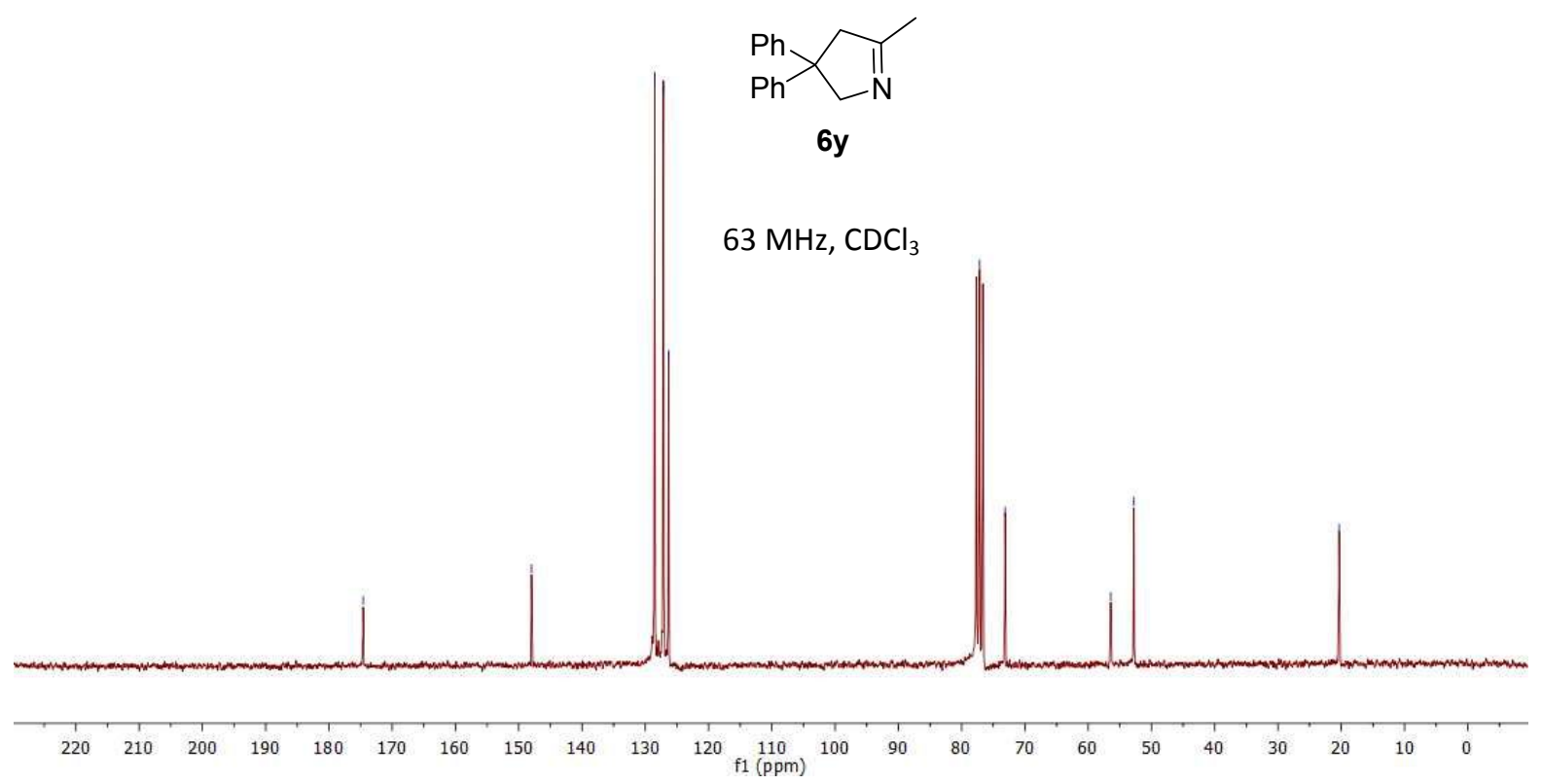



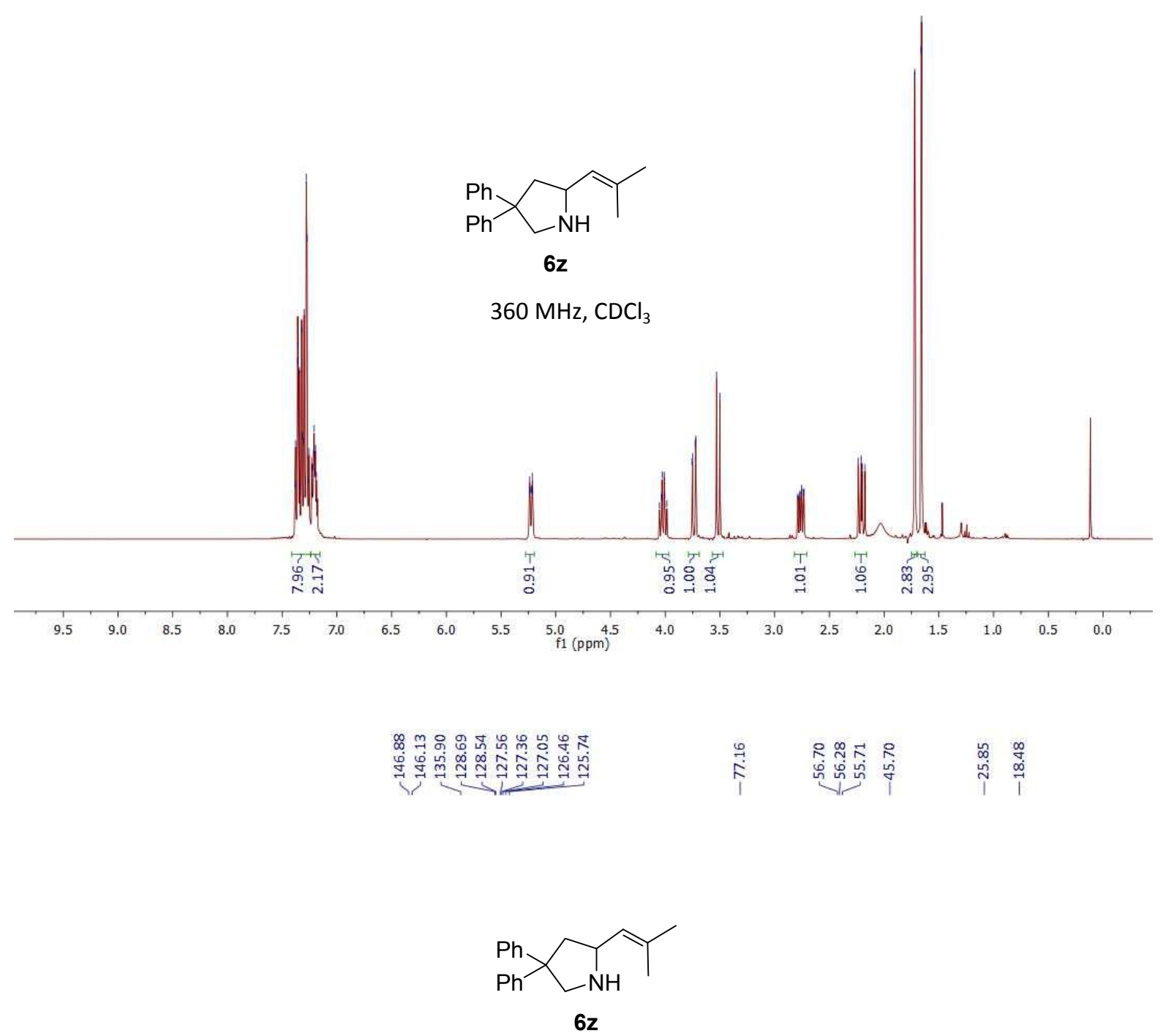

$63 \mathrm{MHz}, \mathrm{CDCl}_{3}$

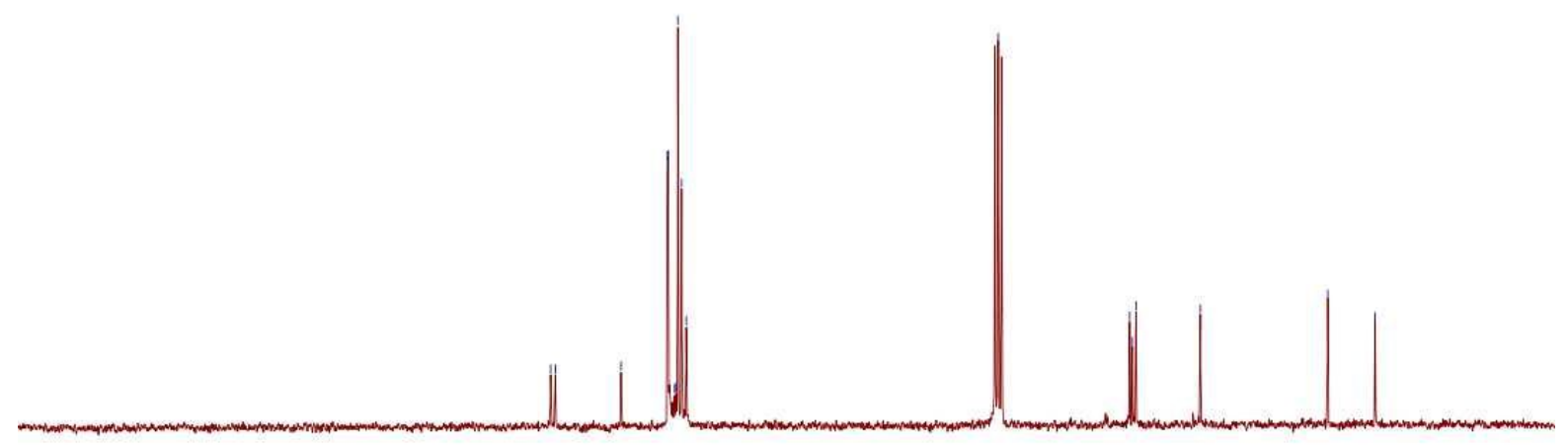

$\begin{array}{lllllllllllllllllllllllll}220 & 210 & 200 & 190 & 180 & 170 & 160 & 150 & 140 & 130 & 120 & 110 & 100 & 90 & 80 & 70 & 60 & 50 & 40 & 30 & 20 & 10 & 0\end{array}$ 